\title{
Evidence-based Clinical Practice Guideline for CKD 2013
}

\author{
Japanese Society of Nephrology
}

Published online: 9 May 2014

(C) Japanese Society of Nephrology 2014

$\begin{array}{ll}\text { Abbreviations } \\ \text { ABI } & \begin{array}{l}\text { ankle-brachial pressure index } \\ \text { ambulatory blood pressure }\end{array} \\ \text { ABP } & \begin{array}{l}\text { ambulatory blood pressure monitoring } \\ \text { ABPM }\end{array} \\ \text { ACEI } & \begin{array}{l}\text { angiotensin converting enzyme inhibitor } \\ \text { ACR }\end{array} \\ \text { ADMA } & \text { asymmetric dimethylarginine } \\ \text { ADPKD } & \text { autosomal dominant polycystic kidney } \\ & \text { disease } \\ \text { AER } & \text { albumin excretion rate } \\ \text { AHA } & \text { American Heart Association } \\ \text { aHR } & \text { adjusted hazard ratio } \\ \text { Alb } & \text { albumin } \\ \text { ANCA } & \text { anti-neutrophil cytoplasmic antibody } \\ \text { APD } & \text { automated peritoneal dialysis } \\ \text { ARB } & \text { angiotensin II type 1 receptor blocker } \\ \text { ASN } & \text { American Society of Nephrology } \\ \text { ASO } & \text { arterio sclerosis obliterans } \\ \text { BMI } & \text { body mass index } \\ \text { BUN } & \text { blood urea nitrogen } \\ \text { CAPD } & \text { continuous ambulatory peritoneal dialysis } \\ \text { CBP } & \text { clinic blood pressure } \\ \text { CCB } & \text { calcium channel blocker } \\ \text { CCr } & \text { creatinine clearance } \\ \text { CHD } & \text { coronary heart disease } \\ \text { CHF } & \text { congestive heart failure } \\ \text { CHF } & \text { continuous hemofiltration } \\ \text { CI } & \text { confidence interval } \\ & \end{array}$

\begin{tabular}{|c|c|}
\hline CKD & chronic kidney disease \\
\hline CKD-MBD & $\begin{array}{l}\text { chronic kidney disease-mineral and bone } \\
\text { disorder }\end{array}$ \\
\hline $\mathrm{Cr}$ & creatinine \\
\hline CRP & $\mathrm{C}$ reactive protein \\
\hline CVD & cardiovascular disease \\
\hline CyA & cyclosporin $\mathrm{A}$, ciclosporin $\mathrm{A}$ \\
\hline DM & diabetes mellitus \\
\hline DOQI & Dialysis Outcome Quality Initiative \\
\hline DRI & direct renin inhibitor \\
\hline DWFG & death with functioning graft \\
\hline EBM & evidence-based medicine \\
\hline eGFR & estimated glomerular filtration rate \\
\hline EPS & encapsulating peritoneal sclerosis \\
\hline ESA & erythropoiesis sitmulating agent \\
\hline ESKD & end-stage kidney disease \\
\hline ESRD & end-stage renal disease \\
\hline FDA & Food and Drug Administration \\
\hline FF & filtration fraction \\
\hline FPG & fasting plasma glucose \\
\hline FSGS & focal segmental glomerulosclerosis \\
\hline GBM & glomerular basement membrane \\
\hline GFR & glomerular filtration rate \\
\hline $\mathrm{GH}$ & growth hormone \\
\hline HCFA & Health Care Financing Administration \\
\hline HD & hemodialysis \\
\hline HR & hazard ratio \\
\hline IBW & ideal body weight \\
\hline IDDM & insulin dependent diabetes mellitus \\
\hline IDF & International Diabetes Federation \\
\hline IDMS & isotope-dilution mass spectrometory \\
\hline IMT & intima-media thickness \\
\hline ISKDC & $\begin{array}{l}\text { International Study of Kidney Disease } \\
\text { in Children }\end{array}$ \\
\hline IVP & intravenous pyelography \\
\hline
\end{tabular}

Japanese Society of Nephrology $(\square)$

Nichinai-Kaikan 6F, 3-28-8 Hongo, Bunkyo-ku,

Tokyo 113-0033, Japan

e-mail: office@jsn.or.jp 


\begin{tabular}{|c|c|c|}
\hline K/DOQI & Kidney Diseases Outcome Quality & waist circumference \\
\hline & Initiative & World Health Organization \\
\hline KDIGO & $\begin{array}{l}\text { Kidney Disease: Improving Global } \\
\text { Outcome }\end{array}$ & waist-to-hip ratio \\
\hline $\mathrm{LVH}$ & left ventricular hypertrophy & Chanter 1: Diagnosis and significance of CKD \\
\hline LVMI & left ventricular mass index & \\
\hline MCDK & multicystic dysplastic kidney & CO1 Is CKD a risk factor for ESKD? \\
\hline MetS & metabolic syndrome & CQ1 IS CND a IISK TACtOI IOI ESND? \\
\hline MMF & mycophenolate mofetil & \\
\hline MMR & vaccine for measles, mumps and rubella & \\
\hline mPSL & methyl prednisolone & A decreased GFR $\left(50 \mathrm{ml} / \mathrm{min} / 1.73 \mathrm{~m}^{2}\right.$ or lower for \\
\hline MRA & magnetic resonance angiography & ged 40 to 69 years and $40 \mathrm{ml} / \mathrm{min} / 1.73 \mathrm{~m}^{2}$ \\
\hline MRSA & methicillin-resistant Staphylococcus aureus & or lower for patients aged 70 years and over), pro- \\
\hline NAFLD & non-alcoholic fatty liver disease & \\
\hline NIDDN & non-insulin-dependent diabetes mellitus & \\
\hline NKF & National Kidney Foundation & \\
\hline NNT & number needed to treat & CKD was defined for the first time in one of the clinical \\
\hline NSAIDs & non-steroidal anti-inflammatory drugs & guidelines of the K/DOQI published in 2002 by NKF. CKD \\
\hline NSF & nephrogenic systemic fibrosis & stages $3-5$ have been known as risk factors for ESKD. In \\
\hline NSTEMI & $\begin{array}{l}\text { non-ST-segment elevation myocardial } \\
\text { infarction }\end{array}$ & $\begin{array}{l}\text { the Japanese population, eGFR } \leq 50 \mathrm{ml} / \mathrm{min} / \mathrm{m}^{2} \text { in patients } \\
\text { aged } 40-69 \text { years and } 40 \mathrm{ml} / \mathrm{min} / 1.73 \mathrm{~m}^{2} \text { in patients aged }\end{array}$ \\
\hline oGTT & oral glucose tolerance test & 70 years and over are risk factors for ESKD. Proteinuria \\
\hline OR & odds ratio & and albuminuria are also proportionally related to the risk \\
\hline PKD & polycystic kidney disease & for ESKD. A meta-analysis of 11 observational studies of \\
\hline PD & peritoneal dialysis & non-diabetic nephropathy indicated that proteinuria before \\
\hline PET & preemptive transplantation & treatment was a strong prognostic factor for the doubling of \\
\hline PHA & proper hepatic artery & serum creatinine and ESKD. This finding could be \\
\hline PP & plasmapheresis & extrapolated to a normal population and pretreated CKD \\
\hline PSL & prednisolone & patients and those on current treatment. Decreased pro- \\
\hline PWV & pulse wave velocity & teinuria and albuminuria by RAS inhibitors are implicated \\
\hline QALY & quality-adjusted life-years & in the suppression of progression of CKD. \\
\hline QOL & quality of life & \\
\hline RA & renin-angiotensin & Bibliography \\
\hline RAS & renin-angiotensin system & 1. Drey N, et al. Am J Kidney Dis. 2003;42:677-84. (Level 4) \\
\hline RBF & renal blood flow & 2. Keith DS, et al. Arch Intern Med. 2004; 164:659-63. (Level 4) \\
\hline $\mathrm{RCT}$ & randomized controlled trial & 3. Patel UD, et al. Am J Kidney Dis. 2005;46:406-14. (Level 4) \\
\hline rHuEPO & recombinant human erythropoietin & 4. Evans M, et al. Am J Kidney Dis. 2005;46:863-70. (Level 4) \\
\hline $\mathrm{rHuGH}$ & recombinant human growth hormone & 5. Eriksen BO, et al. Kidney Int. 2006;69:375-82. (Level 4) \\
\hline ROC & receiver operating curve & 6. Kovesdy CP, et al. Adv Chronic Kidney Dis. 2006;13:183-8. \\
\hline ROD & renal osteodystrophy & (Level 4) \\
\hline RPGN & rapidly progressive glomerulonephritis & $\begin{array}{l}\text { 7. Norris KC, et al. J Am Soc Nephrol. 2006;17:2928-36. (Level } \\
\text { 4) }\end{array}$ \\
\hline $\mathrm{RR}$ & relative risk & 8. Serrano A, et al. Adv Chronic Kidney Dis. 2007;14:105-12. \\
\hline RRT & renal replacement therapy & (Level 4) \\
\hline SBP & systolic blood pressure & 9. Imai E, et al. Hypertens Res. 2008;31:433-41. (Level 4) \\
\hline SLE & systemic lupus erythematosus & 10. Wu MJ, et al. J Chin Med Assoc. 2010;73:515-22. (Level 4) \\
\hline TDM & therapeutic drug monitoring & 11. Levey AS, et al. Kidney Int. 2011;80:17-28. (Level 4) \\
\hline TIA & transient cerebral ischemic attack & 12. Iseki K, et al. Kidney Int. 2003;63:1468-74. (Level 4) \\
\hline TIBC & total iron binding capacity & 13. Zhang Z, et al. J Am Soc Nephrol. 2005;16:1775-80. (Level 4) \\
\hline TSAT & transferrin saturation & 14. Peterson JC, et al. Ann Intern Med. 1995;123:754-62. (Level 4) \\
\hline UACR & urinary albumin/creatinine ratio & 15. Lea J, et al. Arch Intern Med. 2005;165:947-53. (Level 4) \\
\hline UAE & urinary albumin excretion & 16. Halbesma N, et al. J Am Soc Nephrol. 2006;17:2582-90. (Level \\
\hline VUR & vesicoureteral reflux & $\begin{array}{l}\text { 4) } \\
\text { 17. Jafar TH, et al. Kidney Int. 2001;60:1131-40. (Level 1) }\end{array}$ \\
\hline
\end{tabular}


CQ2 Is CKD a risk factor for CVD?

Decreased kidney function is a risk factor for CVD. Increased proteinuria and albuminuria are also associated with a higher risk of CVD.

ESKD patients are known to be at increased risk of CVD. Earlier intervention for CKD has been recognized as more important for the prevention of CVD. A scientific statement entitled, "Kidney Disease as a Risk Factor for the Development of Cardiovascular Disease" prompted heightened attention to CVD as a complication resulting in evidence that the early stage of CKD as well as ESKD are both risk factors for CVD. GFR decline is correlated to the risk of CVD, coronary disease, myocardial infarction, heart failure, atrial fibrillation, stroke, admission, mortality from CVD and total death. Proteinuria and albuminuria also increase the risk. Several large-scale observational studies using a normal population in Japan have also indicated CKD to be a risk factor for CVD.

\section{Bibliography}

1. Kannel WB, et al. Am Heart J. 1984;108:1347-52. (Level 4)

2. Damsgaard EM, et al. BMJ. 1990;300:297-300. (Level 4)

3. Sarnak MJ, et al. Circulation. 2003;108:2154-269. (Level 1)

4. Keith DS, et al. Arch Intern Med. 2004;164:659-63. (Level 4)

5. Go AS, et al. N Engl J Med. 2004;351:1296-305. (Level 4)

6. Ninomiya T, et al. Kidney Int. 2005;68:228-36. (Level 4)

7. Anavekar NS, et al. N Engl J Med. 2004;351:1285-95. (Level 4)

8. Fox CS, et al. Circulation. 2010;121:357-65. (Level 4)

9. Kottgen A, et al. J Am Soc Nephrol. 2007;18:1307-15. (Level 4)

10. Brugts JJ, et al. Arch Intern Med. 2005;165:2659-65. (Level 4)

11. Nitsch D, et al. Am J Kidney Dis. 2011;57:664-72. (Level 4)

12. Brown JH, et al. Nephrol Dial Transplant. 1994;9:1136-42. (Level 4)

13. Horio T, et al. J Hypertens. 2010;28:1738-44. (Level 4)

14. Nakayama M, et al. Nephrol Dial Transplant. 2007;22:1910-5. (Level 4)

15. Weiner DE, et al. J Am Soc Nephrol. 2007;18:960-6. (Level 4)

16. Ovbiagele B. J Neurol Sci. 2011;301:46-50. (Level 4)

17. Drey N, et al. Am J Kidney Dis. 2003;42:677-84. (Level 4)

18. Irie F, et al. Kidney Int. 2006;69:1264-71. (Level 4)

19. Nakamura K, et al. Circ J. 2006;70:954-9. (Level 4)

20. Ninomiya T, et al. Circulation. 2008;118:2694-701. (Level 4)

21. Kokubo Y, et al. Stroke. 2009;40:2674-9. (Level 4)

CQ3 Is the prognosis determined by the definition and classification of CKD (KDIGO 2011)?

The classification of CKD (KDIGO 2011) correlates with the progression of CKD, ESKD, mortality due to CVD and total death, and thus reflects the prognosis.
The definition and classification of CKD (NKF-KDOQI) were first proposed in 2002 and have not been revised since 2009 , hence their current validity requires discussion as 8.4 and $12.9 \%$ of the population in the United States and Japan, respectively, are diagnosed as CKD on the basis of that definition.

Several meta-analyses have shown significant correlation between CKD staging and prognosis. The CKD classification (KDIGO 2011) divided stage 3 into G3a and G3b at the eGFR level of $45 \mathrm{ml} / \mathrm{min} / 1.73 \mathrm{~m}^{2}$, since risks for the progression of CKD sharply increase at this point. In Japan, since the same tendency was observed, the eGFR level of $50 \mathrm{ml} / \mathrm{min}$ is proposed as the criterion for referral to a specialist. (criteria by age; an eGFR level of $60 \mathrm{ml} / \mathrm{min} /$ $1.73 \mathrm{~m}^{2}$ for patients aged less than 40 years, an eGFR level of $50 \mathrm{ml} / \mathrm{min} / 1.73 \mathrm{~m}^{2}$ for patients aged $40-69$ years, and an eGFR level of $40 \mathrm{ml} / \mathrm{min} / 1.73 \mathrm{~m}^{2}$ for patients aged 70 years or more).

The albuminuria category was introduced into the classification of CKD (KDIGO 2011). However, as albuminuria is covered by Japanese health insurance only for early diabetes nephropathy, we decided to use albuminuria for diabetes and proteinuria for the others (Table 1).

\section{Bibliography}

1. Levey AS, et al. Kidney Int. 2011;80:17-28. (Level 4)

2. Chronic Kidney Disease Prognosis Consortium. Lancet. 2010;375:2073-81. (Level 4)

3. Imai E, et al. Hypertens Res. 2008;31:433-41. (Level 4)

4. Steinman MA, et al. J Am Soc Nephrol. 2006;17:846-53. (Level 4)

CQ4 Is the guideline based on the definition and classification of CKD (KDIGO 2011) recommended?

Dividing CKD stage 3 into G3a and G3b is recommended as it facilitates early therapeutic intervention in stage G3b patients whose kidney dysfunction is easily aggravated. CKD classification with the albuminuria category is recommended because it clarifies those cases who are at high risk for CVD, and in whom RAS inhibitors are effective.

Dividing stage 3 and use of the albuminuria category are characteristics of the classification of CKD (KDIGO 2011). The advantage of this classification in the treatment strategy is discussed. Clinical diagnosis determines the diseasespecific treatment, whereas general treatment is based on the classification of CKD. The reason for dividing stage 3 into G3a and G3b is that the category with an eGFR level 
Table 1 Classification of severity of CKD (2012)

\begin{tabular}{|c|c|c|c|c|c|c|}
\hline \multicolumn{2}{|c|}{ Underlying disease } & \multicolumn{2}{|c|}{$\begin{array}{l}\text { Albuminuria categories, } \\
\text { description, and range }\end{array}$} & A1 & A 2 & A3 \\
\hline \multirow{2}{*}{\multicolumn{2}{|c|}{ Diabetes }} & \multirow{2}{*}{\multicolumn{2}{|c|}{$\begin{array}{l}\text { Urine albumin (mg/day) } \\
\text { Urine albumin/Cr ratio } \\
\qquad(\mathrm{mg} / \mathrm{gCr})\end{array}$}} & Normal & Microalbuminuria & Macroalbuminuria \\
\hline & & & & $<30$ & $30 \sim 299$ & $\geq 300$ \\
\hline \multirow{2}{*}{\multicolumn{2}{|c|}{$\begin{array}{l}\text { Nephritis } \\
\text { Hypertension } \\
\text { Polycystic kidney disease } \\
\text { Renal transplantation } \\
\text { Unknown } \\
\text { Others }\end{array}$}} & \multicolumn{2}{|c|}{ Urine protein (g/day) } & Normal & Mild proteinuria & Severe proteinuria \\
\hline & & \multicolumn{2}{|c|}{$\begin{array}{l}\text { Urine protein/Cr ratio } \\
\qquad(\mathrm{g} / \mathrm{gCr})\end{array}$} & $<0.15$ & $0.15 \sim 0.49$ & $\geq 0.50$ \\
\hline \multirow{6}{*}{$\begin{array}{c}\text { GFR categories } \\
\left(\mathrm{mL} / \mathrm{min} / 1.73 \mathrm{~m}^{2}\right), \\
\text { description, and } \\
\text { range }\end{array}$} & G1 & Normal or high & $\geq 90$ & & & \\
\hline & G2 & $\begin{array}{c}\text { Normal or mildly } \\
\text { decreased }\end{array}$ & $60 \sim 89$ & & & \\
\hline & G3a & $\begin{array}{l}\text { Mildly to } \\
\text { moderately } \\
\text { decreased }\end{array}$ & $45 \sim 59$ & & & \\
\hline & G3b & $\begin{array}{c}\text { Moderately to } \\
\text { severely } \\
\text { decreased }\end{array}$ & $30 \sim 44$ & & & \\
\hline & G4 & $\begin{array}{l}\text { Severely } \\
\text { decreased }\end{array}$ & $15 \sim 29$ & & & \\
\hline & G5 & $\begin{array}{l}\text { Kidney failure } \\
\text { (ESKD) }\end{array}$ & $<15$ & & & \\
\hline
\end{tabular}

Risks of ESKD requiring dialysis, or transplantation, and risks for cardiovascular diseases such as stroke, myocardial infarction, and heart failure are coded with colors ranging from green (lowest), yellow, orange and red (highest)

Adapted from KDIGO 2012 Clinical Practice Guideline for the Evaluation and Management of Chronic Kidney Disease. Kidney Inter Suppl. 2013;3:19-62 [1], with permission from Nature Publishing Group, modified for Japanese patients

$C K D$ chronic kidney disease, $C r$ creatinine, $E S K D$ end-stage kidney disease, GFR glomerular filtration

of less than $45 \mathrm{ml} / \mathrm{min} / 1.73 \mathrm{~m}^{2}$ has sharply increased risks of progression of CKD and ESKD. In the stage G4 category, hypertension, anemia, secondary parathyroidism, and electrolyte abnormality such as hyperphosphatemia, acidosis and hypoalbuminemia are commonly observed. The sub-division of stage G3 is efficient for avoiding such complications, preventing the progression of CKD stage, and facilitating consultation with a specialist at the appropriate time point. The albuminuria category is clinically useful because RAS inhibitors are more effective in CKD patients with albuminuria and proteinuria.

\section{Bibliography}

1. Levey AS, et al. Kidney Int. 2011;80:17-28. (Level 4)

2. Moranne O, et al. J Am Soc Nephrol. 2009;20:164-71. (Level 4)
3. Nakamura S, et al. Circ J. 2007;71:511-6. (Level 4)

4. Black C, et al. Health Technol Assess. 2010;14:1-184. (Level 4)

5. Casas JP, et al. Lancet. 2005;366:2026-33. (Level 1)

6. Jafar TH, et al. Ann Intern Med. 2003;139:244-52. (Level 4)

7. Ibsen H, et al. Hypertension. 2005;45:198-202. (Level 4)

CQ5 Which urine test, albumin or total protein, is recommended to properly manage $\mathrm{CKD}$ ?

We recommend albumin measurement for the early detection and risk evaluation of diabetic nephropathy. Total protein measurement may have an advantage in clinical practice for advanced diabetic nephropathy and non-diabetic CKD. 
Proteinuria in CKD is one of the important prognostic factors. Albuminuria in the traditional "normal range" has been revealed as an apparent risk factor of CVD. Meanwhile, total protein is recommended for non-diabetic CKD in several countries. In Japan, albuminuria is not covered by the health insurance system.

Whereas albumin in urine is derived from the glomerulus, total protein consists of a variety of proteins derived from the glomerulus and renal tubules. The amount of high-molecular-weight protein correlates with the prognosis of kidney function. Recently, the sensitivity of detection of total protein at concentrations less than $0.5 \mathrm{~g} / \mathrm{gCr}$ has become more accurate. Consequently, albumin measurement is recommended for the early detection and risk evaluation of the early stage of diabetic nephropathy. Total protein measurement is recommended for advanced diabetic nephropathy and non-diabetic CKD. Although in Japan, the HPLC/ultraviolet detection method using $99 \%$ pure human plasma albumin as the primary standard substance for total protein measurement is recommended, the pigment colorimetric method is in wide practical use and is accurate using human plasma albumin as the standard substance.

\section{Bibliography}

1. Gerstein HC, et al. JAMA. 2001;286:421-6. (Level 4)

2. Wachtell K, et al. Ann Intern Med. 2003;139:901-6. (Level 4)

3. Arnlov J, et al. Circulation. 2005;112:969-75. (Level 4)

4. Bazzi C, et al. Kidney Int. 2000;58:1732-41. (Level 4)

5. Tencer J, et al. Clin Chim Acta. 2000;297:73-83. (Level 4)

6. Methven S, et al. QJM. 2011;104(8):663-70. (Level 4)

7. Methven S, et al. Nephrol Dial Transplant. 2010;25:2991-6. (Level 4)

CQ6 What is a useful urinary clinical surrogate marker for following the clinical course of CKD?

Urinary protein excretion and urinary albumin excretion are recommended biomarkers for evaluating the prognosis of CKD. Other promising biomarkers would be urinary excretions of $\alpha 1$ microglobulin, $\beta 2$ - microglobulin and L-FABP.

At present, urinary excretion of protein or albumin is considered to be a useful biomarker for the assessment of CKD (refer to CQ5), although biomarkers other than proteinuria or albuminuria for CKD have not yet been fully evaluated for their usefulness.

The results of two clinical studies on the prognosis of idiopathic membranous nephropathy showed that urinary excretions of both $\alpha 1$-microglobulin and $\beta 2$-microglobulin were significantly associated with the prognosis of renal function.

Although L-FABP was reported to be a novel biomarker for AKI, urinary excretion of L-FABP was associated with albuminuria levels and was also associated with the prognosis of renal function in 140 diabetic nephropathy patients who were followed for 4 years. Measurement of urinary excretions of L-FABP was admitted officially for clinical practice and the cost has been partially covered by the health insurance system since August, 2011.

\section{Bibliography}

1. Hofstra JM, et al. Nephrol Dial Transplant. 2008;23:2546-51. (Level 4)

2. van den Brand JA, et al. Clin J Am Soc Nephrol. 2011;6:2846-53. (Level 4)

3. Kamijo-Ikemori A, et al. Diabetes Care. 2011;34:691-6. (Level 4)

4. Hofstra JM, et al. Nephrol Dial Transplant. 2008;23:3160-5. (Level 4)

5. Bolignano D, et al. Clin J Am Soc Nephrol. 2009;4:337-44. (Level 4)

6. Idasiak-Piechocka I, et al. Nephrol Dial Transplant. 2010;25:3948-56. (Level 4)

7. Idasiak-Piechocka I, et al. Nephron Clin Pract. 2010;116:c47c52. (Level 4)

8. O'Seaghdha CM, et al. Am J Kidney Dis. 2011;57:841-9. (Level 4)

CQ7 Does the severity of hematuria predict renal prognosis?

Microscopic hematuria is an independent risk factor for progression to ESKD, although it presents a lower risk than proteinuria. Therefore, we recommend follow-up of hematuria in a regular medical health checkup.

The risk for progression to ESKD increases when hematuria coexists with proteinuria.

A recent Israeli cohort study of 1,203,626 military soldiers aged 16-25 years revealed the possibility of isolated hematuria progressing to ESKD to be $0.7 \%$ and the hazard ratio to be 19.5 compared to normal urinary findings. A 10-year observational study based on the findings of regional health checkups of 107,192 subjects revealed that $0.2 \%$ of the subjects progressed to ESKD and that hematuria was identified as an independent risk factor for the progression. Analysis using the same cohort showed that the probability of subjects with both proteinuria at the level of $1+$ and hematuria at the level of $1+$ progressing to ESKD within 10 years increased to $3 \%$, while the probability in patients with isolated proteinuria was $1.5 \%$. A cohort study of 50,501 company employees showed that 
hematuria spontaneously remitted in half of the subjects with isolated hematuria and that $10 \%$ of isolated hematuria cases became complicated with proteinuria. In conclusion, even in subjects with isolated hematuria, regular checkups should be mandatory to monitor potential complication with proteinuria in the future.

\section{Bibliography}

1. Chow KM, et al. QJM. 2004;97:739-45. (Level 4)

2. Kim BS, et al. Korean J Intern Med. 2009;24:356-61. (Level 4)

3. Vivante A, et al. JAMA. 2011;306:729-36. (Level 4)

4. Iseki K, et al. Kidney Int. 1996;49:800-5. (Level 4)

5. Iseki K. J Am Soc Nephrol. 2003;14:S127-30. (Level 4)

6. Yamagata K, et al. Clin Nephrol. 1996;45:281-8. (Level 4)

7. Yamagata K, et al. Nephron. 2002;91:34-42. (Level 4)

8. Goto M, et al. Nephrol Dial Transplant. 2009;24:3068-74. (Level 4)

9. Manno C, et al. Am J Kidney Dis. 2007;49(6):763-75. (Level 4)

10. Rauta V, et al. Clin Nephrol. 2002;58:85-94. (Level 4)

11. Daniel L, et al. Am J Kidney Dis. 2000;35:13-20. (Level 4)

12. Johnson AM, et al. J Am Soc Nephrol. 1997;8:1560-7. (Level 4)

CQ8 Is renal biopsy recommended for determining the diagnosis and therapeutic strategy for CKD?

After considering the results of a urine test (Table 2) for reference, we recommend a renal biopsy in order to determine the diagnosis and therapeutic strategy for CKD.

Evaluating renal pathology by a renal biopsy is of great help in determining the therapeutic strategy and estimating the long-term prognosis. In this regard, a renal biopsy is recommended in CKD clinical practice. However, since a renal biopsy is invasive, its use should be considered carefully. It has been shown that CKD patients that have undergone a renal biopsy have a better mortality after the introduction of hemodialysis than those who have not. Moreover, the percentage of cases in whom the results of renal biopsy had some impact on the clinical course was $86 \%$ (24 cases out of 28 ) in patients with nephrotic syndrome, $71 \%$ (22 out of 31 ) in AKI, $45 \%$ (9 out of 28 ) in asymptomatic hematuria or proteinuria, $12 \%$ (3 out of 25 ) in isolated proteinuria, $3 \%$ ( 1 out of 36 ) in isolated hematuria, and $42 \%$ in all the patients examined. These data point to the importance of the information obtained from a renal biopsy for the care of CKD patients, although these data might not necessarily show that a renal biopsy leads to a favorable prognosis.
A Japanese nation-wide surveillance study found that $50 \%$ of nephrologists thought that a biopsy should be performed in patients with isolated proteinuria and whose daily protein excretion was over $1 \mathrm{~g}$, and that $75 \%$ of nephrologists thought that it should be performed on patients complicated with hematuria and whose daily protein excretion was over $0.5 \mathrm{~g}$. Taken together, it is reasonable to conclude that that a renal biopsy should be performed on patients with sustained proteinuria at a level above $0.5 \mathrm{~g} /$ day (Table 2).

\section{Bibliography}

1. Iseki K, et al. Kidney Int. 2004;66:914-9. (Level 4)

2. Ferro G, et al. Clin Nephrol. 2006;65:243-7. (Level 4)

3. Iseki K, et al. Kidney Int. 2003;63:1468-74. (Level 4)

4. Fuiano G, et al. Am J Kidney Dis. 2000;35:448-57. (Level 4)

5. Biesenbach G, et al. QJM. 2011;104:771-4. (Level 4)

6. Suzuki D, et al. Intern Med. 2001;40:1077-84. (Level 4)

7. Sugiyama H, et al. Clin Exp Nephrol. 2011;15:493-503. (Level 4)

8. Le W, et al. Nephrol Dial Transplant. 2012;27:1479-85. (Level 4)

CQ9 Is medical imaging recommended for the diagnosis of CKD?

In the diagnosis of $\mathrm{CKD}$, we recommend ultrasonography for the diagnosis of renal disease with a morphological abnormality (e.g. urinary stone, obstructive nephropathy, urinary cystic disease).

In the diagnosis of renal artery stenosis, we recommend Doppler ultrasonography, MR angiography, or $\mathrm{CT}$ angiography after considering the patient's renal function.

Several modalities, including ultrasonography, abdominal CT, and abdominal MRI have been utilized for the diagnostic imaging of kidney disease. Among these, because of its convenience and lack of exposure to

Table 2 Use of renal biopsy in CKD patients

Isolated proteinuria
Should be considered when daily urinary excretion is more than
$0.5 \mathrm{~g} /$ day or $0.5 \mathrm{~g} / \mathrm{gCr}$
Proteinuria and hematuria
Should be considered even when daily urinary excretion is less
than $0.5 \mathrm{~g} /$ day or $0.5 \mathrm{~g} / \mathrm{gCr}$
Nephrotic syndrome
Should always be considered
Isolated hematuria
Should be considered when urine contains dysmorphic
erythrocytes or abnormal urinary casts


radiation, ultrasonography should be performed on all types of renal diseases, especially those with morphological abnormalities (e.g. urinary stone, obstructive nephropathy, urinary cystic disease).

Diagnostic imaging can be a useful tool for the diagnosis of renal artery stenosis or ischemic nephropathy caused by chronic reduction of renal perfusion. Although Doppler ultrasonography is inferior to CT angiography, Gadolinium-enhanced MR angiography and three-dimensional MRI in ROC evaluation, it is still a useful tool on account of its convenience and economical cost.

\section{Bibliography}

1. Vasbinder GB, et al. Ann Intern Med. 2001;135:401-11. (Level 4)

2. Williams GJ, et al. AJR Am J Roentgenol. 2007;188:798-811. (Level 4)

3. Nakamura S, et al. Hypertens Res. 2007;30:839-44. (Level 4)

4. Burdick L, et al. J Hypertens. 1996;14:1229-35. (Level 4)

5. Ripollés T, et al. Eur J Radiol. 2001;40:54-63. (Level 4)

6. Zeller T, et al. Circulation. 2003;108:2244-9. (Level 4)

7. Inoue T, et al. J Am Soc Nephrol. 2011;22:1429-34. (Level 4)

8. Perrone RD, et al. Am J Kidney Dis. 1990;16:224-35. (Level 4)

9. Ma YC, et al. Nephrol Dial Transplant. 2007;22:417-23. (Level 4)

CQ10 Is a regular health checkup useful for the early diagnosis of CKD?

For the diagnosis of CKD and evaluation of CKD staging, measurements of both urinary protein or albumin excretion and serum creatinine are indispensable. Since most renal diseases start without any symptoms, urinalysis and serum creatinine measurement in a regular health checkup are useful for the early diagnosis of CKD.

For patients at high risk of CKD including those with hypertension, diabetes, obesity, or metabolic syndrome and those who had previous CVD events, measurement of proteinuria and serum creatinine concentration should be performed at least once a year.

In the diagnosis of CKD and the classification of CKD staging, measurement of urinary protein or albumin excretion and serum creatinine are mandatory. Numerous papers have indicated the beneficial effects of the Japanese health system in which urinary protein excretion and serum creatinine measurement lead to the early diagnosis of CKD. A recent report analyzed the cost-effectiveness of measuring serum creatinine in an annual health checkup for preventing the initiation of maintenance dialysis. It revealed that the total cost of measuring proteinuria and serum creatinine for preventing the initiation of maintenance dialysis in ESKD patients was 10 million yen per subject, which could be covered by the budget of developed countries.

\section{Bibliography}

1. Chronic Kidney Disease Prognosis Consortium. Lancet. 2010;375:2073-81. (Level 4)

2. Irie F, et al. Kidney Int. 2006;69:1264-71. (Level 4)

3. Iseki K, et al. Kidney Int. 1996;49:800-5. (Level 4)

4. Iseki K, et al. Clin Exp Nephrol. 2012;16:244-9. (Level 4)

5. Kondo M, et al. Clin Exp Nephrol. 2012;16:279-91. (Level 4)

\section{Chapter 2: CKD and Life-style}

CQ1 Does alcohol consumption have an influence on the onset or progression of CKD?

Light to moderate alcohol consumption (ethanol 10-20 g/day) does not decrease the glomerular filtration rate and may decrease proteinuria.

Heavy alcohol consumption (ethanol over 20-30 g/ day) may have the potential to cause proteinuria to develop.

Heavy alcohol consumption is one of the major causes of liver disease, cancer, suicide, and traffic accidents. Recently, light to moderate alcohol consumption has been shown to reduce coronary heart disease and all-cause mortality. We aimed to clarify the relationship between alcohol consumption and CKD.

1. Incidence of urinary protein

In Japan, alcohol consumption of less than 20 g/day decreased the hazard ratio [0.86 (95\%CI 0.78-0.95)] of developing proteinuria, but this effect was diminished by alcohol consumption of more than $20 \mathrm{~g} /$ day. However, it was found that moderate to heavy alcohol consumption may be an important modifiable risk factor for albuminuria in the general population in Australia.

2. Estimated glomerular filtration rate (estimated GFR) Funakoshi et al. reported that significant differences in the frequency of drinking alcohol were found to be inversely related to the estimated GFR and the prevalence of CKD in Japanese men. However, the relationship was not observed in the elderly and Shankar et al. reported that smoking and consumption of 4 or more glasses of alcohol per day were associated with CKD.

Attention should be paid to the quantity of the participants' alcohol consumption because interviews or questionnaires may lead to incorrect estimates of alcohol consumption (Tables 3, 4). 
Table 3 Quantity of alcohol in the standard size for each alcohol

\begin{tabular}{|c|c|c|c|c|}
\hline Alcohol & Size & $\mathrm{ml}$ & $\%$ & $\begin{array}{l}\text { Ethanol } \\
(\mathrm{g})\end{array}$ \\
\hline Beer & 1 medium bottle & 500 & 5 & 20 \\
\hline Sake (Japanese rice wine) & $\begin{array}{l}1 \text { go (Japanese } \\
\text { unit) }\end{array}$ & 180 & 15 & 22 \\
\hline Whisky or Brandy & double & 60 & 43 & 20 \\
\hline $\begin{array}{l}\text { Shochu (Japanese liquor } \\
35^{\circ} \text { ) }\end{array}$ & $\begin{array}{l}1 \text { go (Japanese } \\
\text { unit) }\end{array}$ & 180 & 35 & 50 \\
\hline Wine & 1 glass & 129 & 12 & 12 \\
\hline
\end{tabular}

CKD clinical guidelines 2009

Table 4 Quantity of alcohol in a standard drink of each country

O'Shea RS, et al. Alcoholic liver disease. Hepatology. 2010;51(1):307-28

\begin{tabular}{lll}
\hline Country & $\begin{array}{l}\text { Ethanol } \\
(\mathrm{g})\end{array}$ & Range $(\mathrm{g})$ \\
\hline USA & 12 & $9.3-13.2$ \\
Canada & 13.6 & 13.6 \\
UK & 9.5 & $8-10$ \\
Europe & 9.8 & $8.7-10.0$ \\
AUS & 9.2 & $6.0-11.0$ \\
$\quad$ and & & \\
$\quad$ NZ & & \\
Japan & 23.5 & $21.2-28.0$ \\
\hline
\end{tabular}

\section{Bibliography}

1. White SL, et al. Nephrol Dial Transplant. 2009;24:2464-72. (Level 4)

2. Yamagata K, et al. Kidney Int. 2007;71:159-66. (Level 4)

3. Funakoshi Y, et al. Environ Health Prev Med. 2012;17:199-204. (Level 4)

4. Menon V, et al. Nephrol Dial Transplant. 2010;25:3301-7. (Level 4)

5. Shankar A, et al. Am J Epidemiol. 2006;164:263-71. (Level 4)

6. Knight EL, et al. Nephrol Dial Transplant. 2003;18:1549-54. (Level 4)

7. Reynolds K, et al. Kidney Int. 2008;73:870-6. (Level 4)

8. Schaeffner ES, et al. Arch Intern Med. 2005;165:1048-53. (Level 4)

CQ2 Does exercise affect the onset or progress of CKD?

The effect of exercise on the onset or progress of CKD is unknown.

Inactivity and lower health-related quality of life (HRQOL) are regarded as risk factors for mortality and hospitalization in patients with dialysis. However, little has been reported about the effect of exercise on the onset or progress of CKD.
Heiwe et al. reported in a systematic review (45 studies with 1863 adult participants with CKD) that there was evidence for significant beneficial effects of regular exercise on physical fitness, walking capacity, cardiovascular dimensions (e.g. blood pressure and heart rate), HRQOL and some nutritional parameters. However, the result of the relationship between exercise and urinary protein or GFR was controversial.

For obese patients with CKD, exercise improved body weight, blood pressure and urinary protein.

The risk of cardiac events (arrhythmia, ischemic heart disease, and sudden death) during exercise is well known in patients with CKD. Therefore, when patients are prescribed exercise, it is essential to assess every patient's activity, exercise tolerance, and risk of cardiovascular disease.

\section{Bibliography}

1. Heiwe $\mathrm{S}$, et al. Cochrane Database Syst Rev. 2011;10:CD003236. (Level 1)

2. Leehey DJ, et al. Cardiovasc Diabetol. 2009;8:62. (Level 2)

3. Pechter U, et al. Int J Rehabil Res. 2003;26:153-6. (Level 4)

4. Kosmadakis GC, et al. Nephrol Dial Transplant. 2012;27(3):9971004. (Level 3)

5. Eidemak I, et al. Nephron. 1997;75:36-40. (Level 2)

6. Boyce ML, et al. Am J Kidney Dis. 1997;30:180-92. (Level 4)

7. Afshinnia F, et al. Nephrol Dial Transplant. 2010;25:1173-83. (Level 3)

CQ3 Does sleep affect the onset or progression of CKD?

Short sleep duration and or sleep disorder may be associated with the incidence of proteinuria and decrease in the glomerular filtration rate.

Sleep apnea syndrome is closely associated with CKD.

Short sleep duration or sleep disorder may be associated with hypertension, diabetes mellitus, obesity, cardiovascular disease, and sudden death. However, an association between short sleep duration or sleep disorder and CKD is unclear in patients with CKD.

1. Sleep duration

Sleep duration was short in patients with CKD (338 $\pm 96 \mathrm{~min}$ ) compared with the control (non-CKD $366 \pm 67 \mathrm{~min}$ ). Short sleep duration, especially 5 or fewer hours, was a predictor of proteinuria in Japan.

2. Sleep quality

Sleep quality assessed by the Pittsburg Sleep Quality Index (PSQI), was poorer in participants with CKD than in participants with non-CKD. However, the sample size of the participants in these reports was too small to evaluate the sleep quality. 
3. Sleep disorder: sleep apnea syndrome

Caution should be taken when applying the results of overseas studies to the Japanese population, because the mean BMI of the participants has been more than $30 \mathrm{~kg} / \mathrm{m}^{2}$ in most European and American studies on sleep apnea. A high prevalence of CKD was observed among patients with sleep-related breathing disorder in a single Japanese sleep center and there was an inverse relationship between BMI and the prevalence of CKD.

\section{Bibliography}

1. Plantinga L, et al. Association of Sleep-Related Problems with CKD in the United States, 2005-2008. Am J Kidney Dis. 2011;58:554-64. (Level 4)

2. Agarwal R, et al. Clin J Am Soc Nephrol. 2011;6:1258-65. (Level 4)

3. Yamamoto R, et al. Am J Kidney Dis. 2012;59(3):343-55. (Level 4)

4. De Santo RM, et al. Semin Nephrol. 2006;26:64-7. (Level 4)

5. De Santo RM, et al. J Ren Nutr. 2010;20:S59-63. (Level 4)

6. Sabbatini M, et al. Sleep Med. 2008;9:240-6. (Level 4)

7. Iseki K, et al. Hypertens Res. 2008;31:249-55. (Level 4)

8. Sakaguchi Y, et al. Clin J Am Soc Nephrol. 2011;6:995-1000. (Level 4)

CQ4 Does smoking affect the development of CKD?

Smoking is a risk factor for the onset and progression of CKD.

Smoking is well known as a risk factor for cancer and CVD. Moreover, smokers are also reported to be at a high risk for metabolic syndrome, which is related to the development of CKD. A review of the current literature was performed to investigate the relationship between smoking and the development of CKD.

Yamagata et al. reported that smoking is one risk factor for the onset and progression of CKD in the general population of Japan. They conducted a 10-year follow-up study with a total of 123,764 healthy patients aged 40 years and above who received community-based annual examinations. The primary outcome of the analysis was the development of CKD during the follow-up period. They showed that smoking was an independent risk factor for the development of CKD and increased the risk of proteinuria and renal dysfunction in both genders. However, former smoker status was not a risk factor for developing proteinuria or renal dysfunction. This study suggests that quitting smoking would have a favorable effect on preventing the development of CKD.

Another Japanese group (Ishizaki et al.) investigated the association between smoking and albuminuria or glomerular filtration rate (GFR) by analyzing data from 7,078 Japanese men, who had undergone a general health screening between 2005 and 2006. Multivariate logistic regression analysis showed that current smoking habits were positively associated with albuminuria and inversely associated with a low eGFR. The association between smoking and GFR was dependent on the number of cigarettes smoked per day. A history of smoking showed a significant inverse association with a low eGFR, but there was no significant association between former smoking status and albuminuria. These data suggest that smoking may increase albuminuria and decrease eGFR, and that albuminuria may be reversed by quitting smoking.

Stengel et al. examined data from a non-concurrent cohort study of 9,082 US adults, aged 30-74 years, who participated in the second National Health and Nutrition Examination Survey (NHANES II) from 1976 through 1980. The risk of CKD was found to be related to smoking: the relative risk (RR) in smokers of 1-20 cigarettes a day versus never-smokers was $1.2(95 \% \mathrm{CI}$ 0.7-2.3), and in smokers of more than 20 cigarettes a day, the RR rose to 2.3 (95\% CI 1.3-4.2). This study suggests that not only quitting smoking, but also cigarette reduction may reduce the development of kidney disease.

Shankar et al. performed a longitudinal analysis among 3,392 CKD-free persons at baseline, looking at the incidence of CKD $(n=114)$ over a 5 year period. Compared to never-smokers, the odds ratio of developing CKD was 1.12 (95\% CI 0.63, 2.00) among former smokers and 1.97 $(95 \%$ CI 1.15, 3.36) among current smokers.

Haroun et al. performed a community-based, prospective observational study of 20-year duration to examine the association between hypertension and smoking on the future risk of CKD in 23,534 men and women in a local region. The results showed that current smoking was significantly associated with the risk of developing CKD in both men [hazard ratio $2.4(1.5-4.0)$ ] and women [hazard ratio $2.9(1.7-5.0)]$.

Above all, smoking is a risk factor for the development of CKD and proteinuria, and former smokers may improve albuminuria by quitting smoking compared to current smokers. Therefore, it is recommended to quit smoking.

\section{Bibliography}

1. Yamagata K, et al. Kidney Int. 2007;71:159-66. (Level 4)

2. Ishizaka N, et al. Hypertens Res. 2008;31:485-92. (Level 4)

3. Stengel B, et al. Epidemiology. 2003;14:479-87. (Level 4)

4. Shankar A, et al. Am J Epidemiol. 2006;164:263-71. (Level 4)

5. Haroun MK, et al. J Am Soc Nephrol. 2003;14:2934-41. (Level 4) 
CQ5 Does increased water intake affect

the development of CKD?

In patients with CKD stage $\mathrm{G} 1$ or $\mathrm{G} 2$, increased water intake is effective for the maintenance of kidney function.

In patients with CKD stage G3 or higher, increased water intake is a risk factor for worsening kidney function.

The effect of increased water intake on the onset and development of CKD is unclear, but dehydration exacerbates kidney function.

Clark et al. performed a prospective cohort study in Canada from 2002 to 2008. Of 3,371 participants $\geq 18$ years of age, 2,148 provided valid 24 -h urine samples at study entry and had at least two annual eGFR assessments. The participants were then assigned to the following groups: <1 L/day (14.5\%), 1-1.9 L/day (51.5 \%), $2-2.9 \mathrm{~L} /$ day $(26.3 \%)$, and $\geq 3 \mathrm{~L} /$ day $(7.7 \%)$. As water intake increased, the percentage annual eGFR decline turned out to be $1.3,1.0,0.8,0.5 \%$, respectively.

Hebert et al. reported that high fluid intake resulted in an increased urine volume, and low urine osmolality (Uosm) was not associated with slower renal disease progression. In a randomized control trial performed by Spigt et al., one group was advised to increase their daily fluid intake by $1.5 \mathrm{~L}$ of water, and the other group was given placebo medication. Most subjects did not manage to increase their fluid intake by $1.5 \mathrm{~L}$. The average increase in the intervention group was approximately $1 \mathrm{~L}$. Twenty-four-hour water turnover in the intervention group was $359 \mathrm{~mL}$ (95\% CI 171-548) higher than that of the control group at the 6-month follow-up. Blood pressure, sodium level, GFR, and QOL did not change significantly in either group during the intervention period.

Increased water intake is effective for maintaining kidney function in CKD patients at stage G1 and G2, but it could be a risk factor for worsening kidney function in CKD patients at stage G3 and higher. Dehydration can exacerbate kidney function at any CKD stage. It is important to maintain an appropriate water intake based on the CKD stage.

\section{Bibliography}

1. Clark WF, et al. Clin J Am Soc Nephrol. 2011;6:2634-41. (Level 4)

2. Hebert LA, et al. Am J Kidney Dis. 2003;41:962-71. (Level 4)

3. Spigt MG, et al. J Am Geriatr Soc. 2006;54:438-43. (Level 2)
CQ6 Is vaccination recommended for CKD?

We recommend Influenza vaccination for patients with CKD and Pneumococcal vaccination for CKD patients aged over 65 years. (Grade B)

CKD patients have a weakened immune system and are at risk of higher morbidity and mortality rates from infections compared to healthy subjects. It is recommended that CKD patients should be given vaccinations against high risk pathogens. Pneumococcal and Influenza vaccines are inactivated, hence both have a low potential for adverse events related to the administration of the vaccination.

Influenza is a common and widespread infection causing morbidity and mortality in the general population, and regular vaccinations are recommended to prevent the associated comorbidities. Influenza may be significantly exacerbated to pneumonia, especially in the elderly. Therefore, influenza vaccination is related to the prevention of pneumonia. The report from the United States Renal Data System (USRDS) in 2007 showed that influenza vaccination for CKD patients aged over 66 years decreased total mortality and hospitalization rates from January to March compared to that of unvaccinated patients.

Pneumonia is the 4th leading cause of death in patients aged over 65 years in Japan, and $95 \%$ of deaths from pneumonia occur in patients aged over 65 years. Pneumococcus is the most common pathogen in communityacquired pneumonia of the elderly, and it is reported that $30-50 \%$ of Pneumococcus is drug-resistant.

Viasus et al. performed an observational analysis of a prospective cohort of hospitalized adults with pneumonia and showed that Pneumococcal vaccination was effective for preventing moderate and severe pneumonia in patients with CKD.

The Center for Disease Control and Prevention (CDC) recommends Pneumococcal vaccination for all patients aged over 65 years, and for high-risk patients aged from 2 to 65 years (chronic heart disease, chronic lung disease and diabetes mellitus). The CDC also recommends vaccination for patients with CKD and nephrotic syndrome, but the recommendation level is low.

Fuchshuber et al. reported that the antibody levels of the Pneumococcal vaccine should be monitored in CKD patients considering an observed rapid decline in as early as 6 months after vaccination. The CDC recommends revaccination for patients over 65 years of age if 5 years have passed from the previous vaccination. CKD patients have a decreased capacity to maintain the antibody, and therefore, have the potential to lose immunity faster 
compared to healthy patients. In summary, CKD patients need to be more closely monitored.

\section{Bibliography}

1. Collins AJ, et al. Excerpts from the United States Renal Data System 2007 annual data report. Am J Kidney Dis. 2008;51:S1-320.

2. Viasus D, et al. Nephrol Dial Transplant. 2011;26:2899-906. (Level 4)

3. Fuchshuber A, et al. Nephrol Dial Transplant. 1996;11:468-73. (Level 4)

CQ7 Does hyperuricemia affect the onset and progression of $\mathrm{CKD}$ ?

Hyperuricemia can affect the development of CKD

Hyperuricemia and renal dysfunction are co-related. Hyperuricemia causes renal dysfunction and renal dysfunction causes hyperuricemia due to low excretion of uric acid from the kidney. A recent report showed that hyperuricemia itself causes renal vascular injury and interstitial damage without deposition of uric acid in the kidney. This suggests that hyperuricemia can affect the onset and progression of CKD.

Iseki et al. reported that hyperuricemia was associated with a higher incidence of ESRD and was an independent predictor of ESRD in women in a Japanese cohort study. Bellomo et al. showed that elevated serum uric acid levels were associated with a greater likelihood of a decrease in eGFR, and serum uric acid level was an independent risk factor for decreased kidney function in a prospective observational cohort study. However, Chonchol et al. concluded that no significant association was found between the uric acid level and incident CKD in the Cardiovascular Health Study.

Obermayr et al. reported that elevated levels of uric acid independently increased the risk for new-onset kidney disease. Kawashima et al. showed that asymptomatic hyperuricemia is a predictive factor for new-onset CKD for Japanese male workers.

Madero et al. reported that in patients with CKD stages G3 and G4, hyperuricemia appeared to be an independent risk factor for all-cause and CVD-related mortality, but not for kidney failure.

Above all, current epidemiological studies show that hyperuricemia is strongly related to the onset and progression of renal injury and CKD.

\section{Bibliography}

1. Iseki K, et al. Am J Kidney Dis. 2004;44:642-50. (Level 4)

2. Bellomo G, et al. Am J Kidney Dis. 2010;56:264-72. (Level 4)
3. Chonchol M, et al. Am J Kidney Dis. 2007;50:239-47. (Level 4)

4. Obermayr RP, et al. J Am Soc Nephrol. 2008;19:2407-13. (Level 4)

5. Kawashima M, et al. BMC Nephrol. 2011;12:31-7. (Level 4)

6. Madero M, et al. Am J Kidney Dis. 2009;53:796-803. (Level 4)

CQ8 Is therapy for hyperuricemia recommended to prevent the development of CKD?

We suggest therapy for hyperuricemia for preventing the development of CKD. (Grade C1)

A therapeutic interventional study on hyperuricemia is the best way to demonstrate the role of hyperuricemia in CKD. However, so far, evidence for the efficacy of therapeutic intervention is inconclusive.

Siu et al. reported that the treatment of hyperuricemia affected the development of CKD. They conducted a prospective, randomized, controlled trial on 54 hyperuricemic patients with CKD. Patients were randomly assigned to treatment with allopurinol, $100-300 \mathrm{mg} / \mathrm{d}$, or to continuing their usual therapy for 12 months as the control group. Serum uric acid levels were significantly decreased in subjects treated with allopurinol. There was a trend toward a lower serum creatinine level in the treatment group compared to the controls after 12 months of therapy, although the difference was not statistically significant. The study concluded that allopurinol therapy significantly decreased serum uric acid levels in hyperuricemic patients with mild to moderate chronic kidney disease. Its use was safe and helped to preserve kidney function during the 12 months of therapy compared to the controls.

Goicoechea et al. conducted a prospective, randomized trial of 113 patients with eGFR $<60 \mathrm{ml} / \mathrm{min}$. Patients were randomly assigned to treatment with allopurinol $100 \mathrm{mg} / \mathrm{day}$ ( $\mathrm{n}=57)$ or to continuing their usual therapy $(\mathrm{n}=56)$ for 24 months. Serum uric acid and C-reactive protein (CRP) levels were significantly decreased in the subjects treated with allopurinol. Allopurinol treatment slowed down renal disease progression independently of age, gender, diabetes, CRP, albuminuria, and the use of renin-angiotensin system blockers. Allopurinol treatment reduced the risk of cardiovascular events by $71 \%$ compared to standard therapy.

Kanbay et al. conducted a prospective study to investigate the benefits of allopurinol treatment in hyperuricemic patients with normal renal function. Forty-eight hyperuricemic and 21 normouricemic patients were included in the study. Hyperuricemic patients received $300 \mathrm{mg} /$ day allopurinol for 3 months. In the allopurinol group, serum uric acid levels, GFR, systolic and diastolic blood pressure, and CRP levels significantly improved. Management of hyperuricemia may prevent the progression of renal 
disease, even in patients with normal renal function, suggesting that early treatment with allopurinol should be an important part of the management of CKD patients.

Above all, management of hyperuricemia is effective for preventing the progression of renal dysfunction in CKD. Allopurinol is a xanthine oxidase inhibitor and has the potential to reduce oxidative stress. Therefore a clinical study on allopurinol treatment investigating effects attributable to a mechanism other than decreasing uric acid levels is necessary. Results of these studies need to be confirmed with an additional prospective trial involving a larger cohort of patients to determine the long-term efficacy of hyperuricemic therapy and relevance to specific CKD subpopulations.

Pain control in hyperuricemic therapy is also important. Several classes of anti-inflammatory agents are effective for the treatment of acute gout, including nonsteroidal antiinflammatory agents (NSAIDs), colchicine and glucocorticoids. In general, NSAIDs are frequently used as the initial therapy for acute gout, but NSAIDs may cause renal injury. Gruff et al. reported that a short course of oral corticosteroid therapy can be used effectively for acute gout when NSAIDs are contraindicated. The use of prednisone $30-50 \mathrm{mg}$ or its equivalent initially, which was then tapered gradually over 10 days, resulted in clinical resolution without rebound arthropathy or steroid complications in most patients.

Colchicine is used in patients with NSAIDs intolerance or with an absolute (or often relative) contraindication. Colchicine is most likely to be effective if the treatment is started within 12-24 h of symptom onset. However, colchicine is contraindicated in patients with advanced renal or hepatic impairment because both the kidneys and liver participate in colchicine metabolism. Long-term colchicine treatment in patients with milder renal or hepatic impairment in combination with CYP3A4 inhibitors (e.g. clarithromycin) has been associated with a greater risk for colchicine toxicity due to the resulting increased serum concentration of colchicines.

Febuxostat is a new drug for hyperuricemia that received marketing approval by the European Medicines Agency on April 21, 2008 and was approved by the US Food and Drug Administration on February 16, 2009. Febuxostat is a xanthine oxidase inhibitor like allopurinol and is used in patients with mild-to-moderate renal impairment. Efficacy for all CKD stages should be further investigated in a large cohort study.

\section{Bibliography}

1. Groff GD, et al. Systemic steroid therapy for acute gout: a clinical trial and review of the literature. Semin Arthritis Rheum. 1990;19:329-36.

2. Siu YP, et al. Am J Kidney Dis. 2006;47:51-9. (Level 2)

3. Goicoechea M, et al. Clin J Am Soc Nephrol. 2010;5:1388-93. (Level 2)
4. Kanbay M, et al. Int Urol Nephrol. 2007;39:1227-33. (Level 4)

5. Hung IF, et al. Clin Infect Dis. 2005;41:291-300. (Level 4)

\section{Chapter 3: CKD and Nutrition}

CQ1 Is dietary protein restriction recommended to prevent the progression of CKD?

We recommend individualized protein restriction to patients with CKD in accordance with their specific clinical condition. (Grade B)

Protein restriction in advanced CKD mitigates the burden of uremic toxins, acid, and phosphate and may decrease intraglomerular pressure. Accordingly, protein restriction delays the development of end-stage renal disease that requires renal replacement therapy, although it does not significantly slow the rate of renal function decline. As the standard of care for stage G3b-5 CKD, we recommend that patients be encouraged to lower their dietary protein intake to $0.6-0.8 \mathrm{~g} / \mathrm{kg}$. standard body weight (SBW)/day. Actual protein intake should be estimated by analyzing the urea content in the 24-h urine sample using the Maroni formula; it should then be evaluated by comparing it with the results of previously published studies, which showed that the achieved protein intakes were $0.75-0.9 \mathrm{~g} / \mathrm{kg} /$ day in clinical trials with protein restriction of $0.6-0.8 \mathrm{~g} / \mathrm{kg} /$ day. Several studies also demonstrated both the efficacy and potential hazards of a very low protein diet. Therefore, the potential benefits and risks of severe protein restriction should be specifically assessed for each patient. Digestibility and the amino acid score of protein sources should be taken into consideration when prescribing protein restriction diets. For early CKD with the risk of progression, we suggest encouraging patients to lower their protein intake to $0.8-1.0 \mathrm{~g} / \mathrm{kg} \cdot \mathrm{SBW} /$ day. The extent of protein restriction should be individualized in accordance with each patient's specific clinical condition, including severity, risk of progression, nutritional status, and adherence.

\section{Bibliography}

1. Pan Y, et al. Am J Clin Nutr. 2008;88:660-6. (Level 1)

2. Gansevoort RT, et al. Nephrol Dial Transplant. 1995;10:497-504. (Level 3)

3. Williams PS, et al. Q J Med. 1991;81:837-55. (Level 2)

4. D'Amico G, et al. Nephrol Dial Transplant. 1994;9:1590-4. (Level 2)

5. Mircescu G, et al. J Ren Nutr. 2007;17:179-88. (Level 2)

6. Rosman JB, et al. Kidney Int Suppl. 1989;27:S96-102. (Level 2) 
7. Cianciaruso B, et al. Am J Kidney Dis. 2009;54:1052-61. (Level 2)

8. Fouque D, et al. Cochrane Database Syst Rev. 2009:CD001892. (Level 1)

9. Pedrini MT, et al. Ann Intern Med. 1996;124:627-32. (Level 1)

10. Hansen HP, et al. Kidney Int. 2002;62:220-8. (Level 2)

11. Kasiske BL, et al. Am J Kidney Dis. 1998;31:954-61. (Level 1)

12. Robertson L, et al. Cochrane Database Syst Rev. 2007;CD002181. (Level 1)

13. Koya D, et al. Diabetologia. 2009;52:2037-45. (Level 2)

14. Feiten SF, et al. Eur J Clin Nutr. 2005;59:129-36. (Level 2)

15. Jungers P, et al. Kidney Int. 1987;22(Suppl):S67-71. (Level 2)

16. Cianciaruso B, et al. Nephrol Dial Transplant. 2008;23:636-44. (Level 2)

17. Malvy D, et al. J Am Coll Nutr. 1999;18:481-6. (Level 2)

18. Di Iorio BR, et al. Kidney Int. 2003;64:1822-8. (Level 2)

19. Ihle BU, et al. N Engl J Med. 1989;321:1773-7. (Level 2)

20. Levey AS, et al. Am J Kidney Dis. 1996;27:652-63. (Level 4)

21. Zeller K, et al. N Engl J Med. 1991;324:78-84. (Level 2)

22. Klahr S, et al. J Am Soc Nephrol. 1995;5:2037-47. (Level 3)

23. Walser M, et al. Am J Kidney Dis. 1996;28:354-64. (Level 5)

24. Chauveau P, et al. J Ren Nutr. 2007;17:250-7. (Level 5)

25. Aparicio M, et al. J Am Soc Nephrol. 2000;11:708-16. (Level 5)

26. Brunori G, et al. Am J Kidney Dis. 2007;49:569-80. (Level 2)

27. Menon V, et al. Am J Kidney Dis. 2009;53:208-17. (Level 3)

28. Klahr S, et al. N Engl J Med. 1994;330:877-84. (Level 2)

29. Coresh J, et al. J Am Soc Nephrol. 1995;6:1379-85. (Level 5)

30. Chauveau P, et al. Am J Clin Nutr. 2009;90:969-74. (Level 5)

31. Ideura T, et al. Am J Kidney Dis. 2003;41:S31-4. (Level 4)

32. Ideura T, et al. Contrib Nephrol. 2007;155:40-9. (Level 4)

CQ2 Does dietary salt restriction reduce the risk of progression of CKD to ESRD, cardiovascular disease and mortality?

We recommend restriction of salt intake to less than $6 \mathrm{~g} /$ day to lower the amount of urinary protein and the risk of ESRD, CVD and mortality. (Grade B) We do not recommend restriction of salt intake to less than $3 \mathrm{~g}$ /day because it may increase the risk of ESRD and mortality. (Grade C2)

High salt intake increases blood pressure and urinary protein, which are risk factors for CKD progression to endstage renal disease (ESRD) as well as the development of cardiovascular disease (CVD) and mortality. Small RCTs have demonstrated that salt restriction (to approximately less than $6 \mathrm{~g} /$ day) reduces blood pressure and urinary protein, and may enhance the antiproteinuric effects of renin-angiotensin inhibitors. Therefore, we recommend restricting salt intake to less than $6 \mathrm{~g} /$ day in patients with CKD. However, many patients do not achieve this level of salt restriction. The Dietary Reference Intake for the
Japanese 2010 suggests that the tentative target is less than 9 and $7.5 \mathrm{~g} /$ day for males and females, respectively, and these targets may be feasible for stage G1-2 CKD patients. It should also be noted that several large observational studies have shown a J-shaped association of urinary sodium excretion with the incidence of cardiovascular disease and mortality in patients with established CVD and diabetes mellitus. In type 1 diabetic patients, individuals with the highest and the lowest daily urinary sodium excretion had reduced cumulative survival. Moreover, individuals with the lowest urinary sodium excretion (approximately less than $50 \mathrm{mmol} /$ day) had the highest cumulative incidence of ESRD. Since some advanced CKD patients with salt-losing nephropathy may have a high obligatory salt loss, extreme salt restriction may be harmful for these patients. Therefore, we do not recommend the restriction of salt intake to less than $3 \mathrm{~g} /$ day. Estimating the daily sodium intake from a $24-\mathrm{h}$ urine collection is clinically useful to give an indication of the excess amount of sodium intake and to monitor patient adherence.

\section{Bibliography}

1. Thomas MC, et al. Diabetes Care. 2011;34:861-6. (Level 4)

2. Yu W, et al. Int Urol Nephrol (Epub 2011 May 21). (Level 4)

3. Slagman MC, et al. BMJ. 2011;343:d4366. (Level 2)

4. Vogt L, et al. J Am Soc Nephrol. 2008;19:999-1007. (Level 2)

5. Lambers Heerspink HJ, et al. Kidney Int. 2012;82:330-7. (Level 4)

6. Verhave JC, et al. J Intern Med. 2004;256:324-30. (Level 5)

7. Vedovato M, et al. Diabetologia. 2004;47:300-3. (Level 2)

8. He FJ, et al. Hypertension. 2009;54:482-8. (Level 2)

9. Lin J, et al. Clin J Am Soc Nephrol. 2010;5:836-43. (Level 4)

10. Vegter S, et al. J Am Soc Nephrol (Epub 2011 Dec 1). (Level 4)

11. Strazzullo P, et al. BMJ. 2009;339:b4567. (Level 4)

12. Stolarz-Skrzypek K, et al. JAMA. 2011;305:1777-85. (Level 4)

13. O’Donnell MJ, et al. JAMA. 2011;306:2229-38. (Level 4)

14. Taylor RS, et al. Cochrane Database Syst Rev. 2011;CD009217. (Level 1)

15. Ekinci EI, et al. Diabetes Care. 2011;34:703-9. (Level 4)

16. Kutlugün AA, et al. Nephron Clin Pract. 2011;118:c361-6. (Level 5)

17. Imai E, et al. Clin Exp Nephrol. 2011;15:861-7. (Level 5)

CQ3 What should the target range of serum potassium levels be in CKD?

We suggest that serum potassium levels should be maintained in the range between 4.0 and $5.4 \mathrm{mEq} / \mathrm{L}$ in patients with CKD. (Grade C1)

Patients with advanced CKD are at risk of hyperkalemia. Other risk factors for hyperkalemia include metabolic 
acidosis, diabetes, congestive heart failure, advanced age, and the use of $\beta$ blockers and renin-angiotensin-aldosterone system (RAAS) inhibitors. In a retrospective cohort of patients cared for over a single year in the Veterans Health Administration, hyperkalemia ( $\geq 5.5 \mathrm{mEq} / \mathrm{L})$ was associated with high mortality. Other prospective cohort studies have demonstrated that patients with hypokalemia $(<4.0 \mathrm{mEq} / \mathrm{L})$ also were at high risk of all-cause mortality, cardiovascular mortality, heart failure, and end-stage renal disease. Accordingly, we suggest that serum potassium levels should be maintained between 4.0 and $5.4 \mathrm{mEq} / \mathrm{L}$ in patients with CKD. In patients with $\mathrm{CKD}$ and hyperkalemia, metabolic acidosis should be evaluated and corrected appropriately. When serum potassium levels exceed $5.5 \mathrm{mEq} / \mathrm{L}$ without metabolic acidosis, nutritional advice relating to fruit, vegetable, and protein intake should be provided. Other treatment options such as reducing the RAAS inhibitor dosage and administering potassium absorbing resin can also be pursued. For hypokalemia $(\mathrm{K}<4.0 \mathrm{mEq} / \mathrm{L})$, the administration of potassium-lowering drugs such as diuretics and the dietary intake of fruits, vegetables, and protein sources should be evaluated and managed.

\section{Bibliography}

1. Einhorn LM, et al. Arch Intern Med. 2009;169:1156-6. (Level 4)

2. Miao Y, et al. Diabetologia. 2011;54:44-50. (Level 4)

3. ONTARGET Investigators. N Engl J Med. 2008;358:1547-59. (Level 2)

4. Korgaonkar S, et al. Clin J Am Soc Nephrol. 2010;5:762-9. (Level 4)

5. Bowling CB, et al. Circ Heart Fail. 2010;3:253-60. (Level 4)

CQ4 Should metabolic acidosis be corrected to prevent the progression of CKD and the reduction of mortality?

We recommend normalizing serum bicarbonate level in patients with CKD and metabolic acidosis to slow the rate of renal function decline and reduce the risk of ESRD and mortality. (Grade B)

Metabolic acidosis, frequently observed in patients with advanced $\mathrm{CKD}$, increases the degradation of muscle protein, reduces albumin synthesis and leads to abnormal bone metabolism. Observational studies have shown that a low serum bicarbonate level is associated with a rapid renal function decline and a high risk of both ESRD and mortality, and that a high serum bicarbonate level is also associated with high mortality. Several RCTs have revealed that sodium bicarbonate delays the development of ESRD and improves the nutritional status of patients with advanced CKD and metabolic acidosis. Additionally, alkali therapy with sodium bicarbonate or sodium acetate slows the rate of renal function decline not only in patients with advanced CKD and metabolic acidosis, but also in those at stage G2 CKD with normal serum bicarbonate levels. Based on the final serum bicarbonate levels in intervention groups, we recommend that the serum bicarbonate level should be maintained at least above $22 \mathrm{mEq} /$ L. However, overcorrection of metabolic acidosis by alkali therapy should be avoided.

\section{Bibliography}

1. Shah SN, et al. Am J Kidney Dis. 2009;54:270-7. (Level 4)

2. Menon V, et al. Am J Kidney Dis. 2010;56:907-14. (Level 4)

3. Raphael KL, et al. Kidney Int. 2011;79:356-62. (Level 4)

4. Kovesdy CP, et al. Nephrol Dial Transplant. 2009;24:1232-7. (Level 4)

5. Navaneethan SD, et al. Clin J Am Soc Nephrol. 2011;6:2395-402. (Level 4)

6. de Brito-Ashurst I, et al. J Am Soc Nephrol. 2009;20:2075-84. (Level 2)

7. Disthabanchong S, et al. Am J Nephrol. 2010;32:549-56. (Level 2)

8. Phisitkul S, et al. Kidney Int. 2010;77:617-23. (Level 4)

9. Mahajan A, et al. Kidney Int. 2010;78:303-9. (Level 2)

10. Goraya N, et al. Kidney Int. 2012;81:86-93. (Level 2)

CQ5 What should the target range of serum phosphate levels be in $\mathrm{CKD}$ ?

We suggest maintaining serum phosphate levels within the normal range. (Grade $\mathrm{C} 1$ )

Serum phosphate levels increase as renal function declines, but remain within the normal range in moderate CKD due to elevated levels of the phosphaturic hormones (FGF23 and parathyroid hormone). However, several population studies have revealed that serum phosphate levels, even in the normal range, are positively associated with mortality, cardiovascular disease, the progression of $\mathrm{CKD}$, and end-stage renal disease, and that these relationships are pronounced in diabetic patients. Furthermore, a sub-analysis of the REIN study indicated that hyperphosphatemia may diminish the renoprotective effect of angiotensin converting enzyme inhibitor (ramipril) in patients with non-diabetic CKD. Therefore, we suggest maintaining serum phosphate levels within the normal range. Consumption of proteins and foods with a high phosphorus-protein ratio should be avoided by patients with CKD and hyperphosphatemia to restrict their phosphate intake. Additionally, it should be noted that most food labels do not display the phosphorous content although the use of phosphate additives is increasing in Japan. Several fast food products, processed food products, and instant meals are rich in phosphate-containing 
additives. Thus, patient education about avoiding phosphate-containing additives may reduce the phosphate burden. However, future studies are required to determine the timing and indices of phosphate restriction in CKD patients at the risk of progression.

\section{Bibliography}

1. Bellasi A, et al. Clin J Am Soc Nephrol. 2011;6:883-91. (Level 4)

2. Voormolen N, et al. Nephrol Dial Transplant. 2007;22:2909-16. (Level 4)

3. Kestenbaum B, et al. J Am Soc Nephrol. 2005;16:520-8. (Level 4)

4. Eddington H, et al. Clin J Am Soc Nephrol. 2010;5:2251-7. (Level 4)

5. Kovesdy CP, et al. Clin Nephrol. 2010;73:268-75. (Level 4)

6. Connolly GM, et al. Transplantation. 2009;87:1040-4. (Level 4)

7. Moore J, et al. Clin Transplant. 2011;25:406-16. (Level 4)

8. Tonelli M, et al. Circulation. 2005;112:2627-33. (Level 4)

9. Abramowitz M, et al. Clin J Am Soc Nephrol. 2010;5:1064-71. (Level 4)

10. Dhingra R, et al. Arch Intern Med. 2007;167:879-85. (Level 4)

11. Larsson TE, et al. Arterioscler Thromb Vasc Biol. 2010;30:333-9. (Level 4)

12. Menon V, et al. Am J Kidney Dis. 2005;46:455-63. (Level 4)

13. Murtaugh MA, et al. Nephrol Dial Transplant. 2012;27:990-6. (Level 4)

14. Smith DH, et al. Nephrol Dial Transplant. 2010;25:166-74. (Level 4)

15. Schwarz S, et al. Clin J Am Soc Nephrol. 2006;1:825-31. (Level 4)

16. Zoccali C, et al. J Am Soc Nephrol. 2011;22:1923-30. (Level 4)

17. O'Seaghdha CM, et al. Nephrol Dial Transplant. 2011;26:2885-90. (Level 4)

18. Chue CD, et al. Nephrol Dial Transplant. 2011;26:2576-82. (Level 4)

19. Sullivan C, et al. JAMA. 2009;301:629-35. (Level 2)

20. Moe SM, et al. Clin J Am Soc Nephrol. 2011;6:257-64. (Level 3)

\section{Chapter 4: Hypertension and CVD in CKD}

CQ1 Does hypertension cause or aggravate CKD?

There is a strong association between CKD and hypertension whereby each can cause or aggravate the other.

Hypertension causes CKD and exacerbates its clinical condition. Inversely, CKD causes hypertension and is a risk factor that can aggravate hypertension. In the MRFIT study and prospective cohort studies, hypertension was found to be a significant risk factor for end-stage kidney disease (ESKD) regardless of gender. When the systolic blood pressure (BP) was elevated by $10 \mathrm{mmHg}$, the onset of ESKD was increased by $20-30 \%$. In addition, while the 10 -year hazard ratio (HR) for the occurrence of G1 or G2 category of CKD is 1.21-1.67 with grade I hypertension (JSH2009), it increases to 1.73-2.17 with grade II-III hypertension.

In addition, in an observational study of patients with hypertension without CKD, the renal function deteriorated in patients with inadequate lowering of their blood pressure. Furthermore, it is important to diagnose hypertension at an early phase and to start appropriate anti-hypertensive therapy to prevent the progression of CKD to ESKD.

\section{Bibliography}

1. Klag MJ, et al. N Engl J Med. 1996;334:13-8. (Level 4)

2. Klag MJ, et al. JAMA 1997;277:1293-8. (Level 4)

3. Reynolds K, et al. J Am Soc Nephrol. 2007;18:1928-35. (Level 4)

4. Tozawa M, et al. Hypertension. 2003;41:1341-5. (Level 4)

5. Yamagata K, et al. Kidney Int. 2007;71:159-66. (Level 4)

6. The Centers for Disease Control and Prevention Chronic Kidney Disease Surveillance Team. Hypertension. 2010;55:1102-9. (Level 4)

7. Vupputuri S, et al. Hypertension. 2003;42:1144-9. (Level 4)

8. Yano Y, et al. Kidney Int. 2012;81:293-9. (Level 4)

CQ2 Is anti-hypertensive therapy recommended for the management of CKD? (Fig. 1)

We strongly recommend anti-hypertensive therapy in CKD to inhibit or prevent the progression of renal dysfunction and to prevent the occurrence or recurrence of CVD. (Grade A)

In all diabetic patients with $\mathrm{CKD}$, we recommend the target level of clinic BP $<130 / 80 \mathrm{mmHg}$, irrespective of the presence or absence of albuminuria/proteinuria. (Grade B)

In all non-diabetic patients with CKD, we strongly recommend the target level of clinic $\mathrm{BP}<140$ / $90 \mathrm{mmHg}$ at all times, irrespective of the presence or absence of albuminuria/proteinuria. (Grade A) In non-diabetic patients with $\mathrm{CKD}$ of $\mathrm{A} 2$ or $\mathrm{A} 3$ category, we suggest the target level of clinic BP $<130 / 80 \mathrm{mmHg}$. (Grade C1)

1. Recommendation of anti-hypertensive therapy

The aim of anti-hypertensive therapy is to inhibit the progression of CKD and to decrease the occurrence of CVD and mortality. Since many previous studies showed that the rate of eGFR decrease is slowed according to the BP levels achieved during anti-hypertensive therapy, it is clear that anti-hypertensive therapy is effective for inhibiting the progression of CKD. It is also clear that 
antihypertensive therapy with $\mathrm{BP}$ reduction to less than $140 / 90 \mathrm{mmHg}$ is beneficial and recommended to decrease the risks of CVD and mortality. On the other hand, the benefits of further strict $\mathrm{BP}$ reduction to less than 130/80 $\mathrm{mmHg}$ have not been established, particularly in non-diabetic CKD.

2. Antihypertensive therapy for suppressing the progression of CKD and the occurrence of CVD in diabetic CKD

The results of a recent meta-analysis examining the optimal BP target in subjects with diabetes or those with IGT suggest that in patients with diabetes or IGT, a target BP of $130-135 \mathrm{mmHg}$ is acceptable. However, with more aggressive clinic BP goals $(<130 \mathrm{mmHg}$ ), target organ heterogeneity was observed in that the risk of stroke continued to fall, but there was no benefit in terms of the risk of other macrovascular or microvascular (cardiac, renal and retinal) events, and the risk of serious adverse events even increased. Despite these risks, since the suppression of stroke in diabetic CKD is an important issue in Japan, we recommend the target level of clinic BP to be $<130 / 80 \mathrm{mmHg}$, irrespective of the presence or absence of albuminuria/proteinuria (Grade B).

3. Antihypertensive therapy for suppressing the progression of CKD and the occurrence of CVD in non-diabetic CKD

In all non-diabetic CKD, we strongly recommend the target level of clinic BP to be maintained consistently at $<140$ / $90 \mathrm{mmHg}$, irrespective of the presence or absence of albuminuria/proteinuria (Grade A). However, the rationale for further intensive BP reduction to less than 130/80 $\mathrm{mmHg}$ in all CKD, irrespective of the presence or absence of albuminuria/ proteinuria, cannot be established. In a recent systematic analysis of 3 RCT phases of the MDRD, REIN-2 and AASK studies and 2 extension cohort phases of the MDRD and AASK studies, a better prognosis was found for renal events in the intensive BP control group (target clinic BP level: less than 125-130/75-80 $\mathrm{mmHg}$ ) compared with the standard BP control group (target clinical BP level: less than 140/90 $\mathrm{mmHg}$ ) in non-diabetic $\mathrm{CKD}$ with proteinuria. However, since these results regarding the relationship between $\mathrm{BP}$ levels and the suppression of CVD occurrence in non-diabetic CKD are essentially derived from observational studies and sub-analyses of RCTs without high-level evidence, justification for intensive $\mathrm{BP}$ reduction to less than 130/80 $\mathrm{mmHg}$ to suppress CVD, particularly stroke, in CKD, needs further accumulation of "high-level" evidence. Therefore, in non-diabetic patients with category $\mathrm{A} 2$ and $\mathrm{A} 3 \mathrm{CKD}$, we can only tentatively suggest the target level of clinic BP to be $<130 / 80 \mathrm{mmHg}$ (Grade C1).

4. Emerging importance of tailoring BP treatment in CKD

Recent evidence has suggested that tailoring BP treatment according to age, circadian BP rhythm and seasonal BP change may be important for the management of CKD. For example, in middle-aged CKD with chronic glomerulonephritis, RAS inhibitors (ARB, ACEI) are recommended as the first-line anti-hypertensive drugs. The dosage of RAS inhibitors may be cautiously titrated to reduce proteinuria to the levels of A1 or A2 categories, with attention to the symptoms of hypotension and decline of eGFR. In addition, it has been reported that seasonal BP changes may affect conditions of hypertension and CKD. Particularly tailoring anti-hypertensive therapy is suggested to be crucial for the management of CKD in elderly patients.

\section{Bibliography}

1. Sleigh P, et al. J Hypertens. 2009;27:1360-9. (Level 2)

2. Bakris GL, et al. Am J Kidney Dis. 2000;36:646-61. (Level 4)

3. Jafar TH, et al. Ann Intern Med. 2003;139:244-52. (Level 4)

4. Adler AI, et al. BMJ. 2000;321:412-9. (Level 4)

5. ADVANCE Collaborative Group. J Am Soc Nephrol. 2009;20:883-92. (Level 2)

6. Uzu T, et al. J Am Soc Hypertens. 2012;6:124-31. (Level 4)

7. Cushman WC, et al. N Engl J Med. 2010;362:1575-85. (Level 2)

8. Bangalore S, et al. Circulation. 2011;123:2799-810. (Level 1)

9. Pohl MA, et al. J Am Soc Nephrol. 2005;16:3027-37. (Level 2)

10. Cooper-DeHoff RM, et al. JAMA. 2010;304:61-8. (Level 2)

11. Kawamori R, et al. Diabetes Res Clin Pract. 2009;83:241-8. (Level 4)

12. Klahr S, et al. N Engl J Med. 1994;330:877-84. (Level 2)

13. Wright JT Jr, et al. JAMA. 2002;288:2421-31. (Level 2)

14. Ruggenenti P, et al. Lancet. 2005;365:939-46. (Level 2)

15. Peralta CA, et al. Arch Intern Med. 2012;172:41-7. (Level 4)

16. Peterson JC, et al. Ann Intern Med. 1995;123:754-62. (Level 2)

17. Sarnak MJ, et al. Ann Intern Med. 2005;142:342-51. (Level 2)

18. Appel LJ, et al. N Engl J Med. 2010;363:918-29. (Level 4)

19. Upadhyay A, et al. Ann Intern Med. 2011;154:541-8. (Level 4)

20. Ninomiya T, et al. Circulation. 2008;118:2694-701. (Level 4)

21. Irie F, et al. Kidney Int. 2006;69:1264-71. (Level 4)

22. Kokubo Y, et al. Stroke. 2009;40:2674-9. (Level 4)

23. Lawes CM, et al. J Hypertens. 2003;21:707-16. (Level 4)

24. Weiner DE, et al. J Am Soc Nephrol. 2007;18:960-6. (Level 4)

25. Ninomiya T, et al. Kidney Int. 2008;73:963-70. (Level 2)

CQ3 Is restriction of salt intake recommended for the management of hypertension in CKD?

We strongly recommend restriction of salt intake to $<6 \mathrm{~g}$ /day to inhibit the progression of renal dysfunction. (Grade B)

We do not suggest more strict restriction of salt intake to $<3 \mathrm{~g} /$ day due to the possibility of increasing the mortality or accelerating the progression of renal dysfunction. (Grade $\mathrm{C} 2$ )

The salt restriction reportedly reduced proteinuria and inhibited the progression of CKD. The dietary sodium 


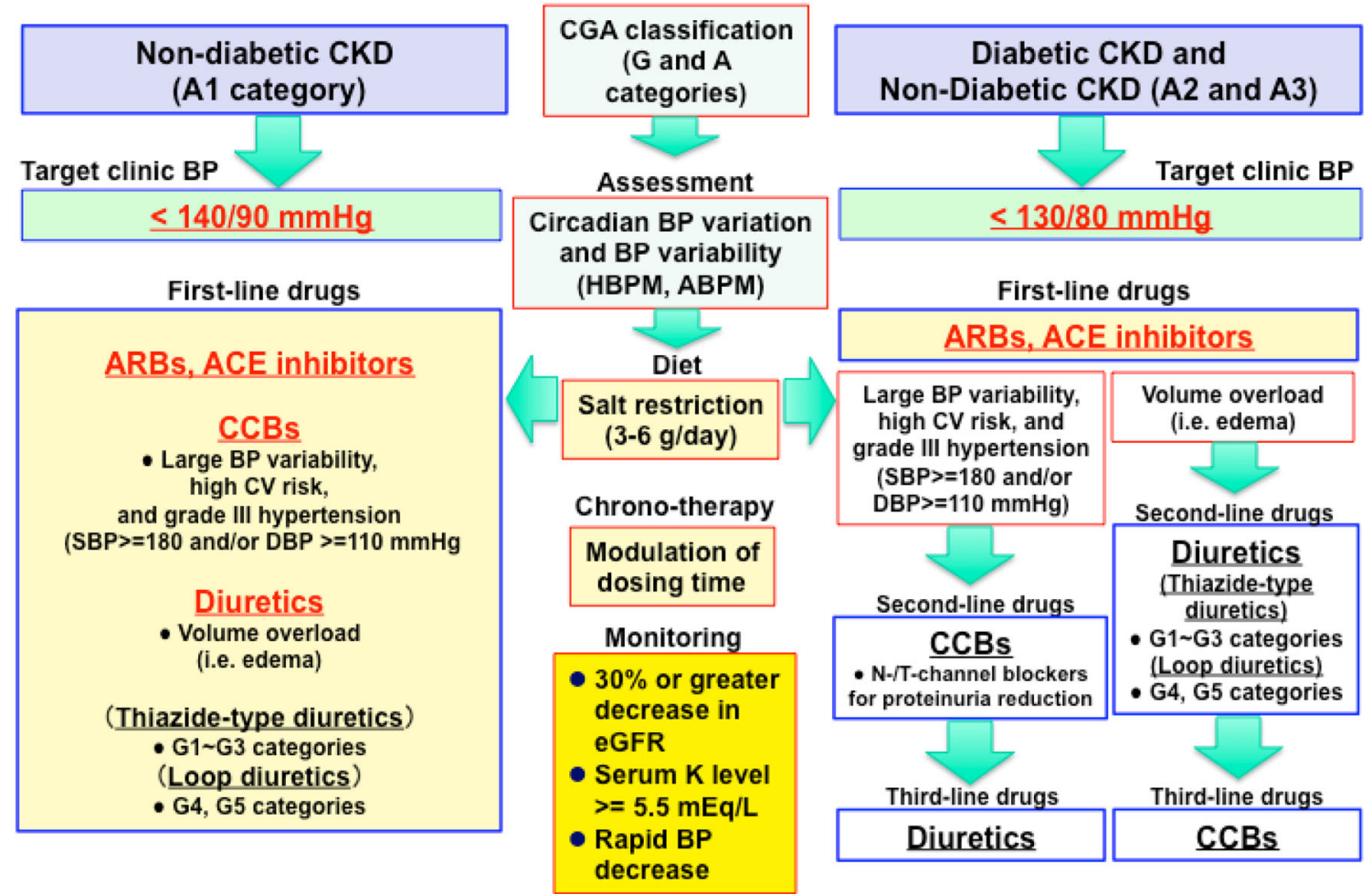

Fig. 1 Summary of the recommended management of hypertension with CKD

restriction to $<6 \mathrm{~g} /$ day was more effective than dual RAS inhibition for reducing proteinuria and $\mathrm{BP}$ in non-diabetic CKD. In addition, therapeutic effects of ARB compared with non-RAS inhibitor-based therapy on renal and cardiovascular outcomes were greater in diabetic CKD with lower rather than higher dietary sodium intake.

Collectively, we recommend salt restriction to inhibit the progression of CKD via efficient BP reduction. The recommended target level of salt intake is 3-6 g/day. On the other hand, it is reported that excessive salt restriction may cause appetite loss and promote renal dysfunction due to dehydration, especially in elderly CKD. In CKD with type 1 diabetes, salt intake was independently associated with overall mortality and ESRD, and there was a significant increase in mortality in subjects with urinary sodium excretion =/ $<50 \mathrm{mmol}$ (salt intake $=/<3 \mathrm{~g} /$ day). Therefore, we do not suggest further reduction of salt intake to $<3 \mathrm{~g}$ /day due to the possibility of increasing the mortality and accelerating the progression of renal dysfunction (Grade C2). When salt restriction is difficult, we recommend administration of lowdose diuretics. Thiazide or thiazide-like diuretics in the G1, G2 or G3 categories and loop diuretics in the G4 or G5 categories are beneficial for promoting sodium excretion in CKD.

\section{Bibliography}

1. Sacks FM, et al. N Engl J Med. 2001;344:3-10. (Level 2)

2. Swift PA, et al. Hypertension. 2005;46:308-12. (Level 2)
3. Cianciaruso B, et al. Miner Electrolyte Metab. 1998;24:296-301. (Level 4)

4. HONEST (HOlland NEephrology STudy) Group. BMJ. 2011;343:d4366. (Level 2)

5. Vegter S, et al. J Am Soc Nephrol. 2012;23:165-73. (Level 4)

6. Lambers Heerspink HJ, et al. Kidney Int. 2012;82:330-7. (Level 4)

7. Stolarz-Skrzypek K, et al. JAMA. 2011;305:1777-85. (Level 4)

8. Thomas MC, et al. Diabetes Care. 2011;34:861-6. (Level 4)

CQ4 What kind of anti-hypertensive drugs are recommended as the first line medication for the management of hypertension in CKD? (Fig. 1)

In diabetic $\mathrm{A} 1$ category $\mathrm{CKD}$, we tentatively suggest RAS inhibitors (ACE inhibitors or ARBs) as the firstline anti-hypertensive drugs. (Grade $\mathrm{C} 1$ )

In diabetic $\mathrm{A} 2$ or $\mathrm{A} 3$ category $\mathrm{CKD}$, we strongly recommend RAS inhibitors as the first-line antihypertensive drugs. (Grade A)

In non-diabetic A1 category $\mathrm{CKD}$, we recommend RAS inhibitors, CCB or diuretics as the first-line antihypertensive drugs. (Grade B)

In non-diabetic $\mathrm{A} 2$ or $\mathrm{A} 3$ category $\mathrm{CKD}$, we recommend RAS inhibitors as the first-line antihypertensive drugs. (Grade B) 
1. First-line anti-hypertensive drugs for diabetic CKD

In diabetic $\mathrm{A} 2$ and $\mathrm{A} 3$ category $\mathrm{CKD}$, we recommend RAS inhibitors as first-line anti-hypertensive drugs. The renal and cardiovascular protective effects of RAS inhibition depend on the degree of albuminuria/proteinuria at the baseline. Thus, we strongly recommend the RAS inhibitors as the first-line anti-hypertensive drugs for diabetic A2 or A3 category CKD.

In T2DM (type 2 diabetes mellitus) patients with normoalbuminuria (A1), ACE-I or ARB inhibited the development of micro-albuminuria, particularly in the presence of hypertension. However, there have been no large-scale studies investigating the relative renal or cardiovascular protective effects of RAS inhibitors and other classes of anti-hypertensive drugs with a head-to-head comparison in diabetic CKD patients with reduced GFR and normal urinary albumin excretion. Thus, we tentatively suggest the RAS inhibitors as first-line anti-hypertensive drugs for diabetic CKD with normo-albuminuria (A1).

To achieve the recommended clinic BP target, combination therapy should be considered. When mono-therapy with a RAS inhibitor is not sufficient to achieve the target $\mathrm{BP}$, combination therapy employing a RAS inhibitor together with a long-acting $\mathrm{CCB}$, a thiazide-type diuretic (thiazides and thiazide-like agents for G1, G2 or G3 category) or a loop diuretic (for G4 or G5 category) should be considered. In CKD G4 or G5, a combination of a thiazide diuretic and a loop diuretic may be considered to obtain adequate diuresis while exerting due caution for possible adverse effects, such as renal deterioration, hyponatremia and hypokalemia.

2. First-line anti-hypertensive drugs for non-diabetic CKD

In non-diabetic A1 category CKD, no convincing evidence exists to demonstrate the superior benefits of ARBs or ACE inhibitors over other classes of anti-hypertensive drugs. A meta-analysis of patient-level data also showed the beneficial effect of ACE-I in slowing the progression of nondiabetic CKD with higher baseline urinary protein excretion. Furthermore, ARB reduced the incidence of renal events compared with CCB therapy in Japanese high-risk hypertensive patients with $\mathrm{G} 4$ category CKD and proteinuria.

Therefore, for non-diabetic A1 category CKD, ARBs, ACE inhibitors, CCBs or diuretics are recommended as preferred anti-hypertensive drugs (Grade B). On the other hand, RAS inhibition has been shown to be particularly beneficial for renoprotection in non-diabetic CKD patients with proteinuria (A2 and $\mathrm{A} 3$ categories), and the presence of proteinuria in non-diabetic CKD patients is a rationale for priority of the RAS inhibitors as first-line anti-hypertensive drugs (Grade B).

\section{Bibliography}

1. Casas JP, et al. Lancet. 2005;366:2026-33. (Level 1)

2. Holtkamp FA. Eur Heart J. 2011;12:1493-9. (Level 2)

3. Ruggenenti P, et al. N Engl J Med. 2004;351:1941-51. (Level 2)

4. Haller H, et al. N Engl J Med. 2011;364:907-17. (Level 2)

5. Bakris GL, et al. Am J Kidney Dis. 2000;36:646-61. (Level 4)

6. Rahman M, et al. Clin J Am Soc Nephrol. 2012;7:989-1002. (Level 4)

7. Jafar TH, et al. Ann Intern Med. 2003;139:244-52. (Level 4)

8. Appel LJ, et al. N Engl J Med. 2010;363:918-29. (Level 4)

9. The GISEN Group (Gruppo Italiano di Studi Epidemiologici in Nefrologia). Lancet. 1997;349:1857-63. (Level 2)

10. Jafar TH, et al. Ann Intern Med. 2001;135:73-87. (Level 1)

11. Hou FF, et al. N Engl J Med. 2006;354:131-40. (Level 2)

12. Saruta T, et al. Hypertens Res. 2009;32:505-12. (Level 2)

13. Agodoa LY, et al. JAMA. 2001;285:2719-28. (Level 2)

14. Viberti G, et al. Circulation. 2002;106:672-8. (Level 2)

15. The EUCLID Study Group. Lancet. 1997;349:1787-92. (Level 2)

16. Parving HH, et al. N Engl J Med. 2001;345:870-8. (Level 2)

17. Lewis EJ, et al. N Engl J Med. 1993;329:1456-62. (Level 2)

18. Lewis EJ, et al. N Engl J Med. 2001;345:851-60. (Level 2)

19. Brenner BM, et al. N Engl J Med. 2001;345:861-9. (Level 2)

20. Mann JF, et al. Am J Kidney Dis. 2003;42:936-42. (Level 2)

21. Heart Outcomes Prevention Evaluation Study Investigators. Lancet. 2000;355:253-9. (Level 2)

22. Kunz R, et al. Ann Intern Med. 2008;148:30-48. (Level 1)

23. Imai E, et al. Diabetologia. 2011;54:2978-86. (Level 2)

24. MacKinnon M, et al. Am J Kidney Dis. 2006;48:8-20. (Level 1)

25. Tobe SW, et al. Circulation. 2011;123:1098-107. (Level 2)

26. Mann JF, et al. Lancet. 2008;372:547-53. (Level 2)

27. The ONTARGET Investigators. N Engl J Med. 2008;358:1547-59. (Level 2)

28. Wright JT Jr, et al. JAMA. 2002;288:2421-31. (Level 2)

29. Contreras G, et al. Hypertension. 2005;46:44-50. (Level 2)

30. Iino Y, et al. Hypertens Res. 2004;27:21-30. (Level 2)

31. Schjoedt KJ. Kidney Int. 2006;70:536-42. (Level 2)

32. White WB, et al. Hypertension. 2003;41:1021-6. (Level 2)

33. Navaneethan SD, et al. Clin J Am Soc Nephrol. 2009;4:542-51. (Level 1)

34. Mehdi UF, et al. J Am Soc Nephrol. 2009;20:2641-50. (Level 2)

35. Parving HH, et al. N Engl J Med. 2008;358:2433-46. (Level 2)

36. Parving HH, et al. N Engl J Med. 2012;367:2204-13. (Level 2)

37. Bakris GL, et al. Lancet. 2010;375:1173-81. (Level 2)

38. Jamerson K, et al. N Engl J Med. 2008;359:2417-28. (Level 2)

39. Webb AJ, et al. Lancet. 2010;375:906-15. (Level 1)

40. Fujita T, et al. Kidney Int. 2007;72:1543-9. (Level 2)

41. Pitt B, et al. Circulation. 2000;102:1503-10. (Level 2)

42. Julius S, et al. Lancet. 2004;363:2022-31. (Level 2)

43. Nissen SE, et al. JAMA. 2004;292:2217-25. (Level 2)

44. Packer M, et al. N Engl J Med. 1996;335:1107-14. (Level 2)

45. de Leeuw PW, et al. Arch Intern Med. 2004;164:2459-64. (Level 2)

46. Schrier RW, et al. Kidney Int. 2002;61:1086-97. (Level 2)

47. Hasebe N, et al. J Hypertens. 2005;23:445-53. (Level 2)

48. Abe M, et al. Hypertens Res. 2011;34:268-73. (Level 2)

49. Uzu T, et al. J Hypertens. 2005;23:861-5. (Level 4) 
50. 51. ALLHAT Officers and Coordinators for the ALLHAT Collaborative Research Group. JAMA. 2002;288:2981-97. (Level 2)

51. Law MR, et al. BMJ. 2003;326:1427-31. (Level 1)

52. Bakris GL, et al. Kidney Int. 2008;73:1303-9. (Level 2)

\section{Chapter 5: Nephrosclerosis}

CQ1 Is antihypertensive treatment recommended for nephrosclerosis?

We recommend appropriate antihypertensive treatment that reduces the progression of renal dysfunction for adults with nephrosclerosis. (Grade A) We recommend that adults with nephrosclerosis with proteinuria of $<0.15 \mathrm{~g} / \mathrm{gCr}$ (A1 category) be treated with blood pressure-reducing drugs to maintain a consistent blood pressure of $<140 / 90 \mathrm{mmHg}$. (Grade A)

We recommend that adults with nephrosclerosis with proteinuria of $\geq 0.15 \mathrm{~g} / \mathrm{gCr}$ (A2 and 3 categories) be treated with blood pressure-reducing drugs to maintain a consistent blood pressure of $<130 / 80 \mathrm{mmHg}$. (Grade C1)

The AASK study examined the effect of antihypertensive treatment on 1,094 enrolled African American patients with hypertensive nephrosclerosis. No such trial has yet been conducted to study Japanese patients. The study had a $3 \times 2$ factorial design with patients randomly assigned to low (mean arterial pressure (MAP) $<92 \mathrm{mmHg}$ ) or usual (MAP 102-107 mmHg) blood pressure targets, and administered any one of the three initial therapies, ACEIs, $\beta$-blockers, or CCBs. Since the AASK study suggested that lower blood pressure was associated with the prevention of progression of $\mathrm{CKD}$, we recommend antihypertensive treatment for adults with nephrosclerosis.

In a random period of the AASK trial, the average rate of change (as a slope) in GFR did not differ between the low and usual blood pressure groups (MAP $<92 \mathrm{mmHg}$ and 102-107 $\mathrm{mmHg}$, respectively) and the low and high proteinuria groups $(<0.22 \mathrm{~g} / \mathrm{gCr}$ and $>0.22 \mathrm{~g} / \mathrm{gCr}$, respectively). In the post-trial follow-up period of AASK, there was a difference between the low and usual blood pressure groups and in the progression of kidney disease in the group with proteinuria $(>0.22 \mathrm{~g} / \mathrm{gCr})$, but there was no difference in the group without proteinuria. Additionally, in the extended follow-up period of the AASK trial, low levels of proteinuria at baseline and randomization for the lower blood pressure goals were associated with an increase in eGFR.
From these findings, we recommend that adults with nephrosclerosis with proteinuria of $<0.15 \mathrm{~g} / \mathrm{gCr}$ (A1 category) be treated with BP-reducing drugs to maintain a consistent blood pressure of $<140 / 90 \mathrm{mmHg}$. Furthermore, we suggest that adults with nephrosclerosis with proteinuria of $0.15-0.5 \mathrm{~g} / \mathrm{gCr}$ (A2 category) and $\geq 0.5 \mathrm{~g} / \mathrm{gCr}$ (A3 category) be treated with blood pressure -reducing drugs to maintain a consistent blood pressure of $<130 / 80 \mathrm{mmHg}$.

\section{Bibliography}

1. Fogo A, et al. Kidney Int. 1997;51:244-52.

2. Agodoa LY, et al. JAMA. 2001;285:2719-28. (Level 2)

3. Wright JT Jr, et al. JAMA. 2002;288:2421-31. (Level 2)

4. Contreras G, et al. Hypertension. 2005;46:44-50. (Level 2)

5. Lea J, et al. Arch Intern Med. 2005;165:947-53. (Level 2)

6. Norris K, et al. Am J Kidney Dis. 2006;48:739-51. (Level 2)

7. Appel LJ, et al. Arch Intern Med. 2008;168:832-9. (Level 4)

8. Appel LJ, et al. N Engl J Med. 2010;363:918-29. (Level 4)

9. Upadhyay A, et al. Ann Intern Med. 2011;154:541-8. (Level 4)

10. Toto RD, et al. Kidney Int. 1995;48:851-9. (Level 2)

11. Hu B, et al. J Am Soc Nephrol. 2012;23:706-13. (Level 4)

CQ2 Which antihypertensive drugs are recommended as preferred medications for the management of hypertension in adults with nephrosclerosis?

We recommend that an ARB or an ACEI be used for hypertension in adults with nephrosclerosis with proteinuria of $0.15-0.5 \mathrm{~g} / \mathrm{gCr}$ (A2 category) or $\geq 0.5 \mathrm{~g} / \mathrm{gCr}$ (A3 category). (Grade B)

In adults with nephrosclerosis with proteinuria of $<0.15 \mathrm{~g} / \mathrm{gCr}$ (A1 category), we recommend ARBs, ACEIs, CCBs, and diuretics as the preferred antihypertensive drugs. (Grade B)

In the AASK trial, an ACEI was beneficial for patients with proteinuria compared with a $\mathrm{CCB}$ and retarded the progression of renal disease in patients with hypertensive renal disease and proteinuria. The findings of the AASK trial suggest that ARBs or ACEIs can be used in adults with nephrosclerosis with proteinuria of $0.15-0.5 \mathrm{~g} / \mathrm{gCr}$ (A2 category) or $\geq 0.5 \mathrm{~g} / \mathrm{gCr}$ ( $\mathrm{A} 3$ category) who are prescribed treatment with blood pressure-reducing drugs. The renoprotective benefit of ACEIs in these participants without proteinuria was less definitive compared with that of CCBs or $\beta$-blockers. In the 8-12-year post-trial follow-up period of the AASK trial, patients were treated to achieve a blood pressure of $<130 / 80 \mathrm{mmHg}$ with either ACEIs or ARBs if the patient was ACEI-intolerant. There was no difference between the groups in terms of the progression of CKD. Patients with higher levels of proteinuria $(>1 \mathrm{~g} / 24 \mathrm{~h})$ but not those with low levels of proteinuria, had a slower rate 
of kidney function loss when randomized to the more stringent blood pressure target control group. These findings are similar to the findings of the ALLHAT, LIFE, and TRANCEND trials, suggesting that ARBs or ACEIs can be used for adults with nephrosclerosis with proteinuria of $0.15-0.5 \mathrm{~g} / \mathrm{gCr}$ (A2 category) or $\geq 0.5 \mathrm{~g} / \mathrm{gCr}$ (A3 category); however, these groups of drugs are less effective for the A1 category $(<0.15 \mathrm{~g} / \mathrm{gCr})$. Therefore, we also recommend $\mathrm{CCB}$ and diuretics as the preferred blood pressure-reducing drugs for the A1 category.

\section{Bibliography}

1. Fogo A, et al. Kidney Int. 1997;51:244-52.

2. Agodoa LY, et al. JAMA. 2001;285:2719-28. (Level 2)

3. Wright JT Jr, et al. JAMA. 2002;288:2421-31. (Level 2)

4. Contreras G, et al. Hypertension. 2005;46:44-50. (Level 2)

5. Lea J, et al. Arch Intern Med. 2005;165:947-53. (Level 2)

6. Norris K, et al. Am J Kidney Dis. 2006;48:739-51. (Level 2)

7. Appel LJ, et al. Arch Intern Med. 2008;168:832-9. (Level 4)

8. Appel LJ, et al. N Engl J Med. 2010;363:918-29. (Level 4)

9. Upadhyay A, et al. Ann Intern Med. 2011;154:541-8. (Level 4)

10. Toto RD, et al. Kidney Int. 1995;48:851-9. (Level 2)

11. Hu B, et al. J Am Soc Nephrol. 2012;23:706-13. (Level 4)

\section{Chapter 6: Renal artery stenosis}

CQ1 Which methods are recommended

for the diagnosis of renal artery stenosis?

We recommend duplex ultrasonography, computed tomographic angiography or magnetic resonance angiography (MRA) as a screening test to diagnose renal artery stenosis.

When the results of noninvasive clinical tests are suggestive but inconclusive, we recommend catheter angiography as a diagnostic test for renal artery stenosis.

1. Summary ROC curves revealed that computed tomography angiography and gadolinium-enhanced, threedimensional magnetic resonance angiography are significantly better than duplex ultrasonography. However, duplex ultrasonography is an inexpensive and widely available test. The usefulness and reliability of Doppler ultrasound partly depends on the specific operator and the time allotted for optimal studies. Its main drawbacks relate to the difficulties of obtaining adequate data in obese patients and in patients with multi-vessel renal arteries.

2. Gadolinium-enhanced imaging of the abdominal and renal vasculature has been used as a tool for diagnosing renovascular diseases at many institutions. Concerns about potential adverse effects of gadolinium-based contrast for imaging, such as nephrogenic systemic fibrosis, have effectively eliminated contrast-enhanced magnetic resonance imaging for patients with eGFR $<30 \mathrm{ml} / \mathrm{min} / 1.73 \mathrm{~m}^{2}$. Current multi-detector computed tomography studies allow for excellent image resolution with rapid acquisition and less contrast exposure than before. Intra-arterial and intrarenal arterial angiography currently remain the gold standard for imaging vascular anatomy and stenotic lesions in the kidney at the time of a planned intervention, such as endovascular angioplasty and/or stenting.

\section{Bibliography}

1. Vasbinder GB, et al. Ann Intern Med. 2001;135:401-11. (Level 4)

2. Olin JW, et al. Ann Intern Med. 1995;122:833-8. (Level 4)

3. Williams GJ, et al. Am J Roentgenol. 2007;188:798-811. (Level 4)

4. Radermacher J, et al. N Engl J Med. 2001;344:410-7. (Level 4)

5. Zeller T, et al. Catheter Cardiovasc Interv. 2003;58:510-5. (Level 4)

6. Ikee R, et al. Am J Kidney Dis. 2005;46:603-9. (Level 4)

7. Ng YY, et al. J Chin Med Assoc. 2010;73:300-7. (Level 4)

8. Khoo MM, et al. Eur Radiol. 2011;21:1470-6. (Level 4)

9. Vasbinder GB, et al. Ann Intern Med. 2004;141:674-82. (Level 3)

CQ2 Is antihypertensive therapy recommended for managing renal artery stenosis in patients with CKD?

We strongly recommend antihypertensive therapy for the management of renal artery stenosis with CKD to slow the progression of renal dysfunction. (Grade A)

RAS inhibitors, calcium channel blockers and diuretics are recommended as the preferred antihypertensive drugs in patients with unilateral renal artery stenosis; however, information from prospective, randomized trials that have compared these medications is limited for renovascular disease. From cohort data, improved survival and decreased CVD events were found to be associated with the use of ACEIs in revascularized and medically-treated patients. Use of RAS inhibitors is contraindicated in patients with bilateral renal artery stenosis because of possible subsequent renal deterioration. When hyperkalemia, hypotension or symptoms/signs of hypoperfusion of organs emerges with use of RAS inhibitors, dose reduction or discontinuation of the drugs should be considered. 


\section{Bibliography}

1. Kalra PA, et al. Kidney Int. 2010;77:37-43. (Level 4)

2. Hackam DG, et al. Am Heart J. 2008;156:549-55. (Level 4)

3. Losito A, et al. Nephrol Dial Transplant. 2005;20:1604-9. (Level 4)

4. van de Ven PJ, et al. Kidney Int. 1998;53:986-93. (Level 4)

5. Cooper CJ, et al. Am Heart J. 2006;152:59-66. (Level 2)

CQ3 Is percutaneous revascularization combined with medical therapy recommended for the treatment of patients with renal artery stenosis and CKD?

We tentatively suggest percutaneous revascularization combined with medication for the treatment of patients with hemodynamically significant renal artery stenosis and CKD. (Grade C1)

1. After a comparison of BP changes occurring after renal revascularization reported by several RCTs and metaanalyses, renal revascularization was found to be effective for reducing BP and improving renal function and the patients' prognoses. The usefulness of percutaneous revascularization for renal artery stenosis is not yet wellestablished and has not been proved to be more effective than antihypertensive medication alone. However, there have been beneficial effects of revascularization in selected patients, particularly in those with bilateral kidney disease. We advise that adverse effects of revascularization be considered carefully.

2. Two RCTs (STAR and ASTRAL trials) showed no evidence of any significant clinical benefit of revascularization in the BP control, renal prognosis or CVD events, compared to medication.

3. The results of clinical trials indicate that the benefits of endovascular procedures are moderate compared with effective antihypertensive medication. Patients failing to respond to medication often show improved BP control after revascularization for heart failure. From these findings, we suggest that percutaneous revascularization be used to treat patients with hemodynamically significant renal artery stenosis.

\section{Bibliography}

1. Plouin PF, et al. Hypertension 1998; 31: 823-9. (Level 2) EMMA trial

2. Webster J, et al. J Hum Hypertens. 1998;12:329-35. (Level 2) SNRASCG trial

3. van Jaarsveld BC, et al. N Engl J Med. 2000;342:1007-14. (Level 2) DRASTIC trial
4. Ives NJ, et al. Nephrol Dial Transplant. 2003;18:298-304. (Level 1)

5. Losito A, et al. Nephrol Dial Transplant. 2005;20:1604-9. (Level 4)

6. Balk E, et al. Ann Intern Med. 2006;145:901-12. (Level 4)

7. Bax L, et al. Ann Intern Med. 2009;150:840-8. (Level 2) STAR trial

8. The ASTRAL Investigators. N Engl J Med. 2009;361:1953-62. (Level 2) ASTRAL trial

9. Ronden RA, et al. J Hypertens. 2010;28:2370-7. (Level 1)

10. Cooper CJ, et al. Am Heart J. 2006;152:59-66. (Level 2) CORAL trial

\section{Chapter 7: Renal anemia}

CQ1 Is treatment with Erythropoiesis-Stimulating Agent (ESA) recommended for renal anemia in nondialysis CKD?

We recommend ESA treatment for renal anemia in non-dialysis CKD patients, because it can be expected to improve the QOL. (Grade B)

It is not clear whether ESA treatment can prevent the progression of CKD and decrease the incidence of CVD in non-dialysis CKD.

We do not recommend $\mathrm{Hb}$ level $>13.0 \mathrm{~g} / \mathrm{dL}$ in ESA treatment, because this condition may increase the incidence of CVD in non-dialysis CKD. (Grade D)

ESA treatment is reasonable for renal anemia because a major cause of renal anemia is a deficiency of erythropoietin. Despite the unclear effects of ESA treatment on the progression of CKD and the incidence of CVD, many studies have demonstrated that ESA treatment for renal anemia in CKD improves the QOL. Therefore, we recommend ESA treatment for renal anemia in CKD. However, because some recent large RCTs, such as TREAT, CREATE and CHOIR, showed that CVD events increased in the group with a higher $\mathrm{Hb}$ target $(>13 \mathrm{~g} /$ $\mathrm{dL})$ as compared to the group with a lower $\mathrm{Hb}$ target $(9-11 \mathrm{~g} / \mathrm{dL})$, ESA treatment with a target $\mathrm{Hb}$ level exceeding $13.0 \mathrm{~g} / \mathrm{dL}$ is not recommended for renal anemia in CKD patients.

\section{Bibliography}

1. Pfeffer MA, et al. N Engl J Med. 2009;361:2019-32. (Level 2)

2. Drüeke TB, et al. N Engl J Med. 2006;355:2071-84. (Level 2)

3. Singh AK,et al. N Engl J Med. 2006;355:2085-98. (Level 2)

4. Akizawa T, et al. Ther Apher Dial. 2011;15:431-40. (Level 2) 
CQ2 Is ESA treatment for renal anemia effective for preventing CKD progression and decreasing the incidence of CVD?

Some reports have suggested that ESA treatment for renal anemia was effective for preventing CKD progression and decreasing the incidence of CVD. ESA treatment targeting Hb levels $>12-13 \mathrm{~g} / \mathrm{dL}$ did not seem to be effective for preventing CKD progression or decreasing the incidence of CVD compared to the Hb level of 9-11.5 g/dL, but rather had the potential to lead to an increase in the incidence of CVD.

Recent large RCTs conducted overseas demonstrated that groups with higher $\mathrm{Hb}$ levels did not show effectiveness in terms of preventing the progression of CKD and decreasing the incidence of CVD compared to groups with lower $\mathrm{Hb}$ levels. A meta-analysis including these RCTs concluded that targeting higher $\mathrm{Hb}$ levels $(>12-13 \mathrm{~g} / \mathrm{dL}$ ) probably increases the risk of death, serious cardiovascular events and end-stage renal disease. In contrast, a Japanese RCT demonstrated that groups with a higher $\mathrm{Hb}$ level (11-13 g/dL) treated with darbepoetin had a more favorable outcome in terms of preventing the progression of CKD and cardiac hypertrophy compared to groups with a lower $\mathrm{Hb}$ (9-11 g/dL) treated by rHuEPO. Further analysis is necessary to clarify this issue.

\section{Bibliography}

1. Kuriyama S, et al. Nephron. 1997;77:176-85. (Level 2)

2. Tsubakihara Y, et al. Ther Apher Dial. 2012;16:529-40. (Level 2)

3. Gouva C,et al. Kidney Int. 2004;66:753-60. (Level 2)

4. Cody J,et al. Cochrane Database Syst Rev. 2005;3:CD003266. (Level 1)

5. Palmer SC, et al. Ann Intern Med. 2010;153:23-33. (Level 1)

6. Drüeke TB, et al. N Engl J Med. 2006;355:2071-84. (Level 2)

7. Singh AK,et al. N Engl J Med. 2006;355:2085-98. (Level 2)

8. Pfeffer MA, et al. N Engl J Med. 2009;361:2019-32. (Level 2)

9. Akizawa T, et al. Ther Apher Dial. 2011;15:431-40. (Level 2)

10. Roger SD, et al. J Am Soc Nephrol. 2004;15:148-56. (Level 2)

11. Levin A,et al. Am J Kidney Dis. 2005;46:799-811. (Level 2)

12. Rossert J, et al. Am J Kidney Dis. 2006;47:738-50. (Level 2)

13. Macdougall IC, et al. Nephrol Dial Transplant. 2007;22:784-93. (Level 2)

14. Ritz E, et al. Am J Kidney Dis. 2007;49:194-207. (Level 2)

15. Cianciaruso B, et al. J Nephrol. 2008;21:861-70. (Level 2)

16. Besarab A, et al. N Engl J Med. 1998; 339:584-90. (Level 2)

17. Ngo K, et al. Cochrane Database Syst Rev. 2010;1:CD007613. (Level 1)
CQ3 Are higher doses of ESA recommended for renal anemia in non-dialysis CKD?

We do not recommend higher doses of ESA for renal anemia in non-dialysis CKD, because the risk of CVD might increase. (Grade D)

From large clinical trials on ESA treatment in nondialysis CKD patients, it has been reported that a higher $\mathrm{Hb}$ target increased the risk of CVD events. From this result, there were concerns that higher doses of ESA might cause higher incidence of CVD events.

There is no clear definition of what constitutes a high dose of ESA in the treatment of renal anemia at present. However, the above-mentioned results suggested that higher doses of ESA might have led to the higher incidence of CVD events in non-dialysis CKD. Until now, it has not been clear whether a higher $\mathrm{Hb}$ target or a higher dose of ESA presents a risk for CVD events. In addition, low responsiveness to ESA is probably a factor involved in this problem. In general, patients with low responsiveness to ESA require higher doses of ESA, thus low responsiveness to ESA is also a possible cause of a higher incidence of CVD events. We cannot determine whether or not the higher doses of ESA are the cause of a higher incidence of CVD events, hence the use of higher doses of ESA should be avoided at this time.

\section{Bibliography}

1. Drüeke TB, et al. N Engl J Med. 2006;355:2071-84. (Level 2)

2. $\quad$ Singh AK,et al. N Engl J Med. 2006;355:2085-98. (Level 2)

3. Pfeffer MA, et al. N Engl J Med. 2009;361:2019-32. (Level 2)

4. Palmer SC, et al. Intern Med. 2010;153:23-33. (Level 1)

5. Villar E, et al. J Diabetes Complicat. 2011;25:237-43. (Level 2)

6. Akizawa T, et al. Ther Apher Dial. 2011;15:431-40. (Level 2)

7. Szczech LA, et al. Kidney Int. 2008;74:791-8. (Level 2)

8. Solomon SD, et al. N Engl J Med. 2010;363:1146-55. (Level 2)

9. Skali H, et al. Circulation. 2011;124:2903-8. (Level 2)

CQ4 Is iron treatment recommended for renal anemia?

We suggest that iron supplementation be considered as a treatment for renal anemia in patients with suspected iron deficiency. However, the safety margin for iron supplementation is unclear. (Grade C1)

It is important to diagnose and correct iron deficiency because iron treatment has the potential to yield a meaningful erythropoietic response in CKD patients. On the other hand, iron supplementation carries the risk of several disorders if there is an iron overdose. Serum ferritin and TSAT (Fe/TIBC) are widely used to estimate body iron 
stores in spite of their limited diagnostic power. There is only limited evidence in patients with CKD that serves as a guide for defining a specific upper limit of the target range for iron treatment. Therefore, at present, it is difficult to assess iron status precisely and avoid an iron overdose. Consequently the guidelines of several countries have each proposed criteria for iron treatment. The decision to administer iron to an individual patient should be based on the assessment that the potential adverse effects of iron supplementation are appropriately outweighed by the expected benefits of treatment.

\section{Bibliography}

1. Kovesdy CP, et al. Clin J Am Soc Nephrol. 2009;4:435-41. (Level 4)

2. Kalantar-Zadeh K, et al. J Am Soc Nephrol. 2005;16:3070-80. (Level 4)

3. Pollak VE, et al. BMC Nephrol. 2009;10:6. (Level 4)

4. Teehan GS, et al. Clin Infect Dis. 2004:38:1090-4. (Level 4)

5. Hasuike Y, et al. Clin Exp Nephrol. 2010;14:349-55. (Level 4)

6. Stancu S, et al. Am J Kidney Dis. 2010;55:639-47. (Level 4)

CQ5 Are long-acting ESAs recommended for treatment of renal anemia in non-dialysis CKD?

We suggest that treatment using long-acting ESAs can be considered for renal anemia in non-dialysis CKD patients. (Grade C1)

Recently, long-acting ESAs have become available. The advantage of these new ESAs was examined.

Since long-acting ESAs have a longer half-life as compared to recombinant human erythropoietin (rHuEPO), improving and maintaining the $\mathrm{Hb}$ level through a lower frequency of administration can be expected. At the same time, long-acting ESA might change the clinical outcome as a result of the different function and duration of activity. However, the latter is not clear at present. For the former statement, a cohort study on darbepoetin alfa (DA) by Gobin et al. has been the only one to report that the frequency of administration necessary for achieving the target $\mathrm{Hb}$ was decreased by replacing rHuEPO with long-acting ESA in non-dialysis CKD. A randomized controlled trial comparing DA with rHuEPO has not been conducted, so the absolute superiority of DA over rHuEPO has not been demonstrated. The status of methoxy polyethylene glycolepoetin beta is also the same. Although a randomized controlled trial has been conducted, it merely confirmed that administration every 4 weeks did not yield inferior results compared with administration every 2 weeks. As mentioned above, we conclude that currently there is no strong reason to recommend long-acting ESAs.

\section{Bibliography}

1. Gobin J, et al. Clin Drug Investig. 2011;31:113-20. (Level 4)

2. Hertel J, et al. Am J Nephrol. 2006;355-26:149-56. (Level 4)

3. Disney A, et al. Nephrology. 2007;12:95-101. (Level 4)

4. Agarwal AK, et al. J Intern Med. 2006;260:577-85. (Level 4)

5. Kessler M, et al. Hemodial Int. 2010;14:233-9. (Level 2)

6. Roger SD, et al. Nephrol Dial Transplant. 2011;26:3980-6. (Level 2)

\section{Chapter 8: CKD-Mineral and Bone Disorders (MBD)}

CQ1 Is targeting serum phosphate within the normal range recommended for CKD patients?

We recommend that serum phosphate levels should be targeted within the reference range irrespective of the CKD stage, because a higher serum phosphate level is associated with poorer overall and renal outcomes. However, precise target levels and desirable methods of intervention have not yet been established. (Grade C1)

One recent meta-analysis showed that a $1 \mathrm{mg} / \mathrm{dL}$ increase in the serum phosphate level was associated with a $29 \%$ increase in all-cause mortality in CKD patients. A sub-analysis using a limited number of well-designed studies with multiple covariates demonstrated an even higher hazardss ratio of 1.35. Due to a lack of evidence, the association of serum phosphate with cardiovascular death in CKD patients remains to be elucidated.

In other reports, a high serum phosphate level was associated with a steeper decline in eGFR and an increased risk of ESRD in CKD patients.

Furthermore, since a higher serum phosphate level even within the reference range is associated with cardiovascular events and development and progression of CKD in the general population, it would seem prudent to state that a high serum phosphate should be avoided.

However, there have been no reported RCTs that directly compared the overall and renal outcomes prospectively in different phosphate-level arms. Therefore, there is no evidence about the extent to which the phosphate level should be lowered.

Recently, FGF23, a newly-found phosphaturic hormone, has been demonstrated to be a strong prognostic marker of overall, cardiovascular, and renal outcomes in CKD patients. An increase in the level of FGF23 in the serum is known to precede that of phosphate and is evoked by daily oral phosphorus intake. Accordingly, even within the reference range of phosphate, some CKD patients could be at risk of a phosphate overload and subsequently a poorer outcome. Thus, theoretically it is preferable to keep the 
level of serum phosphate as low as possible within the reference range in CKD patients.

Since there is very little evidence demonstrating the benefit of treatment or modification of diet to achieve lower serum phosphate levels in CKD patients, no recommendation for specific intervention is provided here. More studies are required.

\section{Bibliography}

1. Block GA, et al. J Am Soc Nephrol. 2004;15:2208-18. (Level 4)

2. Young EW, et al. Kidney Int. 2005;67:1179-87. (Level 4)

3. Kalantar-Zadeh K, et al. Kidney Int. 2006;70:771-80. (Level 4)

4. Floege J, et al. Nephrol Dial Transplant. 2011;26:1948-55. (Level 4)

5. Palmer SC, et al. JAMA. 2011;305:1119-27. (Level 4)

6. Schwarz S, et al. Clin J Am Soc Nephrol. 2006;1:825-31. (Level 4)

7. Tangri N, et al. JAMA. 2011;305:1553-9. (Level 4)

8. Voormolen N, et al. Nephrol Dial Transplant. 2007;22:2909-16. (Level 4)

9. Chue CD, et al. Nephrol Dial Transplant. 2011;26:2576-82. (Level 4)

10. Moore J, et al. Clin Transplant. 2011;25:406-16. (Level 4)

11. Sampaio MS, et al. Clin J Am Soc Nephrol. 2011;6:2712-21. (Level 4)

12. Dhingra R, et al. Arch Intern Med. 2007;167:879-85. (Level 4)

13. O'Seaghdha CM, et al. Nephrol Dial Transplant. 2011;26:2885-90. (Level 4)

14. Isakova T, et al. Kidney Int. 2011;79:1370-8. (Level 4)

15. Nakano C, et al. Clin J Am Soc Nephrol. 2012;7:810-9. (Level 4)

16. Fliser D, et al. J Am Soc Nephrol. 2007;18:2600-8. (Level 4)

17. Parker BD, et al. Ann Intern Med. 2010;152:640-8. (Level 4)

18. Isakova T, et al. JAMA. 2011;305:2432-9. (Level 4)

19. Wolf M, et al. J Am Soc Nephrol. 2011;22:956-66. (Level 4)

20. Murtaugh MA, et al. Nephrol Dial Transplant. 2012;27:990-6. (Level 4)

21. Kovesdy CP, et al. Am J Kidney Dis. 2010;56:842-51. (Level 4)

CQ2 Do serum parathyroid hormone (PTH) levels affect the mortality of patients with CKD?

Whether or not PTH levels affect the mortality of patients with CKD remains unclear.

Many studies have demonstrated that phosphate is closely associated with all-cause and CVD mortality. However, the relationship between serum PTH levels and mortality in patients with CKD remains ambiguous. Only two papers of studies that investigated the relationship between serum PTH levels and mortality in patients with CKD have been found in a literature search. These studies were retrospective and included only a small number of patients with CKD. The results showed a significant relationship between serum PTH levels and mortality risk. However, in addition to a small number of study patients, the observational period was relatively short. The number of deaths was very large during such a short observational period, and these results are not thought to be applicable to Japanese patients with CKD. Furthermore, a meta-analysis including dialysis patients demonstrated that serum PTH was not significantly associated with mortality. Taken together, these mixed findings indicate that at present, the effect of serum PTH levels on the mortality of patients with CKD remains unclear.

\section{Bibliography}

1. Palmer SC, et al. JAMA. 2011;305:1119-27. Review. (Level 4)

2. Kovesdy CP, et al. Kidney Int. 2008;73:1296-302. (Level 4)

3. Smith DH, et al. J Bone Miner Metab. 2009;27:287-94. (Level 4)

CQ3 Is vascular calcification associated with an increased risk of CVD in patients with CKD?

Vascular calcification appears to be associated with an increased risk of CVD in patients with CKD.

Vascular calcification is an important finding that is related to various clinical problems. It is well known that vascular calcification is a crucial risk factor for CVD and mortality in dialysis patients. However, detailed data in non-dialysis patients with CKD are lacking. Only two papers in a literature search have shown a relationship between vascular calcification and CVD. Though these two studies included only a small number of study patients and were observational and prospective, their results demonstrated that coronary artery calcification was significantly correlated with CVD and mortality. In addition, a metaanalysis and large-scale studies including patients with and without CKD revealed that vascular calcification is significantly associated with increased all-cause and CVD mortality. Taken together, it is considered that vascular calcification is associated with an increased risk of CVD even in non-dialysis patients with CKD.

\section{Bibliography}

1. Rennenberg RJ, et al. Vasc Health Risk Manag. 2009;5:185-97. (Level 4)

2. Watanabe R, et al. Clin J Am Soc Nephrol. 2010;5:189-94. (Level 4)

3. Chiu YW, et al. Kidney Int. 2010;77:1107-14. (Level 4) 
CQ4 Is taking vitamin D good for the kidney?

It is unclear whether or not an active form of vitamin $\mathrm{D}$ and/or its analogs can ameliorate the progression of CKD.

Vitamin D plays a crucial role in the progression of CKD and the development of hyperparathyroidism.

Several observational studies have reported that poor vitamin D status, which is diagnosed from a low serum hydroxyvitamin D level, is associated with an increased risk of all-cause mortality in CKD patients irrespective of their dialysis status and even in the general population. One meta-analysis clearly showed that the administration of cholecalciferol (not for prescription in Japan), a native form of vitamin D, improves overall survival in the general population, especially in elderly women. However, there was no subgroup analysis of CKD patients who were not yet on dialysis. Accordingly, it cannot be conclusively stated that taking cholecalciferol is beneficial for CKD patients.

According to the results of several observational studies, the administration of calcitriol or an active form of vitamin $\mathrm{D}$, which had long been conducted for controlling secondary hyperparathyroidism, was associated with lower all-cause and cardiovascular mortality in CKD patients independently of serum phosphate, calcium, and PTH levels. However, no RCT has yet been conducted to test the finding.

On the other hand, paricalcitol (not approved in Japan), a vitamin $\mathrm{D}$ analog that is less likely to cause hypercalcemia than calcitriol, demonstrated promising results in protecting cardiomyocytes in both experimental animal studies and human observational studies. Although a related RCT recently failed to achieve a clinically meaningful outcome in terms of cardiac remodeling, paricalcitol and other vitamin D analogs are still assumed to have a renoprotective effect by reducing the amount of proteinuria. Nevertheless, this assumption needs to be elucidated in future study.

Due to a lack of evidence from RCTs, administration of an active form of vitamin $\mathrm{D}$ or its analogs remains controversial in that it could ameliorate overall and renal outcomes, and could help control secondary hyperparathyroidism in CKD patients; however, it is important to note that the administration of $>0.5 \mu \mathrm{g} /$ day of alfacalcidol or $>0.25 \mu \mathrm{g} /$ day of calcitriol may induce an adverse event of hypercalcemia and subsequent kidney damage.

\section{Bibliography}

1. Levin A, et al. Kidney Int. 2007;71:31-8. (Level 4)

2. Nakano C, et al. Clin J Am Soc Nephrol. 2012;7:810-9. (Level 4)

3. Wolf M, et al. Kidney Int. 2007;72:1004-13. (Level 4)

4. Pilz S, et al. Am J Kidney Dis. 2011;58:374-82. (Level 4)
5. Melamed ML, et al. Arch Intern Med. 2008;168:1629-37. (Level 4)

6. Chonchol M, et al. Kidney Int. 2007;71:134-9. (Level 4)

7. Dobnig H, et al. Arch Intern Med. 2008;168:1340-9. (Level 4)

8. Bjelakovic G, et al. Cochrane Database Syst Rev. 2011:CD007470. (Level 1)

9. Shoji T, et al. Nephrol Dial Transplant. 2004;19:179-84. (Level 4)

10. Teng M, et al. J Am Soc Nephrol. 2005;16:1115-25. (Level 4)

11. Kalantar-Zadeh K, et al. Kidney Int. 2006;70:771-80. (Level 4)

12. Tentori F, et al. Kidney Int. 2006;70:1858-65. (Level 4)

13. Naves-Diaz M, et al. Kidney Int. 2008;74:1070-8. (Level 4)

14. Kovesdy CP, et al. Arch Intern Med. 2008;168:397-403. (Level 4)

15. Shoben AB, et al. J Am Soc Nephrol. 2008;19:1613-9. (Level 4)

16. Sugiura S, et al. Clin Exp Nephrol. 2010;14:43-50. (Level 4)

17. Thadhani R, et al. JAMA. 2012;307:674-84. (Level 2)

18. Agarwal R, et al. Kidney Int. 2005;68:2823-8. (Level 2)

19. Fishbane S, et al. Am J Kidney Dis. 2009;54:647-52. (Level 2)

20. 20. de Zeeuw D, et al. Lancet. 2010;376:1543-51. (Level 2)

\section{Chapter 9: Diabetic nephropathy}

CQ1 Are albuminuria and results of eGFR measurements early detection markers for diabetic nephropathy?

Measurement of albuminuria is essential for the early detection of diabetic nephropathy.

Measurement of eGFR is not useful for the early detection of diabetic nephropathy.

Early detection markers of diabetic nephropathy enable better prediction of the prognosis of CKD or CVD. Albuminuria is a good predictive marker for the progression of $\mathrm{CKD}$ and cardio-vascular events in diabetic patients. However, mild reduction of eGFR does not predict the progression of CKD and cardio-vascular events in diabetic patients.

Although albuminuria is a clinically good predictive marker for the prognosis of CKD or CVD, pathological changes of typical diabetic nephropathy are occasionally detected in patients with normoalbuminuria. Although $30 \mathrm{mg} / \mathrm{gCr}$ is now the upper limit of normoalbuminuria, this level should be re-estimated with new evidence in future. Furthermore, albuminuria is not specific for diabetic nephropathy. More sensitive and specific markers are necessary to detect early diabetic nephropathy.

\section{Bibliography}

1. Katayama S, et al. Diabetologia. 2011;54:1025-31. (Level 4)

2. Adler AI, et al. Kidney Int. 2003;63:225-32. (Level 4)

3. Agardh CD, et al. Diabetes Res Clin Pract. 1997;35:113-21. (Level 4)

4. Mogensen CE, et al. N Engl J Med. 1984;311:89-93. (Level 4)

5. Bruno G, et al.Diabetologia. 2007;50:941-8. (Level 4) 
6. Ninomiya T, et al. J Am Soc Nephrol. 2009;20:1813-21. (Level 4)

7. Bouchi R, et al. Hypertens Res. 2009;32:381-6. (Level 4)

8. MacIsaac RJ, et al. Diabetes Care. 2004;27:195-200. (Level 4)

9. Middleton RJ, et al. Nephrol Dial Transplant. 2006;21:88-92. (Level 4)

10. Hanai K, et al. Nephrol Dial Transplant. 2009;24:1884-8. (Level 4)

11. Caramori ML, et al. Diabetes. 2003;52:1036-40. (Level 4)

CQ2 Is tight glycemic control recommended for preventing the onset and progression of diabetic nephropathy?

We recommend tight glycemic control for preventing the onset and progression of early diabetic nephropathy. (Grade B)

The target for HbA1c is $<7.0 \%$ in early diabetic nephropathy. (Grade B)

In the advanced stage with overt nephropathy, the effect of tight glycemic control on the prevention of progression of diabetic nephropathy is not clear.

Chronic hyperglycemia is the main causal factor of diabetic vascular complications, including nephropathy. Previous landmark clinical studies (the DCCT and EDIC studies for type 1 diabetes, UKPDS, Kumamoto, ADVANCE, ACCORD and the VADT study for type 2 diabetes) showed that tight glycemic control prevents the onset and progression of early nephropathy, and the target for HbA1c is $<7.0 \%$.

There are no reports of prospective studies that examined the effect of blood glucose control at the advanced stage with overt nephropathy; therefore, the effect of tight glycemic control on the suppression of diabetic nephropathy is not clear.

\section{Bibliography}

1. The Diabetes Control and Complications Trial Research Group. N Engl J Med. 1993;329:977-86. (Level 2)

2. Ohkubo Y, et al. Diabetes Res Clin Pract. 1995;28:103-17. (Level 2)

3. UK Prospective Diabetes Study (UKPDS) Group. Lancet. 1998;352:837-53. (Level 2)

4. Ismail-Beigi $\mathrm{F}$, et al. Lancet. 2010;376:419-30. (Level 2)

5. Patel A, et al. N Engl J Med. 2008;358:2560-72. (Level 2)

6. Duckworth W, et al. N Engl J Med. 2009;360:129-39. (Level 2)

7. Writing Team for the Diabetes Control and Complications Trial/Epidemiology of Diabetes Interventions and Complications (EDIC) study. JAMA. 2003;290:2159-67. (Level 4)

8. Holman RR, et al. N Engl J Med. 2008;359:1577-89. (Level 4)

9. Boussageon R, et al. BMJ. 2011;343:d4169. (Level 1)

10. Hemmingsen B, et al. BMJ. 2011;343:d6898. (Level 1)

11. de Boer IH, et al. N Engl J Med. 2011;365:2366-76. (Level 4)
CQ3 Is tight glycemic control recommended for suppressing the onset of CVD in patients with diabetic nephropathy?

We recommend glycemic control for suppressing the onset of CVD in patients with diabetic nephropathy. (Grade $\mathrm{C} 1$ )

Glycemic control without hypoglycemia is important, and the target of glycemic control should be considered in relation to the risk for each individual patient.

Renal dysfunction, such as microalbuminuria and proteinuria, is recognized to be an independent risk factor for the onset of CVD. Patients with CKD, including diabetic nephropathy, often develop CVD.

The effect of glycemic control alone on the onset of CVD in patients with diabetic nephropathy is unclear. However, glycemic control might contribute to suppressing the onset of CVD as a core treatment in multifactorial intensive therapy for diabetic nephropathy, and is an important factor for achieving the remission of albuminuria. It should also be noted that tight glycemic control might increase serious hypoglycemia, and reportedly could be a risk factor for increased mortality and the development of CVD in type 2 diabetes. Therefore, glycemic control that avoids hypoglycemia is crucial, and the glycemic control target should be considered along with the risks to the individual patient.

\section{Bibliography}

1. Gaede P, et al. N Engl J Med. 2003;348:383-93. (Level 2)

2. Araki S, et al. Diabetes. 2005;54:2983-7. (Level 4)

3. Araki S, et al. Diabetes. 2007;56:1727-30. (Level 4)

4. Gaede P, et al. Nephrol Dial Transplant. 2004;19:2784-8. (Level 4)

CQ4 Which anti-diabetic medications are recommended as the first-line treatment for diabetic nephropathy?

There is no significant difference among anti-diabetic medications for suppressing the onset and progression of diabetic nephropathy. At the early stage of nephropathy, anti-diabetic medications need to be selected in consideration of the individual patient's diabetic pathophysiology. At the advanced stage of overt nephropathy with reduction of renal function, anti-diabetic medications should be selected in consideration of the individual patient's renal function.

In CKD complicated with diabetes, anti-diabetic medications should be selected inconsideration of the individual patient's renal function. 
Anti-diabetic medicines include insulin and GLP-1 receptor agonist as injectable agents, and sulfonylurea, glinide, thiazolidinedione, biguanide, $\alpha$-glucosidase inhibitior and dipeptidyl peptidase- 4 inhibitor as oral anti-diabetic agents. There is no significant difference among antidiabetic medications in terms of the onset and progression of diabetic nephropathy, so far. Therefore, it is necessary to select anti-diabetic agents to control glucose levels tightly taking into consideration the individual patient's diabetic pathophysiology at the early stage of nephropathy. So far, there has been no study conducted to compare directly the effects of anti-diabetic medications in terms of their suppression of the onset and progression of diabetic nephropathy.

At the advanced stage of overt nephropathy with a reduction in renal function, the risk of hypoglycemia might be increased. Therefore, a therapeutic agent for diabetes should be selected with consideration of the patient's renal function to avoid the occurrence of hypoglycemia.

\section{Bibliography}

1. UK Prospective Diabetes Study (UKPDS) Group. Lancet. 1998;352:837-53. (Level 2)

2. UK Prospective Diabetes Study (UKPDS) Group. Lancet. 1998;352:854-65. (Level 4)

3. Gerstein HC, et al. N Engl J Med. 2008;358:2545-59. (Level 2)

4. Patel A, et al. N Engl J Med. 2008;358:2560-72. (Level 2)

CQ5 Is salt restriction recommended for hypertensive diabetic patients with CKD?

To reduce blood pressure, we recommend reducing the salt intake of hypertensive diabetic patients with CKD. (Grade B)

We recommend salt restriction to below $6 \mathrm{~g} /$ day in hypertensive diabetic patients with CKD. (Grade B)

We suggest avoiding salt restriction below $3 \mathrm{~g} /$ day in hypertensive diabetic patients with CKD. (Grade C2)

It is well known that a dietary salt intake is positively associated with blood pressure in general hypertensive patients. In addition, the salt sensitivity of blood pressure increases in the majority of patients with CKD.

There is some evidence that a low salt diet reduces blood pressure and urinary albumin (protein) excretion in diabetic patients with CKD. In addition, a low salt diet is critical to optimize the efficacy of medication used to reduce blood pressure and urinary albumin (protein) excretion. Therefore, we recommend a low salt diet for hypertensive diabetic patients with CKD.
Volume depletion associated with intensive salt restriction should be avoided in hypertensive diabetic patients with CKD, especially in the elderly.

There is no conclusive evidence demonstrating that salt restriction reduces mortality and cardiovascular events in diabetic patients with CKD. Further studies are needed to address this issue.

\section{Bibliography}

1. Suckling RJ, et al. Cochrane Database Syst Rev. 2010:CD006763. (Level 1)

2. Mühlhauser I, et al. Diabetologia. 1996;39:212-9. (Level 2)

3. Dodson PM, et al. BMJ. 1989;298:227-30. (Level 2)

4. Strojek K, et al. Nephrol Dial Transplant. 2005;20:2113-9. (Level 2)

5. Imanishi M, et al. Diabetes Care. 2001;24:111-6. (Level 2)

6. Thomas MC, et al. Diabetes Care. 2011;34:861-6. (Level 4)

7. Ekinci EI, et al. Diabetes Care. 2011;34:703-9. (Level 4)

8. Houlihan CA, et al. Diabetes Care. 2002;25:663-71. (Level 2)

9. Bakris GL, et al. Ann Intern Med. 1996;125:201-4. (Level 2)

CQ6 Are RAS inhibitors recommended as the first-line drug for hypertensive diabetic patients with CKD?

We recommend RAS inhibitors as the first-line drug for hypertension to prevent the progression of CKD with diabetes. (Grade A)

Blood pressure control reduced the risk of cardiovascular events in patients with diabetic nephropathy. Renoprotective effects of RAS inhibitors beyond blood pressure control have been reported.

It has been reported that in diabetic patients with normoalbuminuria or microalbuminuria, RAS inhibitors prevented increase in the levels of albuminuria or proteinuria. In diabetic patients with macroalbuminuria, renal function was reported to be preserved by the administration of RAS inhibitors. In comparison with CCBs, RAS inhibitors showed similar or more reno-protective effects in diabetic patients with CKD. These data indicated that RAS inhibitors should be the first-line drug for hypertensive diabetic patients with CKD.

\section{Bibliography}

1. Turnbull F, et al. Lancet. 2003;362:1527-35. (Level 1)

2. Turnbull F, et al. J Hypertens. 2007;25:951-8. (Level 1)

3. Haller H, et al. N Engl J Med. 2011;364:907-17. (Level 2)

4. The BErgamo NEphrologic DIabetes Complications Trial (BENEDICT) Control Clin Trials. 2003;24:442-61. (Level 2)

5. The EUCLID Study Group. Lancet. 1997;349:1787-92. (Level 2)

6. Sano T, et al. Diabetes Care. 1994;17:420-4. (Level 2) 
7. Makino H, et al. Diabetes Care. 2007;30:1577-8. (Level 2)

8. Parving HH, et al. N Engl J Med. 2001;345:870-8. (Level 2)

9. Mauer M, et al. N Engl J Med. 2009;361:40-51. (Level 2)

10. Bilous R, et al. Ann Intern Med. 2009;151:11-20, W3-4. (Level 2)

11. Lewis EJ, et al. N Engl J Med. 1993;329:1456-62. (Level 2)

12. Brenner BM, et al. N Engl J Med. 2001;345:861-9. (Level 2)

13. Lewis EJ, et al. N Engl J Med. 2001;345:851-60. (Level 2)

14. Persson F, et al. Diabetes Care. 2009;32:1873-9. (Level 2)

15. Persson F, et al. Diabetologia. 2010;53:1576-80. (Level 2)

16. Parving HH, et al. N Engl J Med. 2008;358:2433-46. (Level 2)

17. Persson F, et al. Clin J Am Soc Nephrol. 2011;6:1025-31. (Level 2)

18. Ruggenenti P, et al. N Engl J Med. 2004;351:1941-51. (Level 2)

19. Agardh CD, et al. J Hum Hypertens. 1996;10:185-92. (Level 2)

20. Baba S, et al. Diabetes Res Clin Pract. 2001;54:191-201. (Level 2)

21. Velussi M, et al. Diabetes. 1996;45:216-22. (Level 2)

22. Barnett AH, et al. N Engl J Med. 2004;351:1952-61. (Level 2)

23. Bakris G, et al. Kidney Int. 2008;74:364-9. (Level 2)

24. Galle J, et al. Nephrol Dial Transplant. 2008;23:3174-83. (Level 2)

CQ7 Is antihypertensive therapy recommended to inhibit the involvement of CVD in diabetic patients with CKD?

We recommend antihypertensive therapy to prevent the involvement of CVD in diabetic patients with CKD. (Grade B)

Diabetes and hypertension are risk factors for CVD as well as dyslipidemia, obesity and smoking. Accordingly, the efficacy of antihypertensive therapy for CVD events should be evaluated.

There are many reports that antihypertensive therapy reduces the incidence of CVD events. Therefore antihypertensive therapy is recommended for diabetic patients with CKD. However, there are some reports that lowering the systolic blood pressure to less than $110 \mathrm{mmHg}$ raises the risk of death. Further studies are needed to determine the optimum target for blood pressure.

\section{Bibliography}

1. Heart Outcomes Prevention Evaluation Study Investigators. Lancet. 2000;355:253-9. (Level 2)

2. Berl T, et al. Ann Intern Med. 2003;138:542-9. (Level 2)

3. Imai E, et al. Diabetologia. 2011;54:2978-86. (Level 2)

4. Chalmers J, et al. J Hypertens. 2008;26(Suppl):S11-5. (Level 2)

5. Heerspink HJ, et al. Eur Heart J. 2010;31:2888-96. (Level 2)

6. Yusuf S, et al. N Engl J Med. 2008;358:1547-59. (Level 2)

7. Cushman WC, et al. N Engl J Med. 2010;362:1575-85. (Level 2)

8. Cooper-DeHoff RM, et al. JAMA. 2010;304:61-8. (Level 3)
CQ8 Are RAS inhibitors recommended

for normotensive diabetic patients with CKD?

We recommend RAS inhibitors to prevent kidney dysfunction in normotensive diabetic patients with CKD. (Grade B)

Currently, there is strong evidence that a RAS inhibitor is effective for diabetic patients with CKD. In normotensive type 1 diabetic patients, there is only little evidence that RAS inhibitors prevent progression of kidney dysfunction. In contrast to type 1 diabetic patients, there is some evidence that RAS inhibitors prevent the progression of kidney dysfunction in normotensive type 2 diabetic patients.

Moreover, there is some evidence that combinations of RAS inhibitors with other antihypertensive agents are also effective for preventing the progression of kidney dysfunction in normotensive type 2 diabetes.

There is some evidence that RAS inhibitors have renoprotective effects in type 2 diabetic patients, even among normotensive cases. However, the lowest target of BP is unknown and further investigations are needed to address this issue.

\section{Bibliography}

1. Mauer M, et al. N Engl J Med. 2009;361:40-50. (Level 2)

2. Ravid M, et al. Ann Intern Med. 1998;128:982-8. (Level 2)

3. Makino H, et al. Hypertens Res. 2008;31:657-64. (Level 2)

4. Jerums G, et al. Diabet Med. 2004;21:1192-9. (Level 2)

5. de Galan BE, et al. J Am Soc Nephrol. 2009;20:883-92. (Level 2)

6. Persson F, et al. Clin J Am Soc Nephrol. 2011;6:1025-31. (Level 2)

7. Cooper-DeHoff RM, et al. JAMA. 2010;304:61-8. (Level 3)

CQ9 Is a low protein diet recommended to suppress the progression of diabetic nephropathy?

We recommend a low protein diet, because an inhibitory effect on nephropathy can be expected. (Grade B)

The amount of protein restriction should be determined on the basis of risk to the individual patient's, pathophysiology and adherence.

In the development of progressive renal disease, including diabetic nephropathy, the activity of the underlying disease is important as a basic factor (blood glucose 
level in the case of diabetic nephropathy). In addition, hemodynamic and metabolic abnormalities are factors affecting the progression of renal injuries, and protein intake affects these factors. From the results of animal experiments, protein restriction has been found to exert a renoprotective effect through the improvement of glomerular hypertrophy, glomerular capillary resistance, and glomerular hypertension by improving abnormal metabolic factors and hemodynamics.

The effect on a low protein diet on suppressing the progression of diabetic nephropathy (especially in type 2 diabetes) is not clear. However, protein restriction can be expected to provide a renoprotective effect in diabetic nephropathy. Therefore, at the G3 stage of CKD, protein restriction of $0.8-1.0 \mathrm{~g} / \mathrm{kg}$ standard body weight/day is recommended, and at the G4 stage: $0.6-0.8 \mathrm{~g} / \mathrm{kg}$ standard body weight/day is recommended. The accumulation of additional evidence is required to make a recommendation on an advanced low protein diet $(<0.5 \mathrm{~g} / \mathrm{kg}$ standard body weight/day) and currently this should be determined by each individual patient's risk, pathophysiology and adherence.

\section{Bibliography}

1. Ciavarella A, et al. Diabetes Care. 1987;10:407-13. (Level 2)

2. Walker JD, et al. Lancet. 1989;2:1411-5. (Level 4)

3. Zeller K, et al. N Engl J Med. 1991;324:78-84. (Level 2)

4. Pedrini MT, et al. Ann Intern Med. 1996;124:627-32. (Level 1)

5. Kasiske BL, et al. Am J Kidney Dis. 1998;31:954-61. (Level 1)

6. Pan Y, et al. Am J Clin Nutr. 2008;88:660-6. (Level 1)

7. Koya D, et al. Diabetologia. 2009;52:2037-45. (Level 2)

CQ10 Is multifactorial intensive therapy recommended for suppressing the onset and progression of diabetic nephropathy?

We recommend multifactorial intensive therapy, including blood glucose, blood pressure and lipid control for suppressing the onset and progression of early diabetic nephropathy. (Grade B)

At the advanced stage of overt nephropathy, the effect of multifactorial intensive therapy for suppressing nephropathy is not clear.

The Steno-2 Study showed the effect of multifactorial intensive therapy, including blood glucose, blood pressure using RAS inhibitors and lipid control on the progression of nephropathy in microalbuminuric patients with type 2 diabetes. Therefore, multifactorial intensive therapy is recommended for suppressing the onset and progression of early diabetic nephropathy; however, it should be noted that this recommendation is based on a small RCT. In addition, the effect of multifactorial intensive therapy on the suppression of nephropathy is not clear at the advanced stage of overt nephropathy.

\section{Bibliography}

1. Gaede P, et al. N Engl J Med. 2003;348:383-93. (Level 2)

2. Gaede P, et al. N Engl J Med. 2008;358:580-91. (Level 2)

3. Tu ST, et al. Arch Intern Med. 2010;170:155-61. (Level 4)

CQ11 Is multifactorial intensive therapy recommended for suppressing the onset of CVD in diabetic nephropathy?

We recommend multifactorial intensive therapy, including blood glucose, blood pressure and lipid control for suppressing the onset of CVD in patients with diabetic nephropathy. (Grade B)

Diabetes increases the risk of developing both microvascular complications and CVD. Many patients who have diabetic nephropathy are complicated with hypertension and dyslipidemia and, therefore, are at an even greater risk of the involvement of CVD. The Steno-2 Study showed the effect of multifactorial intensive therapy, including blood glucose, blood pressure using RAS inhibitors and lipid control on the progression of nephropathy in type 2 diabetic patients with microalbuminuria. Therefore, multifactorial intensive therapy is recommended for suppressing the involvement of CVD in early diabetic nephropathy; however, it should be noted that this recommendation is based on a small RCT. In addition, the effect of multifactorial intensive therapy on the suppression of CVD is not clear at the advanced stage of overt nephropathy.

\section{Bibliography}

1. Gaede P, et al. N Engl J Med. 2003;348:383-93. (Level 2)

2. Gaede, P, et al. N Engl J Med. 2008; 58:580-91. (Level 2)

\section{Chapter 10: IgA nephropathy (IgAN)}

\section{Clinical outcomes}

\section{Clinical course and long-term outcomes}

In patients with $\operatorname{IgAN}$, progression to end-stage kidney disease (ESKD) is approximately 15 to 20 percent at 10 years and 40 percent at 20 years after diagnosis. 
When IgAN was described by Berger and Hinglais in 1968 , its prognosis was thought to be favorable. However, after 10- and 20-year outcomes were reported in many countries, including Japan, and ESKD was shown to occur in about 15 and $40 \%$ of cases, the prognosis could no longer be considered favorable. Among the results from Japan, Asaba et al. reported ESKD in $31 \%$ of patients after 7 years without treatment. Table 5 shows recent reports of renal survival at 10 years in various countries, as summarized by D'Amico.

2. Clinical predictors of progression

Clinical predictors of progression of $\operatorname{IgAN}$ include elevated serum creatinine at the time of diagnosis, persistent urinary protein excretion above $1 \mathrm{~g} / \mathrm{day}$, hypertension, severe glomerular sclerosis, and tubulointerstitial injuries.

In 2004, D'Amico reviewed the results of 23 major studies from 1984 to 2002 and indicated that severe proteinuria and hypertension at onset and during the course of observation, and elevated serum creatinine levels at onset, represent strong clinical predictors. His review also indicated that no history of macroscopic hematuria, male sex, and advanced age at onset are weak clinical predictors of poor prognosis.

With respect to proteinuria and hypertension, four recent studies have reported that mean urine protein level and mean blood pressure during the observation period are stronger risk factors than levels at the time of initial examination or renal biopsy.

3. Assessment of risk of progression

A scoring system to predict renal outcome has been established using a nationwide survey in Japan. The estimated risk of ESKD should be assessed using this scoring system. Even IgAN patients with benign presentation such as minimal proteinuria (less than $0.5 \mathrm{~g}$ /day), normal kidney function, and normal blood pressure, may show a slowly progressive course and develop renal failure by the time of a long-term follow-up.

In recent years, models to predict prognosis from the time of initial examination or renal biopsy have been developed with combinations of multiple risk factors for kidney failure, and are used to make 10 and 20 year prognostic predictions for IgAN. In 2005, Goto et al., using a Japanese IgAN patient database, conducted a survey of outcomes for 10 years. They then scored risk factors identified in multivariate analysis and predicted the incidence of ESKD from the total score (Tables 6, 7). In 2011 Bjørneklett et al. examined Goto et al.'s prognostic prediction model and confirmed its utility in 633 Norwegian patients with $\operatorname{IgAN}$.

Table 5 Renal survival of IgAN patients in the world

\begin{tabular}{|c|c|c|c|c|}
\hline Reporter & Report year & Patient number & $\begin{array}{l}\text { Average } \\
\text { observational } \\
\text { period(month) }\end{array}$ & $\begin{array}{l}10 \text {-year renal } \\
\text { survival }(\%)\end{array}$ \\
\hline \multicolumn{5}{|l|}{ Europe } \\
\hline D’Amico G (Italy) & 1986 & 365 & 79 & 85 \\
\hline Beukhof et al. (The Netherlands) & 1986 & 75 & 92 & $84^{*}$ \\
\hline Noel et al. (France) & 1987 & 280 & $>60$ & $85^{*}$ \\
\hline Velo et al. (Spain) & 1987 & 153 & $>60$ & $81 *$ \\
\hline Bogenschutz et al. (German) & 1990 & 239 & 59 & $81^{\$}$ \\
\hline Rekola et al. (Sweden) & 1990 & 209 & 76 & $83^{\#}$ \\
\hline Alamartine et al. (France) & 1991 & 282 & 96 & $94 *$ \\
\hline Johnston et al. (UK) & 1992 & 220 & 65 & $83^{\#}$ \\
\hline Payton et al. (UK) & 1988 & 67 & - & $77 *$ \\
\hline Manno et al. (Italy) ${ }^{4}$ & 2007 & 437 & 107 & $82^{\#}$ \\
\hline \multicolumn{5}{|l|}{ Australia } \\
\hline Nicolls et al. & 1984 & 244 & 60 & $87^{\#}$ \\
\hline Ibels et al. & 1994 & 121 & 107 & $93 *$ \\
\hline \multicolumn{5}{|l|}{ Asia } \\
\hline Woo et al. (Singapore) & 1986 & 151 & 65 & $91^{\#}$ \\
\hline Kusumoto et al. (Japan) & 1987 & 87 & 114 & $80 *$ \\
\hline
\end{tabular}


Table 5 continued

\begin{tabular}{lllll}
\hline Reporter & Report year & Patient number & $\begin{array}{l}\text { Average } \\
\text { observational } \\
\text { period(month) }\end{array}$ & $\begin{array}{l}10 \text {-year renal } \\
\text { survival }(\%)\end{array}$ \\
\hline Katafuchi et al. (Japan) & & & 48 & $74^{\#}$ \\
Yagame et al. (Japan) & 1994 & 225 & 110 & $87^{\#}$ \\
Koyama et al. (Japan) & 1996 & 206 & 142 & $85^{*}$ \\
Le et al. (Hong Kong) & 1997 & 448 & 88 & $82^{\#}$ \\
Lv J et al. (China) & 210 & 168 & 73 & $77^{\#}$ \\
Le W et al. (China) & 2008 & 204 & Median 5.4 years $(4.1-7.2)$ & $83^{\#}$ \\
North America & 2011 & 1,155 & $>60$ & $78^{*}$ \\
Wyatt et al. (USA) & & & 45 & $67^{\#}$ \\
Radford et al. (USA) & 1984 & 58 & 718 & $57^{\#}$ \\
Haas (USA) & 1997 & 148 & 70 & $65^{*}$ \\
Bartosik et al. (Canada) & 1997 & 298 & & \\
\hline
\end{tabular}

Modified Table 1 in Bibliography No. 22 with other reports

* From the time of diagnosis

$\$$ Not specified

\# From the time of biopsy

Table 6 Scores of individual prognostic factors to estimate the 10-year risk of ESKD

\begin{tabular}{lc}
\hline Male sex & 6 \\
Age $<30$ years & 12 \\
Systolic blood pressure $(\mathrm{mmHg})$ \\
$<130$ & 0 \\
$131-160$ & 4 \\
$>160$ & 11 \\
Urine protein & \\
,- \pm & 0 \\
+ & 12 \\
$2+$ & 21 \\
$3+$ & 25 \\
Mild haematuria & \\
(RBC1 29/HPF) & 8 \\
Serum albumin & \\
$<4.0$ g/dL & 7 \\
eGFR & \\
$>90$ & 0 \\
$60-90$ & 7 \\
$30-60$ & 22 \\
$15-30$ & 42 \\
$<15$ & 66 \\
(15 & 5
\end{tabular}

Cited from Bibliography No. 16

Histological grade III or IV 5

\section{Bibliography}

1. McEnery PT, et al. Perspect Hephrol Hypertens. 19731;1:305-20. (Level 4)

2. Chauveau D, et al. Contrib Nephlol. 1993;104:1-5. (Level 4)

3. Koyama A, et al. Am J Kidney Dis. 1997;29:526-32. (Level 4)

4. Manno C, et al. Am J Kidney Dis. 2007;49:763-75. (Level 4)

5. Le W, et al. Nephrol Dial Transplant. 2012;27:1479-85. (Level 4)
Table 7 Estimated 10-year risk of ESRD by total score

\begin{tabular}{lc}
\hline Total score & Estimated 10-year risk of ESKD $(\%)$ \\
\hline $0-26$ & $0-1$ \\
$27-43$ & $1-5$ \\
$44-50$ & $5-10$ \\
$51-58$ & $10-20$ \\
$59-63$ & $20-30$ \\
$64-70$ & $30-50$ \\
$71-75$ & $50-70$ \\
$76-82$ & $70-90$ \\
$83-140$ & $90-100$
\end{tabular}

Cited from Bibliography No. 16

6. Asaba K, et al. Intern Med. 2009;48:883-90. (Level 4)

7. Kobayashi Y, et al. Nephrology. 1997;3:35-40. (Level 4)

8. Bartosik LP, et al. Am J Kidney Dis. 2001;38:728-35. (Level 4)

9. Reich HN, et al. J Am Soc Nephrol. 2007;18:3177-83. (Level 4)

10. Hwang HS, et al. Nephrology (Carlton) 2010;15:236-41. (Level4)

11. Magistroni R, et al. J Nephrol. 2006;19:32-40. (Level 4)

12. Alamartine E, et al. Clin J Am Soc Nephrol. 2011;6:2384-8. (Level 4)

13. Working Group of the International IgA Nephropathy Network and the Renal Pathology Society. Kidney Int. 2009;76:534-45. (Level 4)

14. Kang SH, et al. Nephrol Dial Transplant. 2012;27:252-8. (Level 4)

15. Katafuchi R, et al. Clin J Am Soc Nephrol. 2011;6:2806-13. (Level 4)

16. Goto M, et al. Nephrol Dial Transplant. 2009;24:3068-74. (Level 4)

17. Bjørneklett R, et al. Nephrol Dial Transplant. 2012;27:1485-91. (Level 4) 
18. Berthoux F, et al. J Am Soc Nephrol. 2011;22:752-61. (Level 4)

19. Szeto CC, et al. Am J Med. 2001;110:434-7. (Level 4)

20. Shen P, et al. Neth J Med. 2008;66:242-7. (Level 4)

21. Lv J, et al. Nephrology (Carlton). 2008;13:242-6. (Level 4)

22. D’Amico G. Semin Nephrol. 2004;24:179-96.

\section{Treatment of IgAN}

Major potential treatment modalities for adult IgAN in Japan are RAS inhibitors, corticosteroids, nonsteroidal immunosuppressive agents, antiplatelet agents, n-3 fatty acids (fish oil) and tonsillectomy (with corticosteroid pulse therapy) (Figs. 2, 3). To suppress IgAN progression, these treatments should be considered based on renal function, urinary protein, age, renal histopathological findings, and so on. Interventions to optimize blood pressure, salt intake, lipid and glucose metabolism, body weight, smoking habits, and so on should be considered, if necessary. (Fig. 4)

We evaluated the effectiveness of interventions in slowing the progression of renal dysfunction and decreasing urine protein based mainly on results of reported randomized parallel-group trials (Figs. 2, 3) and made suggestions about treatment options (Fig. 4).

1. Adult $\operatorname{IgAN}$ with urine protein $\geq 1.00 \mathrm{~g} /$ day and (CKD) stage G1-2 First-line therapy: RASinhibitors and/or steroid therapy. Second-line therapy: Immunosuppressive agents, antiplatelet agents, tonsillectomy (+steroid pulse therapy), fish oil, etc.

2. Adult IgAN with urine protein $\geq 1.00 \mathrm{~g} /$ day and $\mathrm{CKD}$ stage G3 First-line therapy: RAS inhibitors. Second-line therapy: Steroid therapy, immunosuppressive agents, antiplatelet agents, tonsillectomy ( + steroid pulse therapy), fish oil, etc.

3. Adult IgAN with urine protein $0.50-0.99 \mathrm{~g} /$ day and CKD stage G1-3. Intervention should be considered because urine protein of $0.50-0.99 \mathrm{~g} /$ day has been reported to be a possible risk factor related to poor renal prognosis and urine protein should not be allowed to increase to $\geq 1.00 \mathrm{~g} / \mathrm{day}$, which is clearly a risk factor for unfavorable renal prognosis.

4. Adult IgAN with urine protein $<0.50 \mathrm{~g} /$ day and CKD stage G1-2. Renal function outcome in IgAN with urine protein of $<0.50 \mathrm{~g} / \mathrm{day}$ and CKD stage $\mathrm{G} 1-3$ is predicted to be favorable.

5. Adult IgAN with urine protein $<1.00 \mathrm{~g} /$ day and CKD stage G3 or G4-5. Treatment interventions in accordance with the evidence-based CKD guideline 2013 are appropriate (Fig. 4).
CQ1 Are antiplatelet agents and anticoagulants recommended for decreasing urinary protein and preserving renal function in patients with $\operatorname{IgAN}$ ?

We suggest that perhaps dipyridamole may be used because of its potential effect of lowering proteinuria and preventing renal impairment. (Grade C1) Similarly, we suggest that perhaps dilazep hydrochloride may be used because of its potential effect of lowering proteinuria. (Grade $\mathrm{C} 1$ )

In the 1980s, a multi-center, randomized, double-blinded controlled trial with dipyridamole and dilazep hydrochloride for chronic glomerulonephritis, including IgAN, was conducted in Japan. This study showed that antiplatelet agents were effective in reducing urine protein levels. However, since the report was not published in an English-language journal, it did not draw international attention.

Systematic reviews evaluating the effect of dipyridamole and dilazep hydrochloride in slowing the progression of renal dysfunction and decreasing urine protein in IgAN have not been able to produce solid conclusions, since there are too few randomized parallel-group trials. Smallscale randomized parallel-group trials have suggested the possibility that these drugs have an effect in decreasing urine protein, but these studies were not of high quality.

\section{Bibliography}

1. Taji Y, et al. Clin Exp Nephrol. 2006;10:268-73. (Level 3)

2. Liu XJ, et al. Intern Med. 2011;50:2503-10. (Level 1)

3. Chan MK, et al. Am J Kidney Dis. 1987;9:417-21. (Level 2)

4. Lee GSL, et al. Nephrology. 1997;3:117-21. (Level 2)

5. Camara S, et al. Nephron. 1991;58:13-6. (Level 2)

6. Cheng IKP, et al. Nephrology. 1998;4:19-26. (Level 2)

CQ2 Are RAS inhibitors recommended for decreasing urinary protein and preserving renal function in patients with IgAN?

We recommend treatment with RAS inhibitors for patients with proteinuria $\geq 1 \mathrm{~g} /$ day and eGFR $\geq 30 \mathrm{ml} / \mathrm{min} / 1.73 \mathrm{~m}^{2}$ (CKD G1 to G3b) because of their effect of preservation of renal function. (Grade A)

We suggest treatment with RAS inhibitors if proteinuria is between 0.5 to $1.0 \mathrm{~g} /$ day because of their antiproteinuric effect. (Grade C1)

A number of randomized parallel-group trials have shown that RAS inhibitors for IgAN with urine protein 


$\begin{array}{ll}\begin{array}{l}\text { Author, publication } \\ \text { year, country, number } \\ \text { of institutions }\end{array} & \begin{array}{l}\text { OIntervention group (maximum dose/day, } \\ \left.\text { administration period }{ }^{2}\right)\end{array} \\ & \text { OControl group }\end{array}$

Fig. 2 The summary of randomized controlled trials of corticosteroids and immunosuppressive agents in adult patients with IgAN. $A Z A$ azathioprine, CPA cyclophosphamide, CyA ciclosporin, ITT intention to treat, $M M F$ mycophenolate mofetil, $m P S L$ methylprednisolone, $M Z R$ mizoribine, $P P$ pet protocol, $P S L$ prednisolone, $P S N$

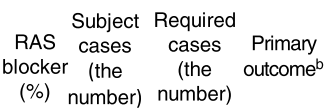

Pre-intervention findings Urinary protein

$\begin{array}{rrrcrr} & & & & & \\ 35 & 17 & & & & \\ 44 & 18 & - & & 2 & \text { ITT } \\ 100 & 33 & 67 & \begin{array}{c}\text { Serum Cr } \\ \text { Cr }\end{array} & & \\ 100 & 30 & 67 & \geq 150 \% & \text { ITT } \\ 100 & 48 & 60 & \text { Serum Cr } & & \\ 100 & 49 & 60 & \geq 200 \% & 5 & \text { ITT }\end{array}$

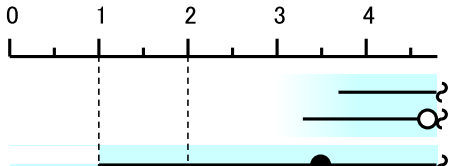

eGFR
$<60 \%$

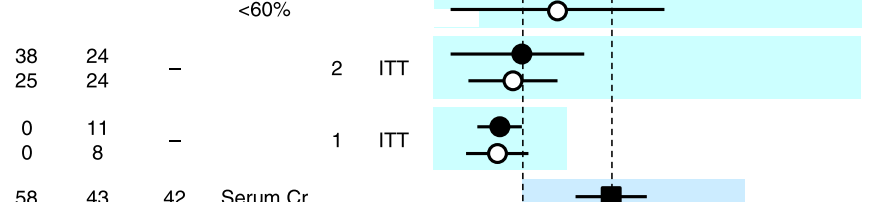

$58 \quad 43 \quad 42 \quad$ Serum $\mathrm{Cr}$

$42 \quad \geq 150 \%$

$\begin{array}{llll}0 & 25 & - & 2\end{array}$

$26 \quad 19$

$26 \quad 19$

89

$\begin{array}{cccccc}92 & 106 & 173 & \geq 150 \% & 5 & \text { ITT } \\ & 21 & & & & \\ & 20 & - & & - & \text { ITT }\end{array}$

$\begin{array}{ccccc}- & 9 & & & \\ - & 10 & - & 0.5 & \text { ITT }\end{array}$

$100 \quad 17 \quad 50 \quad$ Serum $\mathrm{Cr}$

$\begin{array}{cccccc}100 & 17 & 50 & \text { Serum } \mathrm{Cr} & 2 & \text { ITT } \\ 100 & 15 & 50 & \geq 150 \% & & \end{array}$

$\begin{array}{lllll}100 & 15 & 50 \quad \geq 150 \% \quad 2 & \end{array}$

$\begin{array}{llllll}100 & 20 & 20 & \text { Urinary } & & \\ 100 & 20 & 20 & \text { protein } & 1.4 & \text { ITT }\end{array}$

$\begin{array}{llll}100 \quad 21 \quad-\quad 3 & 3\end{array}$

$100 \quad 13$

100
0

0
100 


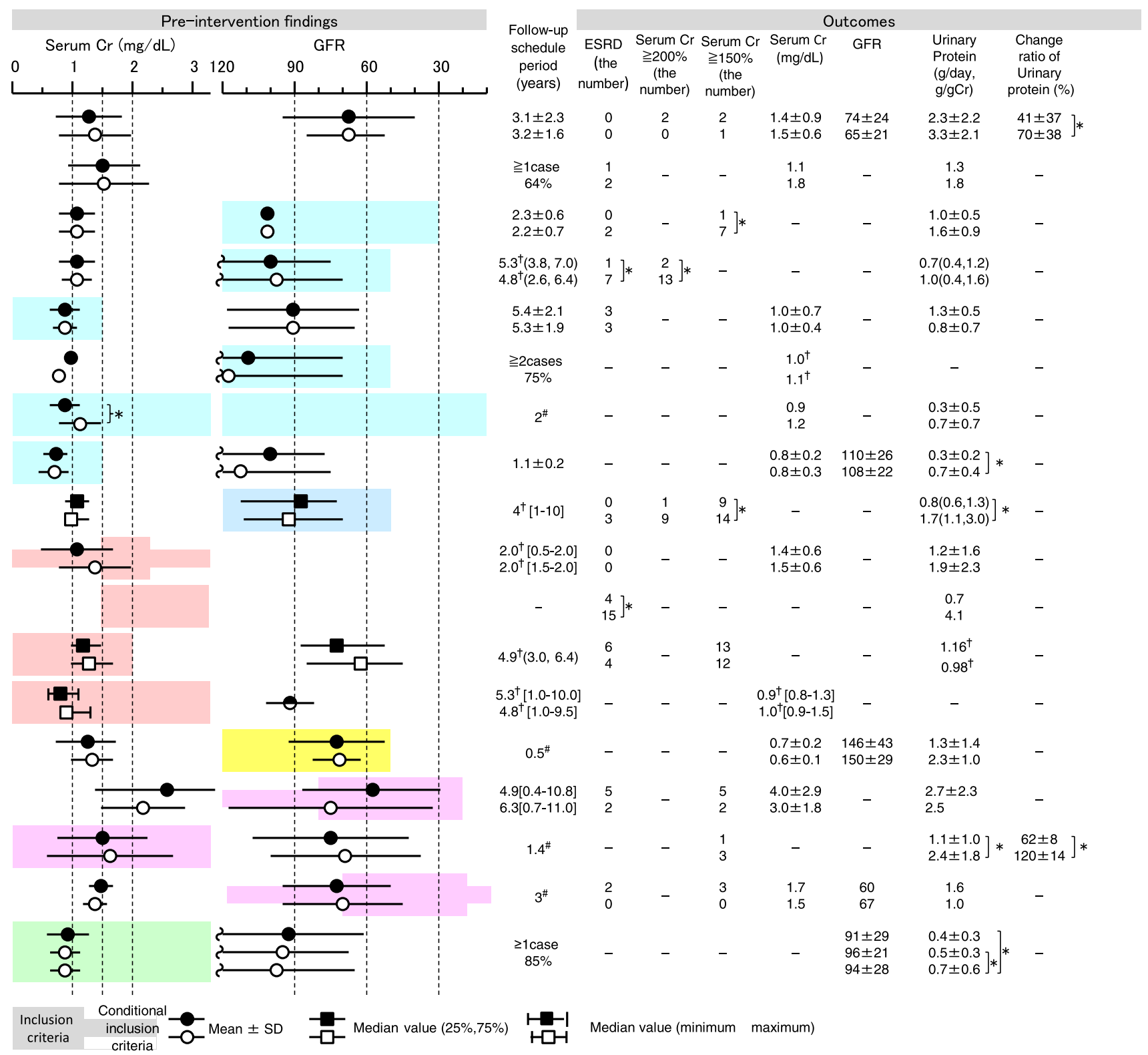

Fig. 2 continued 


\begin{tabular}{|c|c|c|}
\hline & $\begin{array}{l}\text { Author, publication year, } \\
\text { country, number of } \\
\text { institutions }\end{array}$ & $\begin{array}{l}\text { Intervention group (maximum dose/day, } \\
\text { administration period }{ }^{a, b} \\
\text { OControl group }\end{array}$ \\
\hline & Ruggenenti 2000 & $\begin{array}{l}\text { Ramipril (5mg) } \\
\text { OPlacebo }\end{array}$ \\
\hline & Woo 2000 & -Enalapril $(10 \mathrm{mg}) / \mathrm{losartan}(100 \mathrm{mg})$ \\
\hline & Singapore, 1 institution & OAntihypertensive agents except RAS blockers \\
\hline$\frac{0}{\pi}$ & Woo 2007 & Enalapril (10mg)/losartan (100mg) \\
\hline 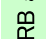 & Singapore, 1 institution & OAntihypertensive agents except RAS blockers \\
\hline to & Park 2003 & - Losartan (50mg) \\
\hline$\frac{0}{\frac{0}{0}}$ & $\begin{array}{l}\text { Li } 2006 \\
\text { China (Hong Kong), } \\
5 \text { institutions }\end{array}$ & $\begin{array}{l}\text { OValsartan (160mg) } \\
\text { OPlacebo }\end{array}$ \\
\hline 童 & $\begin{array}{l}\text { Praga } 2003 \\
\text { Spain, } 1 \text { institution }\end{array}$ & $\begin{array}{l}\text { OEnalapril (40mg) } \\
\text { OAntihypertensive agents except RAS blockers }\end{array}$ \\
\hline 岂 & $\begin{array}{l}\text { Nakamura } 2000 \\
\text { Japan, } 3 \text { institutions }\end{array}$ & $\begin{array}{l}\text { Trandolapril }(2 \mathrm{mg}) \\
\text { OCandesartan }(8 \mathrm{mg}) \\
\text { OVerapamil }(120 \mathrm{mg}) \\
\text { OPlacebo }\end{array}$ \\
\hline & $\begin{array}{l}\text { Coppo } 2007 \\
5 \text { European countries, } \\
23 \text { institutions }\end{array}$ & $\begin{array}{l}\text { Benazepril }(0.2 \mathrm{mg} / \mathrm{kg}) \\
\text { OPlacebo }\end{array}$ \\
\hline$\frac{0}{3} \frac{0}{0}$ & $\begin{array}{l}\text { Nakamura } 2007 \\
\text { Japan, } 4 \text { institutions }\end{array}$ & $\begin{array}{l}\text { Temocapril }(2 \mathrm{mg})+\text { olmesartan }(10 \mathrm{mg}) \\
\text { OTemocapril }(2 \mathrm{mg}) \\
\text { OOlmesartan }(10 \mathrm{mg})\end{array}$ \\
\hline है & $\begin{array}{l}\text { Horita } 2004 \\
\text { Japan, } 1 \text { institution }\end{array}$ & $\begin{array}{l}\text { OTemocapril }(1 \mathrm{mg})+\text { losartan }(12.5 \mathrm{mg}) \\
\text { OTemocapril }(1 \mathrm{mg}) \\
\text { OLosartan }(12.5 \mathrm{mg})\end{array}$ \\
\hline & $\begin{array}{l}\text { Tojo } 1987 \\
\text { Japan, } 84 \text { institutions }\end{array}$ & $\begin{array}{l}\text { ODipyridamole (300mg) } \\
\text { OPlacebo }\end{array}$ \\
\hline 艺 & $\begin{array}{l}\text { Chan } 1987 \\
\text { China (Hong Kong), } \\
1 \text { institution }\end{array}$ & $\begin{array}{l}\text { Dipyridamole }(225 \mathrm{mg})+\text { aspirin }(650 \mathrm{mg}) \\
\text { OVitamin B }\end{array}$ \\
\hline 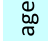 & Camara 1991 & Dipyridamole (300mg) \\
\hline$\frac{\omega}{0}$ & Spain, 2 institutions & OPlacebo \\
\hline$\underset{\substack{0 \\
0}}{\underline{0}}$ & Lee 1997 & Dipyridamole $(225 \mathrm{mg})+$ warfarin \\
\hline 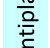 & Singapore, 1 institution & ONo dipyridamole + warfarin \\
\hline & $\begin{array}{l}\text { Tojo } 1986 \\
\text { Japan, } 67 \text { institutions }\end{array}$ & $\begin{array}{l}\text { Dilazep (300mg) } \\
\text { OPlacebo }\end{array}$ \\
\hline & Cheng 1998 & -Ticlopidine $(500 \mathrm{mg})+$ captopril $(150 \mathrm{mg})$ \\
\hline & Singapore, 1 institution & OCaptopril (13.5-150mg) \\
\hline & $\begin{array}{l}\text { Donadio 1994, } 1999 \\
\text { US, } 21 \text { institutions }\end{array}$ & $\begin{array}{l}\text { OEPA }(1.9 \mathrm{~g})+\text { DHA }(1.4 \mathrm{~g}) \\
\text { OPlacebo }\end{array}$ \\
\hline & Alexopoulos 2004 & -EPA $(0.9 \mathrm{~g})+\mathrm{DHA}(0.6 \mathrm{~g})$ \\
\hline & Greece, 1 institution & ONo EPA + DHA \\
\hline & Pettersson 1994 & OEPA $(3.3 \mathrm{~g})+\mathrm{DHA}(1.8 \mathrm{~g})$ \\
\hline & & \\
\hline & Bennett 1989 & OEPA $(1.8 \mathrm{~g})+\mathrm{DHA}(1.2 \mathrm{~g})$ \\
\hline 汇 & US, 1 institution & ONo EPA + DHA \\
\hline & $\begin{array}{l}\text { Hogg } 2006 \\
\text { US } 37 \text { institutions }\end{array}$ & OEPA $(1.9 \mathrm{~g})+\mathrm{DHA}(1.5 \mathrm{~g})$ \\
\hline & & \\
\hline & $\begin{array}{l}\text { Ferraro } 2009 \\
\text { Italy, } 1 \text { institution }\end{array}$ & $\begin{array}{l}\text { OEPA + DHA }(2.6 \mathrm{~g})+\text { RAS blocker } \\
\text { ORAS blocker }\end{array}$ \\
\hline & $\begin{array}{l}\text { Donadio } 2001 \\
\text { US, } 14 \text { institutions }\end{array}$ & $\begin{array}{l}\text { OHigh-dose EPA }(3.8 \mathrm{~g})+\text { DHA }(2.9 \mathrm{~g}) \\
\text { OLow-dose EPA }(1.9 \mathrm{~g})+\text { DHA }(1.5 \mathrm{~g})\end{array}$ \\
\hline
\end{tabular}

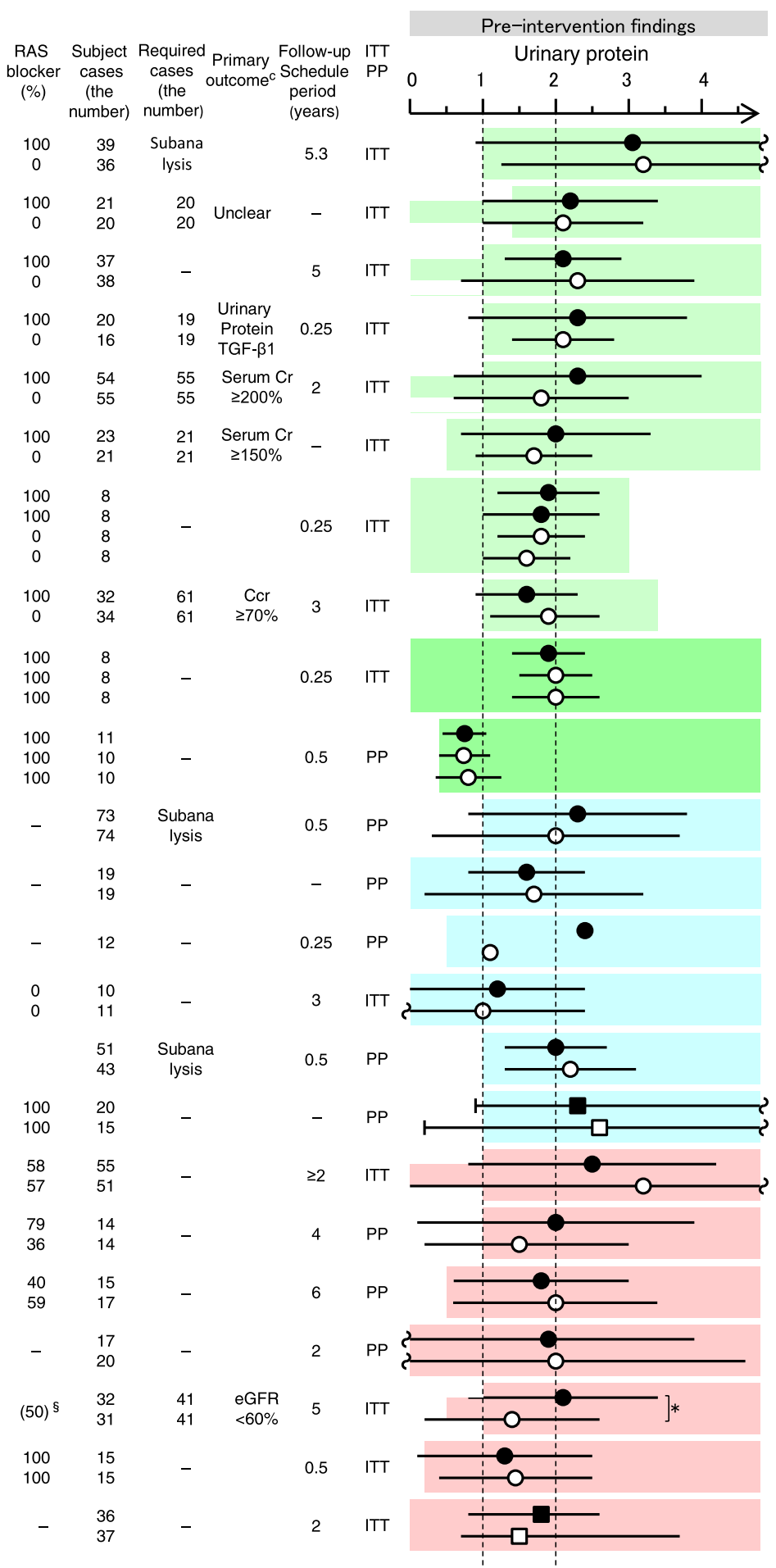

Fig. 3 Summary of randomized controlled trials of RAS inhibitors, antiplatelet agents, and fish oils in adult patients with IgAN. EPA eicosapentaenoic acid, DHA docosahexaenoic acid, ITT intention to treat, NS not significant, $P P$ pet protocol, $S I$ selectivity index. Mean $\pm \mathrm{SD}$, median value $(25 \%, 75 \%)$, mean or median value (minimum - maximum). No statement, $* p<0.05,{ }^{\S}$ pre-intervention medication rate. ${ }^{\#}$ Follow-up schedule period, ${ }^{\dagger}$ median value, ${ }^{a}$ only

when the intervention period is limited. ${ }^{\mathrm{b}}$ The domestically approved maximum dose of antihypertensive agents (mg)/US JNC7 recommendation dose: amlodipine (10/10), enalapril (10/40), olmesartan (40/40), captopril (150/100), candesartan (12/32), temocapril (4/-), trandolapril (2/4), valsartan (160/320), benazepril (10/40), verapamil (360/360), tamipril $(-/ 10)$, losartan $(100 / 100),{ }^{c}$ only when the number of required cases is calculated 


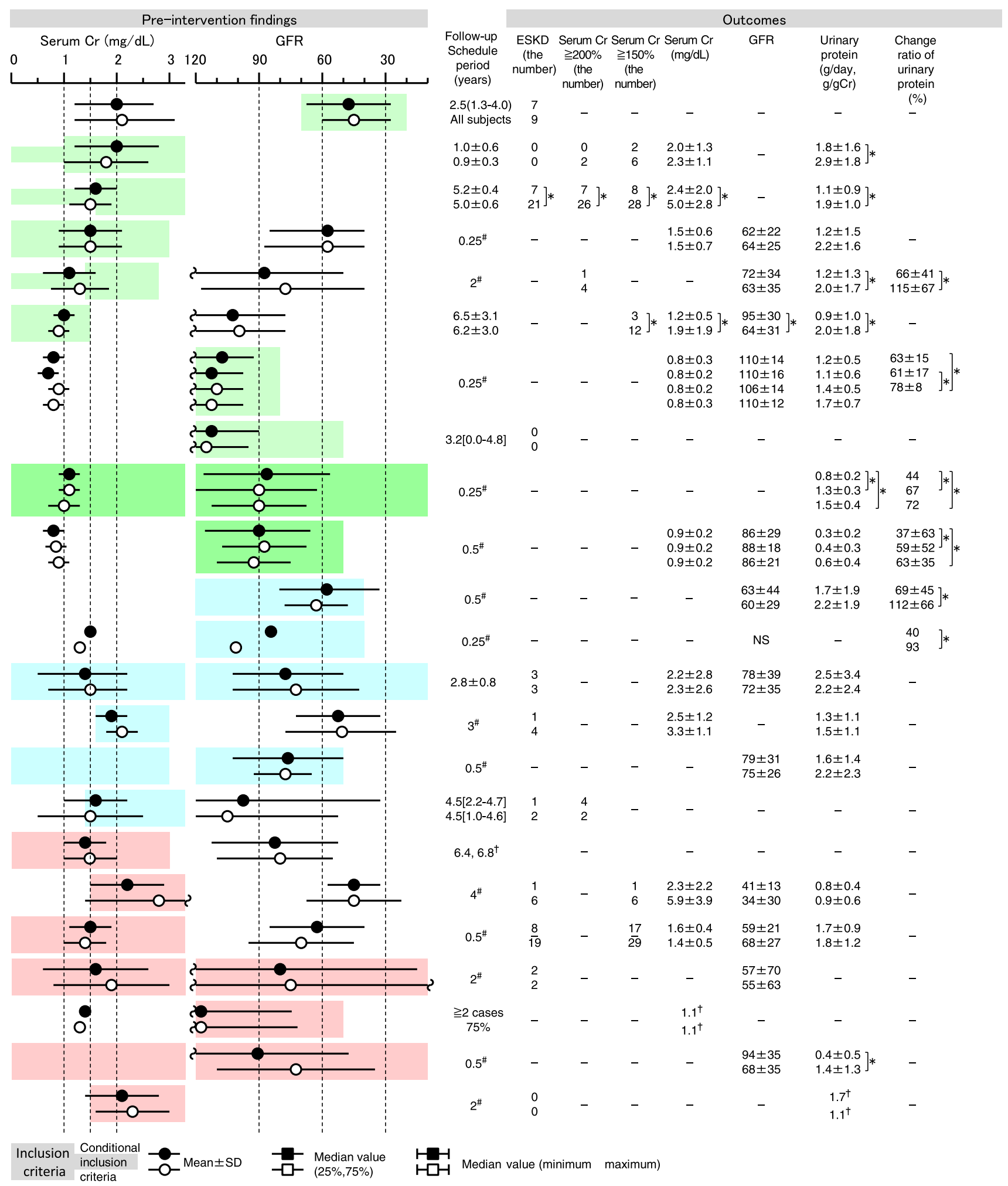

Fig. 3 continued 
$\geq 1 \mathrm{~g} /$ day and CKD stage G1-3 are effective in slowing the progression of renal dysfunction and decreasing urine protein levels. RAS inhibitors are thus determined to have a recommendation grade of A for IgAN with urine protein $\geq 1 \mathrm{~g} /$ day and CKD stage G1-3. By contrast, among randomized parallel-group trials investigating the efficacy of RAS inhibitors mainly for IgAN with urine protein of $0.5-1.0 \mathrm{~g} /$ day, the only report to show that increased doses of RAS inhibitors enhanced the urine protein-decreasing effect was that of Horita (2004). Therefore, RAS inhibitors for IgAN with urine protein of $0.5-1.0 \mathrm{~g} /$ day are determined to have a recommendation grade of $\mathrm{C} 1$.

\section{Bibliography}

1. Cheng J, et al. Int J Clin Pract. 2009;63:880-8. (Level 1)

2. Reid S, et al. Cochrane Database Syst Rev. 2011;3:CD003962. (Level 1)

3. Praga M, et al. J Am Soc Nephrol. 2003;14:1578-83. (Level 2)

4. Woo KT, et al. Cell Mol Immunol. 2007;4:227-32. (Level 2)

5. Ruggenenti P, et al. Am J Kidney Dis. 2000;35:1155-65. (Level 2)

6. Woo KT, et al. Kidney Int. 2000;58:2485-91. (Level 2)

7. Park HC, et al. Nephrol Dial Transplant. 2003;18:1115-21. (Level 2)

8. Li PK, et al. Am J Kidney Dis. 2006;47:751-60. (Level 2)

9. Nakamura T, et al. Am J Nephrol. 2000;20:373-9. (Level 2)

10. Coppo R, et al. J Am Soc Nephrol. 2007;18:1880-8. (Level 2)

11. Horita Y, et al. Hypertens Res. 2004;27:963-70. (Level 2)

12. Nakamura T, et al. Am J Hypertens. 2007;20:1195-201. (Level 2)

CQ3 Are corticosteroids recommended for decreasing urinary protein and preserving renal function in patients with IgAN?

We recommend that patients with proteinuria $\geq 1 \mathrm{~g} /$ day and eGFR $\geq 60 \mathrm{ml} / \mathrm{min} / 1.73 \mathrm{~m}^{2}$ (CKD G1 to G2) receive a 6-month course of high-dose oral corticosteroids therapy (6-month regime of oral prednisone starting with 0.8 to $1 \mathrm{mg} / \mathrm{kg} / \mathrm{day}$ for 2 months and then reduced gradually for the next 4 months). (Grade B)

We recommend that patients with proteinuria $\geq 1 \mathrm{~g} /$ day and eGFR $\geq 60 \mathrm{ml} / \mathrm{min} / 1.73 \mathrm{~m}^{2}$ (CKD G1 to G2) receive high-dose pulse corticosteroids therapy (i.v. bolus injections of $1 \mathrm{~g}$ methylprednisolone for 3 days each at months 1,3 , and 5 , followed by $0.5 \mathrm{mg} / \mathrm{kg}$ oral prednisone on alternate days for 6 months). (Grade B)

We tentatively suggest using corticosteroids for patients with proteinuria less than $1 \mathrm{~g} /$ day and eGFR $\geq 60 \mathrm{ml} / \mathrm{min} / 1.73 \mathrm{~m}^{2}$ (CKD G1 to G2) because of their antiproteinuric effect. (Grade $\mathrm{C} 1$ )
Short-term, high-dose, oral steroid therapy and steroid pulse therapy for IgAN with urine protein of $\geq 1 \mathrm{~g} /$ day and CKD stage G1-2 have been shown to be effective in slowing the progression of renal dysfunction and decreasing urine protein in a small number of randomized parallelgroup trials. The recommendation grade for both of these therapies is thus determined to be B. However, steroid therapy for IgAN with urine protein $0.5-1.0 \mathrm{~g} /$ day does not have a confirmed effect in slowing the progression of renal dysfunction, and its effect in decreasing urine protein has been confirmed in only some small-scale trials. The recommendation grade is therefore determined to be $\mathrm{C} 1$.

\section{Bibliography}

1. Lv J. J Am Soc Nephrol. 2012;23:1108-16. (Level 1)

2. Zhou YH, et al. PLoS One. 2011;6:e18788. (Level 4)

3. Cheng J. Am J Nephrol. 2009;30:315-22. (Level 1)

4. Samuels JA, et al. Cochrane Database Syst Rev. 2003:CD003965. (Level 3)

5. Lv J, et al. Am J Kidney Dis. 2009;53:26-32. (Level 2)

6. Manno C, et al. Nephrol Dial Transplant. 2009;24:3694-701. (Level 2)

7. Pozzi C, et al. Lancet. 1999;353:883-7. (Level 2)

8. Pozzi C, et al. J Am Soc Nephrol. 2004;15:157-63. (Level 2)

9. Lai KN, et al. Clin Nephrol. 1986;26:174-80. (Level 2)

10. Julian BA, et al. Contrib Nephrol. 1993;104:198-206. (Level 2)

11. Katafuchi R, et al. Am J Kidney Dis. 2003;41:972-83. (Level 2)

12. Hogg RJ. Clin J Am Soc Nephrol. 2006;1:467-74. (Level 2)

13. Koike M, et al. Clin Exp Nephrol. 2008;12:250-5. (Level 2)

14. Shoji T, et al. Am J Kidney Dis. 2000;35:194-201. (Level 2)

CQ4 Is tonsillectomy recommended for decreasing urinary protein and preserving renal function in patients with IgAN?

We tentatively suggest that tonsillectomy combined with high-dose pulse corticosteroids therapy may be indicated for patients with IgAN because of its potential effect of preservation of renal function. (Grade C1)

In Japan, tonsillectomy plus steroid pulse therapy is widely used. However, no clear consensus has yet been reached on its effect in slowing the progression of renal dysfunction and the indications for this treatment.

Combination therapy with tonsillectomy and steroid pulse therapy for IgAN, in comparison with steroid pulse therapy alone, has been reported from a small number of randomized parallel-group trials and cohort studies to enhance the effect in decreasing urine protein, and therapeutic options should be investigated. At present, however, there do not seem to be any therapies that should be more 


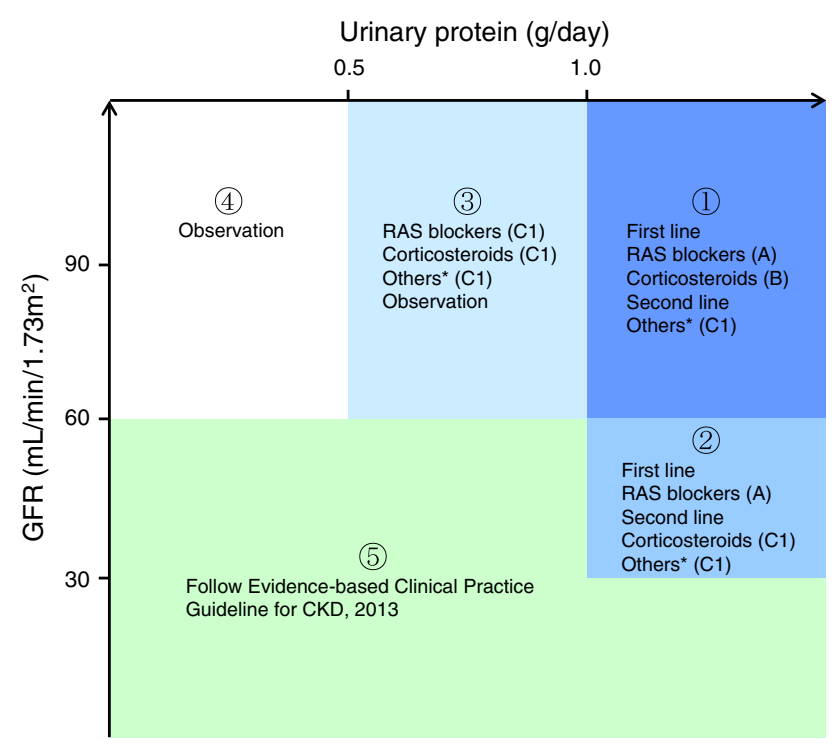

Fig. 4 An outline of treatment of IgAN in adults: focused on prevention of renal dysfunction (based on randomized controlled trials for IgAN). Choice of treatment should be carefully considered based on renal function, the amount of proteinuria, pathological findings, age, and other clinical findings. Others: tonsillectomy (combined with high-dose pulse corticosteroid therapy), immunosuppressive agents, antiplatelet agents, and n-3 fatty acids (fish oil)

strongly recommended than steroid therapy or RAS inhibitors.

\section{Bibliography}

1. Wang Y, et al. Nephrol Dial Transplant. 2011;26:1923-31. (Level 1)

2. Komatsu H, et al. Clin J Am Soc Nephrol. 2008;3:1301-7. (Level 3)

3. Hotta O, et al. Am J Kidney Dis. 2001;38:736-43. (Level 4)

4. Kawaguchi T, et al. Nephrology. 2010;15:116-23. (Level 4)

5. Sato M, et al. Nephron Clin Pract. 2003;93:c137-45. (Level 4)

6. Xie Y, et al. Kidney Int. 2003;63:1861-7. (Level 4)

7. Maeda I, et al. Nephrol Dial Transplant. 2012;27:2806-13. (Level 4)

8. Chen Y, et al. Am J Nephrol. 2007;27:170-5. (Level 4)

CQ5 Are immunosuppressive agents recommended for reducing urinary protein and preserving renal function in patients with IgAN?

We tentatively suggest that immunosuppressive agents (cyclophosphamide, azathioprine, cyclosporine, MMF, and mizoribine) may be expected to improve renal outcome. (Grade $\mathrm{C} 1$ )

It is possible that renal prognosis in $\operatorname{IgAN}$ can be improved with addition of immunosuppressants in combination with steroids, which plays a central role in the treatment of IgAN.
A very small number of randomized parallel-group trials have investigated the renoprotective effects of cyclophosphamide, azathioprine, cyclosporine, mycophenolate mofetil, and mizoribine for IgAN, nearly all of which were small-scale trials with low power. Reaching any solid conclusions is currently difficult, but results suggesting effects in decreasing urine protein and slowing the progression of renal dysfunction have been reported, so the recommendation grade for all of these drugs is $\mathrm{C} 1$. However, azathioprine in combination with steroid therapy may increase the incidence of adverse effects, and its use should therefore be considered carefully.

\section{Bibliography}

1. Walker RG, et al. Clin Nephrol. 1990;34:103-7. (Level 2)

2. Ballardie FW, et al. J Am Soc Nephrol. 2002;13:142-8. (Level 2)

3. Pozzi C, et al. J Am Soc Nephrol. 2010;21:1783-90. (Level 2)

4. Harmankaya O, et al. Int Urol Nephrol. 2002;33:167-71. (Level 2)

5. Lai KN, et al. BMJ. 1987;295:1165-8. (Level 2)

6. Frisch G, et al. Nephrol Dial Transplant. 2005;20:2139-45. (Level 2)

7. Tang S, et al. Kidney Int. 2005;68:802-12. (Level 2)

8. Maes BD, et al. Kidney Int. 2004;65:1842-9. (Level 2)

9. Xu G, et al. Am J Nephrol. 2009;29:362-7. (Level 1)

10. Xie Y, et al. Am J Med Sci. 2011;341:367-72. (Level 2)

\section{Chapter 11: Nephrotic syndrome}

CQ1 Is cancer screening recommended for patients with membranous nephropathy?

The incidence of cancer in Asian and Japanese patients with membranous nephropathy is lower than that of other races.

We recommend that the need for cancer screening be determined on an individual basis after considering the condition of each patient with membranous nephropathy.

Cancer is one of the leading causes of secondary membranous nephropathy. In western countries, about $7-10 \%$ of patients with membranous nephropathy have been complicated with cancer. In Japan, however, the renal biopsy registry shows that less than $1.0 \%$ of membranous nephropathy patients have been complicated with cancer, especially with only two cases with solid tumors.

From these data, the complication rate for cancer in Japanese patients with membranous nephropathy is lower than that of western countries. It remains unclear whether the cancer is more complex in patients with membranous nephropathy than in the general population in Japan. 
Further study is needed to reveal the relationship between membranous nephropathy and cancer.

\section{Bibliography}

1. Burstein DM, et al. Am J Kidney Dis. 1993;22:5-10. (Level 4)

2. Lefaucheur C, et al. Kidney Int. 2006;70:1510-7. (Level 4)

3. Bjorneklett R, et al. Am J Kidney Dis. 2007;50:396-403. (Level 4)

4. Zeng CH, et al. Am J Kidney Dis. 2008;52:691-8. (Level 4)

5. Yokoyama H, et al. Clin Exp Nephrol. 2012;16:557-63. (Level 4)

CQ2 Is cyclophosphamide with corticosteroid recommended for the treatment of idiopathic membranous nephropathy?

We recommend cyclophosphamide combined with corticosteroid for the treatment of steroid-resistant membranous nephropathy. (Grade B)

Meta-analysis of 18 RCTs including 1,025 cases published in 2004, confirmed that alkylating agents were more effective for the initial treatment of nephrotic membranous nephropathy than placebo or corticosteroid alone. Jha et al. showed that cyclophosphamide combined with corticosteroid significantly induced remission and suppressed the progression of renal dysfunction in membranous nephropathy.

In addition, a prospective study of 103 patients with nephrotic membranous nephropathy showed significant efficacy of treatment using cyclophosphamide combined with corticosteroid compared with a historical control.

In Japan, corticosteroid alone is recommended for the initial treatment of idiopathic membranous nephropathy in the Guidelines for the Treatment of Nephrotic Syndrome published in 2011 based on the data from a large cohort study of Japanese population. From the above data, cyclophosphamide combined with corticosteroid should be considered for the treatment of patients with steroid-resistant membranous nephropathy.

\section{Bibliography}

1. Perna A, et al. Am J Kidney Dis. 2004;44:385-401. (Level 1)

2. Ponticelli C, et al. J Am Soc Nephrol. 1998;9:444-50. (Level 2)

3. Jha V, et al. J Am Soc Nephrol. 2007;18:1899-904. (Level 2)

4. Hofstra JM, et al. Nephrol Dial Transplant. 2008;23:3534-8. (Level 4)

5. Naumovic R, et al. Biomed Pharmacother. 2010;64:633-8. (Level 4)

6. Shiiki H, et al. Kidney Int. 2004;65:1400-7. (Level 4)

7. Eriguchi M, et al. Nephrol Dial Transplant. 2009;24:3082-8. (Level 4)
CQ3 Is warfarin recommended for preventing thrombosis in patients with idiopathic membranous nephropathy?

We recommend warfarin for preventing thrombosis in patients with nephrotic membranous nephropathy. (Grade C1)

In nephrotic syndrome, a thromboembolic event is likely to occur because of an increased level of prothrombotic factors and decreased activity of the fibrinolytic system. In a large retrospective cohort study conducted in the US and Netherlands, a high incidence of thromboembolic events was reported in patients with nephrotic syndrome. Proteinuria and hypoalbuminemia were predictive factors for the development of venous thrombosis. Membranous nephropathy was the leading cause of renal vein thrombosis.

Markov model analysis using a hypothetical incidence of thromboembolic and hemorrhagic events suggested that preventive anticoagulation using warfarin decreased the incidence of thromboembolic events and prolonged life expectancy in patients with membranous nephropathy.

In nephrotic membranous nephropathy, the administration of warfarin therapy should be determined individually considering the patient's past history of thromboembolic events and degree of hypoalbuminemia.

\section{Bibliography}

1. Kayali F, et al. Am J Med. 2008;121:226-30. (Level 4)

2. Mahmoodi BK, et al. Circulation. 2008;117:224-30. (Level 4)

3. Cherng SC, et al. Clin Nucl Med. 2000;25:167-72. (Level 4)

4. Singhal R, et al. Thromb Res. 2006;118:397-407. (Level 4)

5. Bellomo R, et al. Nephron. 1993;63:240-1. (Level 4)

6. Sarasin FP, et al. Kidney Int. 1994;45:578-85. (Level 4)

CQ4 Are statins recommended for improving dyslipidemia in patients with idiopathic membranous nephropathy?

We recommend that statins be prescribed for dyslipidemia in patients with idiopathic membranous nephropathy. (Grade B)

Dyslipidemia in nephrotic syndrome is an important risk factor for the development of CVD, as well as for the progression of renal dysfunction. Several studies have reported on the efficacy and safety of statins for dyslipidemia in idiopathic membranous nephropathy. Association 
between statin use and a lower risk of venous thromboembolism or improvement of endothelial function has been reported. Because more than $50 \%$ of idiopathic membranous nephropathy cases in Japan develop at 65 years of age or older, their CVD risk is high. Therefore, the administration of statin is expected to prevent the development of CVD. The target values of LDL-cholesterol and non-HDLcholesterol should be less than 120 and $150 \mathrm{mg} / \mathrm{dl}$, respectively.

\section{Bibliography}

1. Rayner BL, et al. Clin Nephrol. 1996;46:219-24. (Level 3)

2. Fuiano G, et al. Nephron. 1996;73:430-5. (Level 3)

3. Valdivielso P, et al. Nephrology (Carlton). 2003;8:61-4. (Level 4)

4. Gazarin S, et al. J Nephrol. 2002;15:690-5. (Level 4)

5. Matzkies FK, et al. Am J Nephrol. 1999;19:492-4. (Level 4)

6. Olbricht CJ, et al. Kidney Int 1999;71 (Suppl):S113-6. (Level 2)

7. Brown CD, et al. Am J Kidney Dis. 1995;26:170-7. (Level 3)

8. Thomas ME, et al. Kidney Int. 1993;44:1124-9. (Level 2)

9. Shibasaki T, et al. Nihon Jinzo Gakkai Shi. 1993;35:1243-8. (Level 4)

10. Dogra GK, et al. Kidney Int. 2002;62:550-7. (Level 4)

11. Resh M, et al. Thromb Res. 2011;127:395-9. (Level 4)

CQ5 Are RAS inhibitors recommended for patients with idiopathic membranous nephropathy and hypertension?

We recommend RAS inhibitors to reduce proteinuria in patients with idiopathic membranous nephropathy and hypertension. (Grade B)

Hypertension often occurs as a complication of membranous nephropathy and is a risk factor for the progression of CKD. To treat such hypertension, restriction of sodium intake and administration of anti-hypertensive agents have been recommended. The anti-proteinuric effect of RAS inhibitors on diabetic and non-diabetic nephropathies is well known. Polanco et al. reported that treatment with RAS inhibitors was associated with a significantly increased probability of spontaneous remission of membranous nephropathy. RAS inhibitors are preferred as the first-line antihypertensive therapy and are expected to reduce urine protein and slow the progression of membranous nephropathy.

\section{Bibliography}

1. Polanco N, et al. J Am Soc Nephrol. 2010;21:697-704. (Level 4)

2. Kosmadakis G, et al. Scand J Urol Nephrol. 2010;44:251-6. (Level 2)

3. Iimura O, et al. Nihon Jinzo Gakkai Shi. 2003;45:439-44. (Level 4)
4. Prasher PK, et al. J Assoc Physicians India. 1999;47:180-2. (Level 4)

5. Ruggenenti P, et al. Am J Kidney Dis. 2000;35:381-91. (Level 4)

6. Praga M, et al. Nephrol Dial Transplant. 1997;12:2576-9. (Level 4)

7. Rostoker G, et al. Nephrol Dial Transplant. 1995;10:25-9. (Level 4)

8. Gansevoort RT, et al. Nephrol Dial Transplant. 1992;7(Suppl1):91-6. (Level 3)

9. Thomas DM, et al. Am J Kidney Dis. 1991;18:38-43. (Level 4)

10. Kincaid-Smith $P$, et al. Nephrol Dial Transplant. 2002;17:597-601. (Level 2)

CQ6 Is treatment with high-dose corticosteroid alone recommended for inducing remission in FSGS?

We recommend treatment with high-dose corticosteroid alone to patients with FSGS for initial induction of remission. (Grade B)

We recommend ciclosporin in combination with lowdose corticosteroid to patients with steroid-resistant FSGS. (Grade B)

An important prognostic indicator of FSGS is the initial response to therapy. Aggressive immunosuppressive therapy aimed at inducing remission is recommended because sustained nephrotic range proteinuria is a risk factor for progression to ESKD, and, conversely, responders to initial therapy have better long-term outcomes. There are no RCTs comparing corticosteroid or other agents to placebo as the first-line therapy for idiopathic FSGS. Observational studies have shown that high-dose corticosteroid could efficiently induce remission. Therefore, as the first-line therapy, steroid therapy aimed at inducing remission is recommended for patients with FSGS. In steroid-resistant patients, several studies have reported that treatment with ciclosporin in combination with low-dose corticosteroid is effective for inducing remission and preserving renal function.

\section{Bibliography}

1. Troyanov S, et al. J Am Soc Nephrol. 2005;16:1061-8. (Level 4)

2. Agarwal SK, et al. Nephron. 1993;63:168-71. (Level 4)

3. Frassinetti Castelo Branco Camurça Fernandes P, et al. J Nephrol. 2005;18:711-20. (Level 4)

4. Cattran DC, et al. Kidney Int. 1999;56:2220-6. (Level 2)

5. Lee HY, et al. Clin Nephrol. 1995;43:375-81. (Level 4)

6. Walker RG, et al. Nephron. 1990;54:117-21. (Level 2)

7. Ponticelli C, et al. Kidney Int. 1993;43:1377-84. (Level 2)

8. Braun N, et al. Cochrane Database Syst Rev. 2008;3:CD00 3233. (Level 1)

9. Senthil Nayagam L, et al. Nephrol Dial Transplant. 2008;23:1926-30. (Level 2) 
10. Westhoff TH, et al. Clin Nephrol. 2006;65:393-400. (Level 4)

11. Cattran DC, et al. Clin Nephrol. 2004;62:405-11. (Level 4)

12. Martinelli R, et al. Braz J Med Biol Res. 2004;37:1365-72. (Level 3)

13. Heering P, et al. Am J Kidney Dis. 2004;43:10-8. (Level 2)

CQ7 Is LDL apheresis recommended for reducing urinary protein levels in patients with FSGS?

In patients with steroid-resistant FSGS with a high LDL cholesterol level, we suggest the use of LDL apheresis therapy for reducing urinary protein. (Grade C1)

LDL apheresis is expected not only to improve dyslipidemia, but also to reduce proteinuria and preserve renal function via immunomodulation in refractory nephrotic syndrome.

Several nonrandomized studies using variable schedules of LDL apheresis in patients with steroid-resistant FSGS have demonstrated some benefits in terms of reducing proteinuria and improving the serum albumin concentration. The health insurance system in Japan supports the use of LDL apheresis 12 times within 3 months for refractory nephrotic syndrome with a high LDL cholesterol level.

\section{Bibliography}

1. Tojo K, et al. Jpn J Nephrol. 1988;30:1153-60. (Level 5)

2. Muso E, et al. Nephron. 2001;89:408-15. (Level 4)

3. Hattori M, et al. Am J Kidney Dis. 2003;42:1121-30. (Level 5)

4. Muso E, et al. Clin Nephrol. 2007;67:341-4. (Level 4)

\section{Chapter 12: Autosomal-dominant polycystic kidney disease (ADPKD)}

CQ1 Is anti-hypertensive treatment recommended as a means of slowing the deterioration of renal function in hypertensive patients with ADPKD?

We recommend antihypertensive treatment for hypertensive patients with ADPKD, because it may slow the deterioration of renal function. (Grade $\mathrm{C} 1$ )

Hypertension in ADPKD is frequent and develops from youth in contrast to essential hypertension. In addition, it is often recognized when the renal function is normal and the cysts are still small. Anti-hypertensive treatment is generally performed. Although the evidence related to recommended anti-hypertensive agents and the target blood pressure is inconclusive, antihypertensive treatment is thought to slow the deterioration of renal function in hypertensive patients with ADPKD.

\section{Bibliography}

1. Cadnapaphornchai MA, et al. Clin J Am Soc Nephrol. 2009;4:820-9. (Level 2)

2. Sarnak MJ, et al. Ann Intern Med. 2005;142:342-51. (Level 2)

3. Schrier RW, et al. Kidney Int. 2003;63:678-85. (Level 4)

4. Jafar TH, et al. Kidney Int. 2005;67:265-71. (Level 1)

5. Maschio G, et al. N Engl J Med. 1996;334:939-45. (Level 2)

6. van Dijk MA, et al. Nephrol Dial Transplant. 2003;18:2314-20. (Level 2)

7. Ecder T, et al. Am J Kidney Dis. 2000;35:427-32. (Level 2)

8. Schrier R, et al. J Am Soc Nephrol. 2002;13:1733-9. (Level 2)

9. Kanno Y, et al. QJM. 1996;89:65-70. (Level 4)

10. Nutahara K, et al. Nephron Clin Pract. 2005;99:c18-23. (Level 2)

11. Mitobe M, et al. Clin Exp Nephrol. 2010;14:573-7. (Level 4)

12. Zeltner R, et al. Nephrol Dial Transplant. 2008;23:573-9. (Level 2)

13. Ecder T, et al. Am J Nephrol. 2001;21:98-103. (Level 3)

CQ2 Does screening of intracranial aneurysms improve the prognosis in patients with ADPKD?

The prevalence of unruptured intracranial aneurysms in ADPKD is higher than in the general population. Intracranial hemorrhage, either cerebral hemorrhage or aneurysmal subarachnoid hemorrhage, can cause high mortality and morbidity in PKD patients. Screening of intracranial aneurysms improves the prognosis.

The high incidence of intracranial aneurysms in patients with ADPKD has long been recognized. Rupture of an intracranial aneurysm resulting in subarachnoid hemorrhage (SAH) is the most devastating of extrarenal complications, and often results in premature death or disability. The overall prevalence was estimated to be $3.2 \%$ (95\% CI 1.9-5.2) in a population without comorbidity, with a mean age of 50 years, and a $50 \%$ component of men. Compared with populations without comorbidity, the prevalence ratio was 6.9 (95\% CI 3.5-14) for ADPKD. First-degree relatives (parents, siblings, and children) of patients with subarachnoid hemorrhage have a three to seven times higher risk of SAH than the general population.

Size of the aneurysm size correlates with the presence of symptoms and the risk of bleeding, and aneurysms may rupture more often and at a younger age as compared with sporadic aneurysms. Large aneurysms seem to occur more often in patients with ADPKD than in those without. Intracranial hemorrhage, either cerebral hemorrhage or aneurysmal SAH, can cause high mortality and 
morbidity in PKD patients. Screening of intracranial aneurysms improves the prognosis.

If the result of screening by MR angiography is negative, rescreening of patients with a good life expectancy at 5-year intervals seems reasonable.

\section{Bibliography}

1. Chauveau D, et al. Kidney Int. 1994;45:1140-6. (Level 4)

2. Schievink WI, et al. J Am Soc Nephrol. 1992;3:88-95. (Level 4)

3. Vlak MH, et al. Lancet Neurol. 2011;10:626-36. (Level 4)

4. Irazabal MV, et al. Clin J Am Soc Nephrol. 2011;6:1274-85. (Level 4)

5. Xu HW, et al. Stroke. 2011;42:204-6. (Level 4)

6. Gieteling EW, et al. J Neurol. 2006;250:418-23. (Level 4)

7. Wiebers DO, et al. Lancet. 2003;362:103-10. (Level 4)

CQ3 Are newer quinolones recommended for the treatment of cyst infection in ADPKD?

We suggest administration of the newer quinolones for the treatment of cyst infection in ADPKD. (Grade C1)

Cyst infection is a frequent and serious complication of ADPKD and is often refractory and difficult to treat. Most causative bacteria originate from the intestine and many are gram-negative rods. Fluoroquinolones, which have broad effectiveness against gram-negative rods and good penetration into cysts, is recommended for the treatment of infected cysts in ADPKD. Having said this, however, there has not been an adequate level of study to investigate the actual effectiveness of fluoroquinolones for treating cyst infection in ADPKD. There have been few studies comparing fluoroquinolones with other antibiotics for the treatment of cyst infection in ADPKD.

\section{Bibliography}

1. Alam A, et al. Clin J Am Soc Nephrol. 2009;4:1154-5. (Level 6)

2. Sallée M, et al. Clin J Am Soc Nephrol. 2009;4:1183-9. (Level 5)

3. Suwabe T, et al. Nephron Clin Pract. 2009;112:C157-63. (Level 5)

4. Schwab SJ, et al. Am J Med. 1987;82:714-18. (Level 5)

5. Muther RS, et al. Kidney Int. 1981;20:519-22. (Level 5)

6. Bennet WM, et al. Am J Kid Dis. 1985;6:400-4. (Level 5)

7. Schwab SJ, et al. Am J Kid Dis. 1983;3:63-6. (Level 5)

8. Elzinga LW, et al. Kidey Int. 1987;32:884-8. (Level 5)

9. Elzinga LW, et al. Antimicrob Agents Chemother. 1988;32:844-7. (Level 5)

10. Telenti A, et al. Mayo Clin Proc. 1990;65:933-42. (Level 5)
11. Rossi SJ, et al. Ann Pharmacother. 1993;27:38-9. (Level 5)

12. Hiyama L, et al. Am J Kidney Dis. 2006;47:E9-13. (Level 5)

CQ4 Do renal volume and the speed of its enlargement reflect the prognosis of renal function?

Renal volume and the speed of its enlargement reflect the prognosis of renal function.

In patients with ADPKD, renal cysts grow exponentially. It has been reported that the median change in eGFR per year was almost $2-5 \mathrm{~mL} / \mathrm{min} / 1.73 \mathrm{~m}^{2}$. Since the remaining renal parenchyma has the capacity to compensate for the loss of GFR, the GFR may be sustained until the disease progresses. Although GFR is the usual biomarker of renal disease progression, it does not decrease substantially until extensive and irreversible damage to noncystic parenchyma occurs. Therefore, it is necessary to identify some reliable biomarkers to follow the progression of this disease. Recent data from the American study indicate that kidney growth is a critical predictor of progression to renal failure in Caucasian patients with $\mathrm{AD}$ $\mathrm{PKD}$, playing a more important role than hypertension, proteinuria, age, or sex. It was reported that total kidney volume (TKV) increased at a mean rate in the range from 4.0 to $9.4 \%$, almost $20-50 \mathrm{~cm}^{3}$ per year in several studies. Consequently, TKV growth is considered the best surrogate marker predicting the decline of renal function in ADPKD. Therefore, since there is no general agreement on the frequency of imaging evaluation, it is reasonable to follow up every 2-5 years in patients with a TKV of $1,000 \mathrm{ml}$ or less and every 1-2 years in patients with a larger TKV.

\section{Bibliography}

1. Grantham JJ, et al. N Engl J Med. 2006;354:2122-30. (Level 4)

2. Fick-Brosnahan GM, et al. Am J Kidney Dis. 2002;39:1127-34. (Level 4)

3. Tokiwa S, et al. Clin Exp Nephrol. 2011;15:539-45. (Level 4)

4. Cadnapaphornchai MA, et al. Clin J Am Soc Nephrol. 2011;6:369-76. (Level 4)

5. Cadnapaphornchai MA, et al. Kidney Int. 2008;74:1192-6. (Level 4)

6. Helal I, et al. Clin J Am Soc Nephrol. 2011;6:2439-43. (Level 4)

7. Chapman AB, et al. Kidney Int. 2003;64:1035-45. (Level 4)

8. Kistler AD, et al. Kidney Int. 2009;75:235-41. (Level 4)

9. Grantham JJ, et al. Clin J Am Soc Nephrol. 2010;5:889-96. (Level 4)

10. Meijer E, et al. Clin J Am Soc Nephrol. 2010;5:1091-8. (Level 4)

11. Seeman T, et al. Blood Press Monit. 2003;8:107-10. (Level 4) 


\section{Chapter 13: Rapidly progressive glomerulonephritic syndrome}

\section{RPGN and CKD}

RPGN(rapidly progressive glomerulonephritis)is defined by the World Health Organization (WHO) as "a syndrome of diseases presenting with insidiously developing hematuria and proteinuria and rapidly progressive renal failure," and in Japan as "a syndrome of diseases in which renal failure subacutely develops for several weeks to months associated with urine abnormalities indicative of glomerulonephritis" (Table 8). RPGN includes a wide variety of rapidly progressive renal diseases (ANCA-positive RPGN, lupus nephritis, anti-GBM antibody glomerulonephritis, etc.) and the definition does not require reference to the renal pathology, which often shows necrotizing and crescentic glomerulonephritis. The prognosis is poor as the initial therapy is delayed, thus it is important to make a diagnosis as early as possible according to the "diagnostic criteria for the early detection of RPGN" (Tables 8, 9).

Regarding the relationship between RPGN and CKD, of note is that differentiating RPGN from CKD (chronic

Table 8 Diagnostic criteria for rapidly progressive glomerulonephritis

(1) Progressively declining renal function during the course of several weeks to months (Information on renal function should be obtained from the present illness, previous checkup and other laboratory data)

(2) Nephritic urine including hematuria (mostly occult hematuria, rarely gross hematuria), proteinuria and casts

(3) Differentiation from chronic kidney disease using an imaging examination, such as renal ultrasonography and CT scanning based on whether or not renal/cortex atrophy and hydronephrosis are present, if previous data or the results of urinalysis are not immediately available

Table 9 Diagnostic criteria for early detection of rapidly progressive glomerulonephritis

(1) Urine abnormalities (esp. hematuria, proteinuria, casts)

(2) eGFR $<60 \mathrm{~mL} / \mathrm{min} / 1.73 \mathrm{~m}^{2}$

(3) Elevated CRP and ESR

* If the above criteria are fulfilled, referral to a nephrology clinic is recommended after confirming the absence of renal cortex atrophy by ultrasonography, if available. If infection or exacerbation of chronic nephritis is suspected, serum creatinine should be reexamined and the eGFR value calculated after 1 or 2 weeks

There is an increasing number of cases of RPGN that initially only show asymptomatic urine findings. With the occurrence of a recently appearing urine abnormality, RPGN should be considered even if the renal function appears to be almost normal

eGFR should be calculated by the equation used for the Japanese glomerulonephritic syndrome) is not possible with only one visit. Therefore, the possibility of RPGN should be considered even if the patient's serum creatinine level remains slightly above or even within the reference values, because serum creatinine does not necessarily reflect renal function within that low range of values. Thus, it is important to reexamine the renal function within several weeks.

Some of the patients with RPGN will be followed as CKD after their initial therapy. Such patients may be managed according to the clinical practice guidelines for CKD in addition to maintenance immunosuppressive therapy. RPGN may develop de novo, or as an exacerbation of chronic glomerulonephritis during the course of CKD. Small kidney size generally suggests the presence of CKD, but the fact that RPGN can develop from CKD cannot be ignored.

CQ-1 Are corticosteroids recommended as initial therapy for RPGN?

We recommend oral or intravenous corticosteroids at higher than moderate doses for the initial therapy of patients with ANCA-positive RPGN. (Grade A) In patients with severe ANCA-positive RPGN, we recommend adding intravenous pulse corticosteroid therapy to oral corticosteroids to achieve rapid, strong anti-inflammatory and immunosuppressive effects. (Grade B)

In patients with anti-GBM antibody disease presenting with RPGN, we recommend high doses of corticosteroids including intravenous pulse therapy, combined with immunosuppressive agents and plasmapheresis to improve kidney and patient survival. (Grade B)

Corticosteroids are widely used as initial therapy for various causes of RPGN. This chapter presents recommendations for the treatment of two typical diseases, ANCA-positive RPGN and anti-GBM antibody disease.

Initial therapy including corticosteroids for patients with ANCA-positive RPGN is considered for the following cases:

Cases in which a good response to aggressive treatment can be expected, considering the acute-onset time course with a predominance of active inflammation findings based on renal biopsy if available.

Cases showing acute inflammation and systemic vasculitic symptoms that cannot be explained by infection Cases not complicated by infection or those whose infection is appropriately controlled by antimicrobial agents or other preventive measures. 
Cases in whom the benefits will outweigh the risks of using corticosteroids, considering the patient's age and the presence or absence of osteopenia, etc.

In patients with anti-GBM antibody glomerulonephritis presenting with RPGN, corticosteroids, in combination with immunosuppressive agents and/or plasmapheresis, are recommended if the patient is complicated by pulmonary hemorrhage, or a response to the therapy is expected if the patient is not dialysis-dependent, and does not show a severe lesion in the majority of the glomeruli in the renal biopsy if available.

\section{Bibliography}

1. Frohnert PP, et al. Am J Med. 1967;43:8-14. (Level 5)

2. Bolton WK, et al. Am J Med. 1979;66:495-502. (Level 5)

3. Couser WG. Am J Nephrol. 1982;2:57-69. (Level 5)

4. Nachman PH, et al. J Am Soc Nephrol. 1996;7:33-9. (Level 3)

5. Hogan SL, et al. Ann Intern Med. 2005;143:621-31. (Level 4)

6. Adu D, et al. QJM. 1997;90:401-9. (Level 2)

7. Bolton WK, et al. Am J Nephrol. 1989;9:368-75. (Level 4)

8. Hogan SL, et al. J Am Soc Nephrol. 1996;7:23-32. (Level 4)

9. de Lind van Wijngaarden RA, et al. J Am Soc Nephrol. 2006;17:2264-74. (Level 4)

10. Levy JB, et al. Ann Intern Med. 2001;134:1033-42. (Level 4)

11. Johnson JP, et al. Medicine (Baltimore). 1985;64:219-27. (Level 2)

CQ2 Are immunosuppressants recommended for patients with RPGN?

We recommend immunosuppressants for use as the initial treatment and maintenance therapy of patients with ANCA-positive RPGN. (Grade A)

In patients with Anti-GBM antibody-positive RPGN in whom the effects of corticosteroid are inadequate or whose dose of corticosteroid cannot be reduced, we recommend immunosuppressants for use as the initial treatment and maintenance therapy. (Grade B)

ANCA-associated vasculitis (AAV) is a frequent and clinically important disease in RPGN. Prospective studies in patients with AAV have been performed in European countries and the US. On the other hand, anti-GBM antibody-positive RPGN is rare disease and kidney survival is poor. Retrospective studies in patients with anti-GBM antibody-positive RPGN have been performed in European countries, the US and China.

1. Initial treatment of ANCA-positive RPGN

Treatment with corticosteroids and cyclophosphamide has improved the outcome of patients with ANCA-positive RPGN. We recommend daily oral cyclophosphamide (25-100 mg per day) or intravenous pulses of cyclophosphamide (250-750 mg/m $\mathrm{m}^{2}$ per month) with corticosteroids at the initial treatment, considering the clinical grade, patient's age and requirement for dialysis.

2. Initial treatment of anti-GBM antibody-positive RPGN The clinical guidelines in Japan recommend immunosuppressive therapy (corticosteroids and cyclophosphamide) plus plasmapheresis. We recommend cyclophosphamide (1-2 $\mathrm{mg} / \mathrm{kg}$ per day) for patients with refractory GN. However, it is necessary to reduce the dose of cyclophosphamide for patients with advanced renal dysfunction.

3. Maintenance therapy of ANCA-positive RPGN Cyclophosphamide along with azathioprine, mizoribine, mycophenolate mofetil and methotrexate have been reported as immunosuppressants in patients with AAV. Treatment with azathioprine or mizoribine in patients with ANCA-positive RPGN is recommended as maintenance therapy to prevent a relapse.

\section{Bibliography}

1. Koyama A, et al. Clin Exp Nephrol. 2009;13:633-50. (Level 4)

2. Ozaki S, et al. Mod Rheumatol. 2012;22(3):394-404. (Level 4)

3. Jayne D, et al. N Engl J Med. 2003;349:36-44. (Level 2)

4. Hirayama K, et al. Am J Kidney Dis. 2004;44:57-63. (Level 5)

5. Hiemstra TF, et al. JAMA. 2010;304:2381-8. (Level 2)

6. Langford CA, et al. Arthritis Rheum. 1999;42:2666-73. (Level 3)

7. Langford CA, et al. Am J Med. 2003;114:463-9. (Level 4)

CQ3 Is the addition of plasmapheresis to treatment recommended in patients with RPGN?

We recommend the addition of plasmapheresis to treatment for patients with ANCA-positive RPGN presenting either with advanced kidney failure or with diffuse alveolar hemorrhage. (Grade $\mathrm{C} 1$ )

We recommend the addition of plasmapheresis to treatment for patients with anti-GBM antibody-positive RPGN. (Grade B)

Treatment with immunosuppressive therapy plus plasmapheresis has improved the outcome of patients with RPGN. Prospective studies in patients with ANCA-associated vasculitis (AAV) and retrospective studies in patients with anti-GBM antibody-positive RPGN have been performed in European countries and the US.

1. ANCA-positive RPGN

ANCA is thought to be involved in the clinical conditions of AAV and RPGN. The removal of ANCA may, therefore, result in controlling disease activity and preventing organ damage. Addition of plasmapheresis to the initial therapy with corticosteroids and 
cyclophosphamide is indicated for patients presenting with either advanced kidney failure $(\mathrm{sCr}>5.8 \mathrm{mg} / \mathrm{dl})$ or with diffuse alveolar hemorrhage.

2. Anti-GBM antibody-positive RPGN

We recommend plasmapheresis to improve the outcome of patients with anti-GBM antibody-positive RPGN. On the other hand, in patients with advanced kidney failure or dialysis, there is only rare evidence that plasmapheresis improves the outcome.

3. Immune complex RPGN

We recommend plasmapheresis for patients with immune complex RPGN, while considering the patient's age, organ damage and pathological findings.

\section{Bibliography}

1. Jayne DR, et al. J Am Soc Nephrol. 2007;18:2180-8. (Level 2)

2. Szpirt WM, et al. Nephrol Dial Transplant. 2011;26:206-13. (Level 2)

3. Walters GD, et al. BMC Nephrol. 2010;11:12. (Level 1)

4. Walsh M, et al. Am J Kidney Dis. 2011;57:566-74. (Level 1)

5. Yamagata K, et al. J Clin Apher. 2005;20:244-51. (Level 4)

6. Cui Z, et al. Medicine (Baltimore). 2011;90:303-11. (Level 4)

7. Flores JC, et al. Lancet 1986;1:5-8. (Level 5)

CQ4 Are corticosteroids recommended

for maintenance therapy in patients with RPGN?

We recommend the use of corticosteroids for maintenance therapy in patients with RPGN. (Grade B)

After remission due to the initial treatment, maintenance therapy is needed to prevent a relapse. Low doses of oral corticosteroid with immunosuppressant are used as maintenance therapy mainly in European countries and the US.

\section{ANCA-positive RPGN}

We recommend a corticosteroid dose of less than $10 \mathrm{mg} /$ day orally as maintenance therapy and suggest continuing administration for 12-18 months in patients who remain in complete remission. A study reported that a reduction rate above $0.8 \mathrm{mg} / \mathrm{month}$ was associated with a higher relapse rate. Shortening the treatment period should be considered in aged or dialysis-dependent patients.

2. Anti-GBM antibody-positive RPGN

There is rare evidence in patients with anti-GBM antibody-positive RPGN. We suggest continuing corticosteroid for more than 6-9 months as maintenance therapy.

\section{Bibliography}

1. Jayne D, et al. N Engl J Med. 2003;349:36-44. (Level 2)

2. De Groot K, et al. Arthritis Rheum. 2005;52:2461-9. (Level 2)

3. Walsh M, et al. Arthritis Care Res. 2010;62:1166-73. (Level 4)
4. Wada T, et al. J Rheumatol. 2012;39:545-51. (Level 4)

5. Ozaki S, et al. Mod Rheumatol. 2012;22:394-404. (Level 4)

6. Levy JB, et al. Ann Intern Med. 2001;134:1033-42. (Level 4)

\section{Chapter 14: Dyslipidemia in CKD}

CQ1 What lipid-lowering medications are safe and recommended for CKD?

We recommend statin alone or the statin-ezetimibe combination as safe lipid-lowering medications for CKD. (Grade A)

To avoid adverse effects, we recommend avoiding the use of fibrates, which are excreted mainly through the kidneys in subjects with CKD G4 or more advanced stages. (Grade D)

Fibrates are often chosen for the treatment of hypertriglyceridemia in the general population. However, the use of major fibrates, such as bezafibrate and fenofibrate, are contraindicated in patients with severe renal impairment. According to the package inserts, bezafibrate and fenofibrate should not be given to subjects with an increased serum creatinine level of $2.0 \mathrm{mg} / \mathrm{dL}$ or higher, and in those with a serum creatinine level of $2.5 \mathrm{mg} / \mathrm{dL}$ or higher, respectively. To avoid adverse effects, we do not recommend the use of fibrates, which are excreted mainly through the kidney in subjects with CKD G4 or more advanced stages.

Regarding the use of statin in CKD patients, although rhabdomyolysis and other adverse effects may be of concern, previous individual studies and meta-analyses showed that statins, as compared with placebo, were safe to use in patients with CKD including dialysis patients. A combination of statin and ezetimibe was also found to be safe as shown in the SHARP trial.

Care should be taken when a statin is co-administered with other drugs. Statin in combination with a fibrate is contraindicated in subjects with renal impairment. Cyclosporin increases the serum concentration of a statin by inhibiting OATP1B1. Statins metabolized by CYP3A4 can be accumulated when administered with grapefruit juice, itraconazol and other drugs inhibiting CYP3A4.

Colestimide, probucol and eicosapentaenoic acid ethyl icosapentate may be used at the same dosage as with nonCKD patients. The dose of niceritrol should be reduced according to the patient's kidney function. There is no evidence, however, that these lipid-lowering drugs reduce the CVD risk in patients with CKD.

\section{Bibliography}

1. Nakamura H, et al. Atherosclerosis. 2009;206:512-7. (Level 4)

2. Strippoli GF, et al. BMJ. 2008;336:645-51. (Level 1)

3. Baigent C, et al. Lancet. 2011;377:2181-92. (Level 2) 
CQ2 Is lipid-lowering treatment recommended to prevent CVD events in CKD?

We recommend lipid-lowering treatment for CKD patients to prevent CVD. (Grade B)

We recommend that the target LDL-C level be $<120 \mathrm{mg} / \mathrm{dl}$ and the target non-HDL-C level be $<150 \mathrm{mg} / \mathrm{dl}$ in primary prevention of coronary artery disease, and that the target LDL-C level be $<100 \mathrm{mg} / \mathrm{dl}$ and the target non-HDL-C level be $<130 \mathrm{mg} / \mathrm{dl}$ in secondary prevention, in CKD. (Grade $\mathrm{C} 1$ )

CKD patients constitute a high-risk population for CVD. Dyslipidemia is one of the established risk factors for atherosclerotic CVD. CKD patients show various phenotypes of dyslipidemia, such as type IIa, IIb, and IV in nephrotic syndrome, and type III and IV in renal failure. There is only a limited amount of information about whether dyslipidemia contributes to an increased CVD risk in CKD. In the ARIC study in the US, higher levels of serum total cholesterol and triglycerides were predictive of a higher risk of ischemic heart disease regardless of the baseline eGFR. In a large cohort of Japanese hemodialysis patients, both higher non-HDL-cholesterol and lower HDL-cholesterol were independent predictors of incident myocardial infarction. These results support the notion that dyslipidemia is a risk factor of atherosclerotic CVD in CKD as well as in non-CKD populations.

Randomized controlled trials (RCTs) in CKD have shown mixed results. Statins failed to decrease the risk of primary cardiovascular endpoints in hemodialysis patients (4D and AURORA). The SHARP trial showed a significant $17 \%$ reduction in CVD risk by the administration of $20 \mathrm{mg}$ simvastatin in combination with $10 \mathrm{mg}$ ezetimibe in subjects with CKD categories G3 to G5D. In the subgroup analysis of SHARP, predialysis patients at baseline showed a significant $20 \%$ reduction of CVD risk, whereas those on dialysis at baseline showed an insignificant risk reduction by $10 \%$. Analyses of SHARP and 4D, stratified by baseline lipid levels, indicated that patients with higher baseline total or LDL-cholesterol levels benefited more than those with lower levels. In addition, sub-analyses of CKD stage G3 derived from previous RCTs using statins revealed a larger reduction of relative risk than the original total cohort. We interpreted these data to indicate that lipid-lowering treatment is effective in reducing atherosclerotic CVD in CKD, but that the benefit of such treatment varies at different stages of CKD and at different baseline lipid levels.

We recommend that the target LDL-C and non-HDL-C levels be $<120$ and $<150 \mathrm{mg} / \mathrm{dL}$, respectively for primary prevention, and $<100$ and $<130 \mathrm{mg} / \mathrm{dL}$, respectively for secondary prevention. These target levels are in accordance with the recommendations for CKD in the Japan Atherosclerosis Society Guidelines for the Diagnosis and Prevention of Atherosclerotic Cardiovascular Disease in Japan-2012 Version.

\section{Bibliography}

1. Ninomiya T, et al. Kidney Int. 2005;68:228-36. (Level 4)

2. Ninomiya T, et al. Circulation. 2008;118:2694-701. (Level 4)

3. Irie F, et al. Kidney Int. 2006;69:1264-71. (Level 4)

4. Kokubo Y, et al. Stroke. 2009;40:2674-9. (Level 4)

5. Muntner P, et al. J Am Soc Nephrol. 2005;16:529-38. (Level 4)

6. Shoji T, et al. Clin J Am Soc Nephrol. 2011;6:1112-20. (Level 4)

7. Wanner C, et al. N Engl J Med. 2005;353:238-48. (Level 2)

8. Fellström BC, et al. N Engl J Med. 2009;360:1395-407. (Level 2)

9. Baigent C, et al. Lancet. 2011;25:2181-92. (Level 2)

10. Shepherd J, et al. J Am Coll Cardiol. 2008;51:1448-54. (Level 4)

11. Koren MJ, et al. Am J Kidney Dis. 2009;53:741-50. (Level 4)

12. Nakamura H, et al. Atherosclerosis. 2009;206:512-7. (Level 4)

13. Vidt DG, et al. Clin Ther. 2011;33:717-25. (Level 4)

14. Tonelli M, et al. Circulation. 2005;112:171-8. (Level 4)

15. Shimano H, et al. J Atheroscler Thromb. 2008;15:116-21. (Level 4)

16. Okamura T, et al. Atherosclerosis. 2009;203:587-92. (Level 4)

CQ3 Is statin therapy recommended for CKD patients to suppress the progression of CKD?

We recommend Statin therapy to reduce proteinuria in CKD. (Grade B)

We recommend Statin therapy to suppress kidney function loss in CKD. (Grade C1)

Treatment of dyslipidemia has been established for both primary and secondary prevention of atherosclerotic cardiovascular events. There are studies showing the effects of lipid-lowering treatment on proteinuria and kidney function in CKD.

Observational studies in the general population and type 1 diabetic patients showed that dyslipidemia was a predictor for the development of albuminuria, proteinuria, and CKD. One study showed the effect of statin on proteinuria in users of renin-angiotensin-system inhibitors. Other studies suggested dose-dependency of statin effects on proteinuria and eGFR. The effect of lipid-lowering with a statin on proteinuria in CKD patients was the subject of three meta-analyses, and all supported the anti-proteinuric effect of statin. In addition to statins, there have been studies reporting the anti-proteinuric effects of fibrates, and ezetimibe in combination with a statin. LDL-apheresis is known to suppress proteinuria and is indicated for refractory nephrotic syndrome in Japan. 
Regarding the effect of lipid-lowering treatment with a statin on kidney function, three meta-analyses have been performed with inconsistent results; one yielded positive and two yielded neutral results on eGFR. These metaanalyses were different in the number and background of the study subjects. Original individual studies have reported mixed results. These variable results may be due to differences in the study design, sample size, co-morbidities and CKD stages of the subjects, and medications tested. In the SHARP trial, treatment with ezetimibe-statin combination was not effective in preserving kidney function.

Although the precise mechanisms by which statins exert reno-protection are unknown, such actions may be mediated by their reduction and improvement of the serum lipid profile and their pleiotropic actions such as anti-inflammation, protection of renal tubular damage, suppression of AGE production, and their anti-oxidative properties.

\section{Bibliography}

1. Whaley-Connell A, et al. J Clin Hypertens (Greenwich). 2010;12:51-8. (Level 4)

2. O'Seaghdha CM, et al. Am J Kidney Dis. 2010;56:852-60. (Level 4)

3. Raile K, et al. Diabetes Care. 2007. 30:2523-8. (Level 4)

4. Sandhu S, et al. J Am Soc Nephrol. 2006;17:2006-16. (Level 1)

5. Navaneethan SD, et al. Cochrane Database Syst Rev. 2009;15:CD007784. (Level 1)

6. Strippoli GF, et al. BMJ. 2008;336:645-51. (Level 1)

7. Bianchi S, et al. Am J Kidney Dis. 2003;41:565-70. (Level 2)

8. Bianchi S, et al. Am J Kidney Dis. 2010;55:671-81. (Level 2)

9. Shepherd J, et al. Clin J Am Soc Nephrol. 2007;2:1131-9. (Level 4)

10. Keech A, et al. Lancet. 2005;366:1849-61. (Level 2)

11. Landray M, et al. Am J Kidney Dis. 2006;47:385-95. (Level 2)

12. Baigent C, et al. Lancet. 2011;377:2181-92. (Level 2)

13. Kimura K, et al. J Atheroscler Thromb. 2010;17:601-9. (Level 4)

14. Colhoun HM, et al. Am J Kidney Dis. 2009;54:810-9. (Level 4)

15. Fassett RG, et al. Atherosclerosis. 2010;213:218-24. (Level 4)

16. Tonelli M, et al. Circulation. 2005;112:171-8. (Level 4)

17. Vidt DG, et al. Clin Ther. 2011;33:717-25. (Level 4)

18. Ruggenenti P, et al. Clin J Am Soc Nephrol. 2010;5:1928-38. (Level 2)

19. Rahman M, et al. Am J Kidney Dis. 2008;52:412-24. (Level 4)

20. Huskey J, et al. Atherosclerosis. 2009;205:202-6. (Level 4)

21. Lemos PA, et al. Am J Cardiol. 2005;95:445-51. (Level 4)

22. Renke M, et al. Acta Biochim Pol. 2010;57:547-2. (Level 2)

23. Nakamura T, et al. Oxid Med Cell Longev. 2010;3:304-7. (Level 4)

24. Inoue T, et al. Intern Med. 2011;50:1273-8. (Level 4)

\section{Chapter 15: Obesity and Metabolic Syndrome in CKD}

CQ1 Is the metabolic syndrome a risk factor for the development of CKD?

The metabolic syndrome is a risk factor for the development of albuminuria and renal failure, except in premenopausal women and in CKD stage G4 and G5 subjects.

Improvement of the metabolic syndrome was accompanied by a reduction of albuminuria and stable GFR in subjects with type 2 diabetes mellitus.

The metabolic syndrome (MetS) is a cluster of risk factors for cardiovascular diseases, and could affect kidneys through various pathways. This section summarizes the epidemiological data showing MetS as a risk factor for the development of CKD. The association of MetS with CDK varies with gender, race, and age, which should be considered in the interpretation of the studies.

A recent meta-analysis has shown a significant association between MetS and the development of eGFR $<60 \mathrm{ml} /$ min per $1.73 \mathrm{~m}^{2}$. Each of the five components of MetS showed a positive association with this risk, and the strength of association increased as the number of components increased. MetS was also associated with the development of albuminuria. In the MAGIC study, it was concluded that concomitant occurrence of MetS and albuminuria increased the risk of kidney function loss more than five-fold compared to subjects with neither of these factors. Histologically, kidneys from MetS subjects showed a greater prevalence of tubular atrophy, interstitial fibrosis, arterial sclerosis, and global and segmental glomerulosclerosis than non-MetS subjects. MetS was also associated with renal dysfunction after kidney transplantation. In MetS subjects, non-alcoholic fatty liver disease (NAFLD), and especially liver fibrosis in non-alcoholic steatohepatitis (NASH) were associated with a decrease in kidney function. Change in body weight is better than body weight itself as a predictor of renal outcome. A retrospective cohort study showed that improvement of MetS was accompanied by reduced albuminuria and stable GFR in type 2 diabetes mellitus. An increased fat mass promoting inflammation is thought to be the major cause of obesityrelated nephropathy. 
Exceptions were that MetS was not a predictor of renal failure in CKD stage G4 and G5 subjects. Moreover, MetS was not associated with CKD in premenopausal women. These facts indicate the significant roles of age, sex, and CKD stages in the prediction of renal outcomes in MetS.

\section{Bibliography}

1. Thomas G, et al. Clin J Am Soc Nephrol. 2011;6:2364-73. (Level 4)

2. Leoncini G, et al. J Hum Hypertens. 2012;26:149-56. (Level 4)

3. Alexander MP, et al. Am J Kidney Dis. 2009;53:751-9. (Level 4)

4. Ozdemir FN, et al. Transplant Proc. 2010;41:2808-10. (Level 4)

5. Bello AK, et al. Nephrol Dial Transplant. 2007;22:1619-27. (Level 4)

6. Targher G, et al. Clin J Am Soc Nephrol. 2010;5:2166-71. (Level 4)

7. Arase Y, et al. Intern Med. 2011;50:1081-87. (Level 4)

8. Ryu S, et al. J Am Soc Nephrol. 2008;19:1798-805. (Level 4)

9. Axelsson J, et al. Am J Kidney Dis. 2006;48:916-25. (Level 4)

10. Mirza MA, et al. Arterioscler Thromb Vasc Biol. 2011;31:219-27. (Level 4)

11. Lee CC, et al. Clin Nephrol. 2011;75:141-9. (Level 4)

12. $\mathrm{Yu} \mathrm{M}$, et al. Nephrol Dial Transplant. 2010;25:469-77. (Level 4)

13. Duran-Perez EG, et al. Metab Syndr Relat Disord. 2011;9:483-9. (Level 4)

CQ2 Is intervention for the metabolic syndrome recommended to prevent the development of CKD?

Because of its anti-albuminuric and potentially renoprotective actions, we recommend a reduction in body weight and visceral fat mass by restricting energy intake in subjects with CKD and MetS, with the exception of premenopausal women and CKD stage G4 and G5. (Grade C1)

The kidney damage in MetS originates from multiple sources, including inflammation, high blood pressure, dyslipidemia, and impaired glucose tolerance. Accumulation of visceral fat in MetS plays a central role in these abnormalities. Therefore, weight loss, exercise, and a diet low in energy and fat have been used as first line interventions for MetS. Weight reduction achieved by lifestyle intervention reduces blood pressure and albuminuria, but there are no consistent results for renal function. This may be partly explained by the short intervention periods. Since obesity and MetS promote glomerular hyperfiltration, weight reduction would normalize the filtration load and reduce albuminuria. This GFR reduction (normalization) in the short-term does not necessarily indicate deterioration of renal function in the long-term.
Lifestyle intervention was shown to reduce body weight by $8 \%$ per year on average. Bariatric surgery (Roux-en-Y gastric bypass surgery, gastric banding, and jejuno-ileal bypass surgery) was found to be more effective in reducing weight and albuminuria. For example, Roux-en-Y gastric bypass surgery reduced body weight by $30 \%$ in a year. Larger weight reduction was accompanied by a greater reduction in hsCRP. However, the effect of bariatric surgery on renal function is inconsistent, due to the reasons discussed above. Of note, according to the statements in 2010 by the Japanese Society for the Surgery of Obesity and Metabolic Disorders (JSSO), surgical intervention is indicated only for morbidly obese subjects with a BMI of over 35 without comorbidity or for those with a BMI of over 32 with comorbidity. In addition, hyperoxaluria after such surgery can cause renal damage and should be prevented by sufficient hydration. Taking these recommendations into consideration, we have concluded that a reduction in body weight and visceral fat mass by restricting energy intake is recommended in subjects with CKD and MetS, at Grade C1.

Several concerns were raised among the working group members. First, it is not clear whether caloric restriction is as safe in subjects with MetS and advanced CKD as in those with MetS without CKD. Second, it is necessary to establish more efficient programs for weight reduction, because of the limited effects of the present lifestyle interventions. Third, the risk of CVD and vitamin deficiency causing conditions such as Wernicke's encephalopathy, should be evaluated carefully during lifestyle interventions.

We have no specific recommendations for subjects with CKD and MetS on target levels and the choice of first line intervention for the other components of MetS at present. As for the specific evidence in MetS subjects, (1) the ARB/ amlodipine combination resulted in anti-diabetic effects compared with the ARB/hydrochlorothiazide combination; (2) the changes in eGFR were better in a strict LDL target group $(<100 \mathrm{mg} / \mathrm{dL})$ than in a moderate LDL target group $(<130 \mathrm{mg} / \mathrm{dL})$; and (3) ezetimibe may have beneficial effects on obesity, hypertension, insulin resistance, and albuminuria.

\section{Bibliography}

1. Agrawal V, et al. Nat Rev Nephrol. 2009;5:520-8. (Level 4)

2. Duran-Perez EG, et al. Metab Syndr Relat Disord. 2011;9:483-89. (Level 4)

3. Bello AK, et al. Nephrol Dial Transplant. 2007;22:1619-27. (Level 4)

4. Afshinnia F, et al. Nephrol Dial Transplant. 2010;25:1173-83. (Level 4)

5. Hofs $\varnothing$ D, et al. Eur J Endocrinol. 2010;163:735-45. (Level 3)

6. Agrawal V, et al. Clin Nephrol. 2008;70:194-202. (Level 4)

7. Schuster DP, et al. Surg Obes Relat Dis. 2011;7:459-64. (Level 4)

8. Agrawal V, et al. Surg Obes Relat Dis. 2009;5:20-6. (Level 4) 
9. Athyros VG, et al. Curr Med Res Opin. 2011;27:1659-68. (Level 2)

10. Yagi S, et al. J Atheroscler Thromb. 2010;17:173-80. (Level 4)

11. Martinez-Martin FJ, et al. J Hum Hypertens. 2011;25:346-53. (Level 2)

CQ3 Is treatment for the metabolic syndrome in patients with CKD recommended to improve their life expectancy?

Because of its anti-albuminuric and potentially renoprotective actions, we recommend a reduction in body weight and visceral fat mass by restricting energy intake in subjects with CKD and MetS, with the exception of premenopausal women and CKD stage G4 and G5. (Grade C1)

There is no definitive evidence from randomized controlled trials demonstrating the effect of intervention for MetS on outcomes in patients with CKD. However, there are three reasons to recommend treatment for MetS in CKD stage G1-G3b through a reduction in body weight, especially in visceral fat mass. First, in CKD stage G1-G3b, several observational studies have shown that MetS, including visceral fat accumulation, is significantly associated with a high risk of CVD morbidity and all-cause mortality. Second, a meta-analysis of observational and interventional studies in CKD (mainly stages G1-G3b) with MetS reported that a reduction in body weight by lifestyle or surgical interventions resulted in decreased systolic BP and albuminuria without causing a significant reduction in GFR. And third, a clinical study in the general population with obesity showed that a significant amount of weight loss by surgical intervention decreased the risk of CVD morbidity and all-cause mortality.

In CKD stages G4-G5, the efficacy of treatment for Mets on mortality remains unknown, because clinical trials evaluating the treatment of MetS have been very limited in these patients. It should be noted that obesity as defined by a higher BMI is not always associated with poorer outcomes in advanced stages of CKD. Reverse epidemiology regarding BMI was repeatedly shown by observational studies in advanced CKD including dialysis patients.

\section{Bibliography}

1. Ramkumar N, et al. Am J Kidney Dis. 2007;49:356-64. (Level 4)

2. Martins D, et al. J Nutr Metab. 2010;Article ID 167162. (Level 4)

3. Iwashima Y, et al. Am J Hypertens. 2010;23:290-8. (Level 4)
4. Agarwal S, et al. Cardiol Res Pract. 2012. doi:10.1155/2012/ 806102. (Level 4)

5. Kramer H, et al. Am J Kidney Dis. 2011;58:177-85. (Level 4)

6. Elsayed EF, et al. Am J Kidney Dis. 2008;52:49-57. (Level 4)

7. Kwan BC, et al. Clin J Am Soc Nephrol. 2007;2:992-8. (Level 4)

8. Obermayr RP, et al. Nephrol Dial Transplant. 2009;24:2421-8. (Level 4)

9. Uusitupa M, et al. PLoS One. 2009;4:e5656. (Level 2)

10. Li G, et al. Lancet. 2008;371:1783-9. (Level 2)

11. Sjöström L, et al. JAMA. 2012;307:56-65. (Level 3)

12. Sjöström L, et al. N Engl J Med. 2007;357:741-52. (Level 3)

13. Johnson DW, et al. Nephrology (Carlton). 2007;12:391-8. (Level 4)

14. Athyros VG, et al. Curr Med Res Opin. 2011;27:1659-68. (Level 4)

15. Navaneethan SD, et al. Clin J Am Soc Nephrol. 2009;4:1565-74. (Level 4)

\section{Chapter 16: Diagnosis of CKD in childhood}

\section{General remarks}

Children under the age of 18 years are the focus of this chapter. In the management of CKD in children, we should always keep in mind when to apply the adult CKD guidelines instead of the pediatric CKD guidelines considering the patient's age and physique.

The etiology and epidemiology of CKD in children

The prevalence of CKD in children is much lower than that in adults. Nonetheless, this important condition is accompanied by many problems that require special attention, including issues that affect growth, development, and school life.

As the relationship between the severity of CKD in children and the degree of proteinuria has not been fully evaluated, a description of the stages of CKD in children should be developed using available knowledge.

The main causes of stage 2 or higher CKD in children are congenital abnormalities of the kidney and urinary tract (CAKUT) or other inherited conditions.

The frequency of CKD is much higher in adults than in children; however, this progressive and intractable condition has devastating effects on a patient's growth, development, and quality of life. Therefore, special attention is needed for proper management in children. 
Table 10 Classification of CKD

\begin{tabular}{llll}
\hline Stage & Description & $\begin{array}{l}\text { GFR }(\mathrm{ml} / \\
\mathrm{min} / \\
\left.1.73 \mathrm{~m}^{2}\right)\end{array}$ & $\begin{array}{c}\text { Classification by } \\
\text { Treatment Status }\end{array}$ \\
\hline $1 \quad$ & $\begin{array}{c}\text { Kidney damage with } \\
\text { normal or increased }\end{array}$ & $\begin{array}{c}\text { 1-5T if kidney } \\
\text { transplant } \\
\text { recipient } \\
\text { GFR }\end{array}$ & $\begin{array}{c}\text { 5D if underlying } \\
\text { dialysis }\end{array}$ \\
2 & $\begin{array}{l}\text { Kidney damage with } \\
\text { mild reduction of GFR }\end{array}$ & $60-89$ & \\
3 & $\begin{array}{l}\text { Moderate reduction of } \\
\text { GFR }\end{array}$ & $30-59$ & \\
4 & Severe reduction of GFR & $15-29$ & \\
5 & Kidney failure & $<15$ & \\
\hline
\end{tabular}

In contrast to adult $\mathrm{CKD}$, there is no reliable evidence available to accurately predict outcomes in children with CKD based on the level of protein or albumin excretion. Therefore, the category " $G$ " or " $A$ " that is used in the classification of adult CKD may not be applicable to CKD in children (Table 10).

Globally, the prevalence of CKD at stage 2 or lower in children is reported to be approximately $18.5-58.3$ cases per million children. Data from a study conducted in Japan indicated that the prevalence of CKD at pre-dialysis stages $3-5$ in patients aged 3 months to 15 years was 29.8 cases per million children, and $91.1 \%$ of these patients had nonglomerular disease, and among them, $68.3 \%$ had CAKUT. The yearly incidence and prevalence of end-stage renal disease (ESRD) in patients aged less than 20 years was reported to be 4 cases per million and 22 cases per million, respectively. CAKUT and hereditary nephropathies $(49.8 \%)$ were the most common causes of ESRD, and glomerulonephritis accounted for $22 \%$ of cases. These findings are similar to those from the North American Pediatric Renal Trials and Collaborative Studies registry and the ItalKid Project from Europe, in which the proportion of patients with CAKUT (48-59 \%) was the largest, while the proportion of glomerulonephritis patients (7-14\%) was not so high.

Overall, the main causes of CKD in children at stage 2 or higher are CAKUT or other inherited conditions. The proportion of ESRD caused by glomerulonephritis was lower in North America and Europe than in a Japanese study conducted in 2008 (22\%). This may be due to the age distribution of the patients, with fewer young patients being treated for ESRD at that time.

\section{Bibliography}

1. Ardissino G, et al. Pediatrics. 2003;111:e382-7. (Level 4)

2. Ishikura K, et al. Nephrol Dial Transplant. 2013 (Epub ahead of print). (Level 4)

3. Hattori S, et al. Pediatr Nephrol. 2002;17:456-61. (Level 4)
Table 11 Normal GFR in children and adolescents

\begin{tabular}{ll}
\hline Age $($ gender $)$ & Mean GFR $\pm \mathrm{SD}\left(\mathrm{mL} / \mathrm{min} / 1.73 \mathrm{~m}^{2}\right)$ \\
\hline 1 week (male and female) & $40.6 \pm 14.8$ \\
2-8 weeks (male and female) & $65.8 \pm 24.8$ \\
$>8$ weeks (male and female) & $95.7 \pm 21.7$ \\
2-12 years (male and female) & $133.0 \pm 27.0$ \\
13-21 years (male) & $140.0 \pm 30.0$ \\
13-21 years (female) & $126.0 \pm 22.0$ \\
\hline
\end{tabular}

Kidney function in children

1. Normal range and variability of the glomerular filtration rate (GFR)

Inulin is the gold standard used for measuring GFR to evaluate kidney function. However, there are no data of inulin-based GFRs according to age and gender in Japanese children. Therefore, the normal range of GFR according to age and gender were adapted using data from foreign countries (Table 11).

\section{Estimated GFR (eGFR)}

Schwartz formula:

\section{Equation 1)}

eGFR $\left(\mathrm{mL} / \mathrm{min} / 1.73 \mathrm{~m}^{2}\right)=\mathrm{k} \times$ body length $(\mathrm{cm}) /$ serum $\mathrm{Cr}$

For the value of the constant $k$, see Table 12; serum creatinine $(\mathrm{Cr})$ was obtained by the Jaffe method.

\section{Equation 2)}

Serum Cr (Jaffe method) $=$ Serum Cr (enzymatic method) +0.2

New Schwartz formula:

Equation 3)

eGFR $\left(\mathrm{mL} / \mathrm{min} / 1.73 \mathrm{~m}^{2}\right)=\mathrm{k} \times$ body length $(\mathrm{cm}) /$ serum $\mathrm{Cr}$

According to the CKiD study, $\mathrm{k}=0.413$; serum $\mathrm{Cr}$ was obtained by the enzymatic method.

eGFR for Japanese children (2-11 years):

\section{Equation 4)}

eGFR $\left(\mathrm{mL} / \mathrm{min} / 1.73 \mathrm{~m}^{2}\right)=\mathrm{k} \times$ body length $(\mathrm{cm}) /$ serum $\mathrm{Cr}$

According to the Japanese Society of Pediatric Nephrology, $\mathrm{k}=0.35$; serum $\mathrm{Cr}$ was obtained by the enzymatic method.

In clinical practice, GFR is usually estimated from creatinine clearance or the serum creatinine concentration. However, the conventional method of measuring renal inulin clearance in children is not feasible in clinical practice because constant intravenous infusion is required. In addition, bladder 
catheterization is sometimes required to ensure adequate urine collection from children, particularly in infants and young children. Moreover, an accurate interpretation of GFR values for individual patients, especially for children and adolescents, requires a clear understanding that the normal level of GFR varies according to age, gender, and body size.

GFR can be estimated from serum creatinine $(\mathrm{Cr})$ in pediatric patients using prediction equations that take into account the patient's height, age, and gender. Among the various prediction formulas that have been developed, the Schwartz formulas are the most widely used (Eq. 1). However, the original Schwartz equation is based on serum $\mathrm{Cr}$ determined by the Jaffe method. This equation may overestimate the GFR if serum $\mathrm{Cr}$ is determined by the enzymatic method. Therefore, serum $\mathrm{Cr}$ should be converted before adopting the Schwartz equation. To convert serum $\mathrm{Cr}$ measured by the enzymatic method to that measured by the Jaffe method, Eq. 2 can be used. Equation 3 is the new Schwartz equation and is an updated equation used to calculate GFR utilizing the enzymatic method. However, the revised formula still overestimates GFR when applied to Japanese children. This may be due to differences in body mass and body height between Japanese and Western children. Recently, the Committee of Measures for CKD in children of the Japanese Society of Pediatric Nephrology established a new formula by measuring inulin clearance in Japanese children aged 2-11 years (Eq. 4, Table 12).

\section{Reference serum creatinine}

For normal children aged 2-11 years:

Equation 5) Median serum $\mathrm{Cr}=0.3 \times$ height $(\mathrm{m})$.

For normal children aged less than 18 years:

Equation 6a) for boys, $y=-1.259 x^{5}+7.815 x^{4}-$ $18.57 \mathrm{x}^{3}+21.39 \mathrm{x}^{2}-11.71 \mathrm{x}+2.628$ and

Equation 6b) for girls, $y=-4.536 x^{5}+27.16 x^{4}-$ $63.47 \mathrm{x}^{3}+72.43 \mathrm{x}^{2}-40.06 \mathrm{x}+8.778$

where $\mathrm{y}$ is the reference serum creatinine level $(\mathrm{mg} / \mathrm{dL})$ and $\mathrm{x}$ is height $(\mathrm{m})$.

Equation 7) eGFR $(\%)=$ (reference serum $\mathrm{Cr} /$ patient's serum $\mathrm{Cr}) \times 100$

Table 12 Constant k for the Schwartz formula

\begin{tabular}{ll}
\hline Age & Constant $\mathrm{k}$ (gender) \\
\hline 1 week & Premature infants 0.33 (male and female) \\
& Term infants 0.45 (male and female) \\
2 weeks-1 year & 0.45 (male and female) \\
2-12 years & 0.55 (male and female) \\
13-21 years & 0.70 (male) \\
& 0.55 (female) \\
\hline
\end{tabular}

Table 13 Serum $\mathrm{Cr}$ distribution in healthy Japanese children (enzymatic method)

\begin{tabular}{|c|c|c|c|c|c|c|}
\hline \multicolumn{2}{|l|}{ Age } & \multicolumn{2}{|l|}{$2.50 \%$} & \multicolumn{2}{|l|}{$50.00 \%$} & $97.50 \%$ \\
\hline \multicolumn{2}{|l|}{ 3-5 (months) } & \multicolumn{2}{|l|}{0.14} & \multicolumn{2}{|l|}{0.2} & 0.26 \\
\hline \multicolumn{2}{|l|}{$6-8$} & \multicolumn{2}{|l|}{0.14} & \multicolumn{2}{|l|}{0.22} & 0.31 \\
\hline \multicolumn{2}{|l|}{ 9-11 } & \multicolumn{2}{|l|}{0.14} & \multicolumn{2}{|l|}{0.22} & 0.34 \\
\hline \multicolumn{2}{|l|}{1 (year) } & \multicolumn{2}{|l|}{0.16} & \multicolumn{2}{|l|}{0.23} & 0.32 \\
\hline \multicolumn{2}{|l|}{2} & \multicolumn{2}{|l|}{0.17} & \multicolumn{2}{|l|}{0.24} & 0.37 \\
\hline \multicolumn{2}{|l|}{3} & \multicolumn{2}{|l|}{0.21} & \multicolumn{2}{|l|}{0.27} & 0.37 \\
\hline \multicolumn{2}{|l|}{4} & \multicolumn{2}{|l|}{0.2} & \multicolumn{2}{|l|}{0.3} & 0.4 \\
\hline \multicolumn{2}{|l|}{5} & \multicolumn{2}{|l|}{0.25} & \multicolumn{2}{|l|}{0.34} & 0.45 \\
\hline \multicolumn{2}{|l|}{6} & \multicolumn{2}{|l|}{0.25} & \multicolumn{2}{|l|}{0.34} & 0.48 \\
\hline \multicolumn{2}{|l|}{7} & \multicolumn{2}{|l|}{0.28} & \multicolumn{2}{|l|}{0.37} & 0.49 \\
\hline \multicolumn{2}{|l|}{8} & \multicolumn{2}{|l|}{0.29} & 0.4 & & 0.53 \\
\hline 9 & & 0.34 & & 0.41 & & 0.51 \\
\hline 10 & & 0.3 & & 0.41 & & 0.57 \\
\hline 11 & & 0.35 & & 0.45 & & 0.58 \\
\hline Age (years) & Male & & & Female & & \\
\hline & $2.50 \%$ & $50.00 \%$ & $97.50 \%$ & $2.50 \%$ & $50.00 \%$ & $97.50 \%$ \\
\hline 12 & 0.4 & 0.53 & 0.61 & 0.4 & 0.52 & 0.66 \\
\hline 13 & 0.42 & 0.59 & 0.8 & 0.41 & 0.53 & 0.69 \\
\hline 14 & 0.54 & 0.65 & 0.96 & 0.46 & 0.58 & 0.71 \\
\hline 15 & 0.48 & 0.68 & 0.93 & 0.47 & 0.56 & 0.72 \\
\hline 16 & 0.62 & 0.73 & 0.96 & 0.51 & 0.59 & 0.74 \\
\hline
\end{tabular}

Although serum $\mathrm{Cr}$ is the most commonly used marker for kidney function, serum $\mathrm{Cr}$ is affected by factors other than GFR, principally $\mathrm{Cr}$ production, which is related to body size and muscle mass. This leads to considerable variability between children of different ages and a relatively wide range of serum $\mathrm{Cr}$ levels in normal individuals. Therefore, the Committee of Measures for CKD in Children of the Japanese Society of Pediatric Nephrology established a normal reference value of serum $\mathrm{Cr}$ for healthy Japanese children in 2011 (Table 13).

For children aged 2-11 years, the reference serum $\mathrm{Cr}$ level can be estimated using a simple equation (Eq. 5). For all children under 18 years of age, the individual serum $\mathrm{Cr}$ reference value can be estimated using a polynomial formula (Eq. 6). By combining equations 5 and 6, kidney function (\%) in children aged 2-11 years can be estimated by using Eq. 7.

\section{Bibliography}

1. Heilbron DC, et al. Pediatr Nephrol. 1991;5:5-11. (Level 4)

2. Coulthard MG. Early Hum Dev. 1985;11:281-92. (Level 4)

3. Schwartz GJ, et al.J Pediatr. 1984;104:849-54. (Level 4)

4. Schwartz GJ, et al.Pediatrics. 1976;58:259-63. (Level 4)

5. Brion LP, et al. J Pediatr. 1986;109:698-707. (Level 4)

6. Schwartz GJ, et al. J Am Soc Nephrol. 2009;20:629-37. (Level 4) 
7. Nagai T, et al. Clin Exp Nephrol. 2013 (Epub ahead of print). (Level 4)

8. Uemura O, et al. Clin Exp Nephrol. 2011;15:694-9. (Level 4)

CQ1 Are the definition and staging of CKD in children the same as in adults?

The diagnosis of CKD in children is based on the same definition as that used for CKD in adults; however, proteinuria is excluded from CKD classification in children.

\section{Definition of CKD in children}

The same definition for adult CKD is used to diagnose children.

\section{Classification of CKD in children}

In adults, the degree of proteinuria is also included in the staging of CKD based on data that showed correlation between the level of proteinuria and the prognosis. However, the degree of proteinuria in children is not as clearly correlated with the prognosis. Proteinuria is observed only in rare cases of CAKUT, the most common cause of stage 5 $\mathrm{CKD}$ in children. Moreover, there are no significant data that suggest a relationship between kidney function and the degree of proteinuria in children. Hence, proteinuria is not currently used to classify CKD in children and the notations "G (= GFR)" and "A (= Albuminuria)," which are used in adult CKD staging, are not applied to CKD staging in children (Table 10).

Children under 2 years of age typically have a low GFR even after correcting for body surface area. Therefore, the aforementioned classification cannot be used for very young patients. Alternatively, a calculated GFR value based on serum creatinine can be compared with the normal age-appropriate values to detect kidney impairment.

\section{Bibliography}

1. Heilbron DC, et al. Pediatr Nephrol. 1991;5:5-11. (Level 4)

2. Coulthard MG. Early Hum Dev. 1985;11:281-92. (Level 4)

3. Schwartz GJ, et al. J Pediatr. 1984;104:849-54. (Level 4)

4. Rhodin MM, et al. Pediatr Nephrol. 2009;24:67-76. (Level 4)

5. Uemura O, et al. Clin Exp Nephrol. 2011;15:694-9. (Level 4)

6. Wong CS, et al. Clin J Am Soc Nephrol. 2009;4:812-9. (Level 4)
CQ2 Would a urinary screening program among school children be useful for improving the prognosis of CKD in children?

A urinary screening program among school children may contribute to the early detection of CKD and can improve the prognosis, especially for patients with chronic glomerulonephritis.

Since 1974, a urinary screening program has been performed for all school children annually, which has contributed to the early detection of CKD in children in Japan.

The prevalence of hematuria, proteinuria, and both abnormalities are approximately $0.75,0.16$, and $0.04 \%$, respectively, in elementary school children and approximately $0.98,0.53$, and $0.1 \%$, respectively, in junior high school students in Japan. Most children with chronic glomerulonephritis are identified by the urinary screening program at stage 1 CKD.

The outcomes of patients with membranoproliferative glomerulonephritis in Japan were superior to the findings of other studies due to earlier detection and treatment. Among children diagnosed with type I membranoproliferative glomerulonephritis by the screening program, no child developed ESRD. Furthermore, among the children who have been undergoing the annual school-screening program, the age at which ESRD developed has been increasing. Thus, urinary screening would not only help in early detection, but consequently also with preventing the deterioration of renal function later in life. However, controversy about the usefulness of screening still exists, particularly regarding the cost-effectiveness of screening asymptomatic subjects.

\section{Bibliography}

1. Murakami M, et al. Pediatr Nephrol. 1991;5:50-3. (Level 4)

2. Murakami M, et al. Kidney Int. 2005;94(Suppl):S23-7. (Level 4)

3. Kamei K, et al. Clin J Am Soc Nephrol. 2011;6:1301-7. (Level 2)

4. Yanagihara T, et al. Pediatr Nephrol. 2005;20:585-90. (Level 4)

5. Cho BS, et al. Pediatr Nephrol. 2001;16:1126-8. (Level 4)

6. Lee YM, et al. Acta Paediatr. 2006;95:849-53. (Level 4)

7. Park YH, et al. Pediatr Nephrol. 2005;20:1126-30. (Level 4)

8. Lin CY, et al. Pediatr Nephrol. 2001;16:232-37. (Level 4)

9. Yamagata K, et al. Am J Kidney Dis. 2004;43:433-43. (Level 4) 
CQ3 Is hematuria useful for detecting CKD

in children?

Hematuria is an important symptom of CKD in children. Gross hematuria is a particularly important symptom that often suggests the presence of disease with a poor prognosis.

Asymptomatic isolated microscopic hematuria is the most common presentation of microscopic hematuria, and most pediatric Japanese patients are discovered using the urinary screening program. This disease is usually transient and does not require treatment. Asymptomatic isolated microscopic hematuria is present in $0.75-0.98 \%$ of schoolaged children in Japan. The most common causes of persistent microscopic hematuria include glomerulopathies, hypercalciuria, and the nutcracker syndrome. Glomerulopathies include IgA nephropathy, hereditary nephritis (Alport syndrome), and thin basement membrane nephropathy. Lupus nephritis is often associated with severe glomerular damage even with asymptomatic microscopic hematuria. CAKUT, the most common cause of ESRD in children, is also associated with microscopic hematuria. Thus, microscopic hematuria should always be considered as a potential underlying symptom of these critical kidney diseases.

The relative incidence of the known causes of gross hematuria in children varies depending upon the clinical setting. In a pediatric emergency room, a urinary tract infection, either documented or suspected, was diagnosed in half of the patients with gross hematuria. Other causes included urethral irritation $(11 \%)$, trauma $(7 \%)$, and acute nephritis (4\%). In a pediatric urology referral service, the causes of gross hematuria and their frequencies included urethral irritation or trauma (15\%), urinary tract infection (14\%), underlying congenital anomalies (13\%), nephrolithiasis (5\%), and malignancy (1\%). There were no cases of glomerular disease. By contrast, at a pediatric nephrology referral service, a glomerular cause was identified in about $30 \%$ of patients. Of note, a non-glomerular etiology was established in $37 \%$ of patients. The most common diagnosis was hypercalciuria. Of note, CAKUT, the most common cause of ESRD in children, was diagnosed in $3.5 \%$ of those patients. Malignancies (Wilms' tumors or transitional cell carcinoma of the bladder) are also important causes of gross hematuria, but are much less common in children than in adults. To investigate the causes of hematuria, urine sediment examination and imaging studies are necessary.

\section{Bibliography}

1. Murakami M, et al. Pediatr Nephrol. 1991;5:50-3. (Level 4)

2. Dodge WF, et al. J Pediatr. 1976;88:327-47. (Level 4)

3. Vehaskari VM, et al. J Pediatr. 1979;95:676-84. (Level 4)

4. Bergstein J, et al. Arch Pediatr Adolesc Med. 2005;159:353-5. (Level 4)

5. Greenfield SP, et al. Urology. 2007;69:166-9. (Level 4)

6. Ingelfinger JR, et al. Pediatrics. 1977;59:557-61. (Level 4)

7. Park YH, et al. Pediatr Nephrol. 2005;20:1126-30. (Level 4)

8. Okada M, et al. Clin Nephrol. 1998;49:35-40. (Level 4)

9. Lee YM, et al. Acta Paediatr. 2006;95:849-53. (Level 4)

10. Schröder CH, et al. Acta Paediatr Scand. 1990;79:630-6. (Level 4)

CQ4 Is renal biopsy useful for the diagnosis and treatment of CKD in children?

Renal biopsy is useful for making a diagnosis, assessing disease severity, and deciding on the treatment of CKD in children.

Renal biopsy is recommended for cases with persistent proteinuria (urinary protein-to-creatinine ratio: $\geq 0.5 \mathrm{~g} / \mathrm{gCr}, \geq 3$ months; aged 2 years or older) and persistent hematuria + proteinuria (hematuria + urinary protein-to-creatinine ratio $: \geq 0.2 \mathrm{~g} / \mathrm{gCr}, \geq 3$ months; aged 2 years or older).

Renal biopsy is recommended for the following cases:

1. Persistent proteinuria (urinary protein-to-creatinine ratio: $\geq 0.5 \mathrm{~g} / \mathrm{gCr}, \geq 3$ months; aged 2 years or older)

2. Persistent hematuria + proteinuria (hematuria + urinary protein-to-creatinine ratio: $\geq 0.2 \mathrm{~g} / \mathrm{gCr}$, $\geq 3$ months; aged 2 years or older)

3. Nephrotic syndrome: unlike adults, renal biopsy is not indicated for most children with nephrotic syndrome.

The following cases are exceptional in childhood nephrotic syndrome, and renal biopsy is recommended: cases in which underlying diseases other than minimal change nephrotic syndrome are suspected, cases which are suspected to be congenital nephrotic syndrome, or cases of steroid-resistant nephrotic syndrome.

4. Rapidly progressive glomerulonephritis syndrome

5. Systemic lupus erythematosus (SLE)

6. Henoch-Schönlein purpura nephritis with nephrotic syndrome, acute nephritic syndrome, rapidly progressive glomerulonephritis syndrome, or cases with persistent proteinuria.

The usefulness of renal biopsies has been supported in some cohort studies to evaluate the Oxford IgA nephropathy classification, the International Society of 
Nephrology/Renal Pathology Society (ISN/RPS) 2003 Classification of Lupus Nephritis, and other some clinicopathological studies.

\section{Bibliography}

1. Coppo R, et al. Kidney Int. 2010;77:921-7. (Level 4)

2. Ninchoji T, et al. Pediatr Nephrol. 2011;26:563-9. (Level 4)

3. Wakaki H, et al. Pediatr Nephrol. 2011;26:921-5. (Level 4)

4. Marks SD, et al. Pediatr Nephrol. 2007;22:77-83. (Level 4)

5. Askenazi D, et al. Pediatr Nephrol. 2007;22:981-6. (Level 5)

6. Abrantes MM, et al. Pediatr Nephrol. 2006;21:1003-12. (Level 4)

7. Paik KH, et al. Pediatr Nephrol. 2007;22:389-95. (Level 4)

8. Arias LF, et al. Nephrol Dial Transplant. 2011;26:2215-21. (Level 4)

9. Hama T, et al. Nephrol Dial Transplant. 2012;27:3186-90. (Level 4)

10. Sellers EA, et al. Diabetes Care. 2009;32:786-90. (Level 4)

CQ5 Are imaging studies useful for the diagnosis and treatment of CKD in children?

Imaging studies (abdominal ultrasound, MRI, and etc.) are important for the diagnosis of CKD in children. Imaging studies are particularly important for making a diagnosis and deciding on the treatment for CAKUT.

Imaging studies are performed for patients fitting one of the following criteria: presenting with (1) abdominal pain and masses, (2) urinary tract infection, or (3) CKD including abnormal urinary findings.

Imaging studies are useful for detecting the following diseases: (1) obstructive nephropathy, (2) reflux nephropathy, (3) dysplastic/hypoplastic kidney, (4) solitary kidney, horseshoe kidney, (5) floating kidney, and (6) cystic kidney disease.

For the examination of vesicoureteral reflux, an initial screening via ultrasound is important for patients with hydronephrosis or urinary tract infection. Avoiding cystourethrogram is recommended for patients with abnormalities on a renal ultrasound or who develop a UTI during observation.

\section{Bibliography}

1. Marks SD, et al. Pediatr Nephrol. 2008;23:9-17. (Level 5)

2. Skoog SJ, et al. J Urol. 2010;184:1145-51. (Level 4)

3. Yang H, et al. Nephrology. 2010;15:362-7. (Level 4)

4. Tsuchiya M, et al. Pediatr Int. 2003;45:617-23. (Level 4)

5. Vester U, et al. Pediatr Nephrol. 2010;25:231-40. (Level 5)

6. Morales Ramos DA, et al. Curr Probl Diagn Radiol. 2007;36:153-63. (Level 5)
CQ6 Is a differential renal function test useful for the diagnosis and treatment of CKD in children?

A differential renal function test is useful for the diagnosis and treatment of CKD in children. Dynamic radionuclide scans to assess the renal excretory function with $99 \mathrm{mTc}-$ mercaptotriglycylglycine (MAG3) is useful for the diagnosis of and decision to perform surgical intervention for CAKUT, particularly in cases with obstructive uropathy.

There are not enough studies that have evaluated the differential renal function test for CKD in children and further studies are required to assess its usefulness.

\section{Bibliography}

1. Marks SD, et al. Pediatr Nephrol. 2008;23:9-17. (Level 5)

2. Ritchie G, et al. Pediatr Radiol. 2008;38:857-62. (Level 5)

3. Ross SS, et al. J Pediatr Urol. 2011;7:266-71. (Level 4)

4. Schlotmann A, et al. Eur J Nucl Med Mol Imaging. 2009;36:1665-73. (Level 4)

5. Aktas GE, Inanir S. Ann Nucl Med. 2010;24:691-5. (Level 4)

Q7 Is CKD in children a risk for end-stage kidney disease?

The deterioration of GFR in children is a risk factor for end-stage kidney disease (ESKD).

As GFR deterioration is related to urine protein excretion and hypertension, active intervention for these conditions is necessary to prevent ESKD even in children.

We reviewed previous reports about CKD in children and concluded that CKD in children is a risk factor for ESKD, as well as for adults. The positive finding of a significant correlation between GFR deterioration and urinary protein excretion suggested that even children at an earlier stage of CKD are at risk for ESKD. Moreover, strict management of blood pressure has been demonstrated to suppress GFR deterioration in pediatric CKD.

Note that the rate of decrease in GFR for cases with CAKUT and VUR is generally slower than in those with glomerular diseases. 


\section{Bibliography}

1. Soares CM, et al. Nephrol Dial Transplant. 2009;24:848-55. (Level 4)

2. ESCAPE Trial Group, et al. N Engl J Med. 2009;361:1639-50. (Level 2)

3. Mong Hiep TT, et al. Pediatr Nephrol. 2010;25:935-40. (Level 4)

4. Staples AO, et al. Clin J Am Soc Nephrol. 2010;5:2172-9. (Level 4)

5. Wong CS, et al. Clin J Am Soc Nephrol. 2009;4:821-9. (Level 4)

6. Furth SL, et al. Pediatr Nephrol. 2007;22:265-71. (Level 4)

7. Abitbol CL, et al. Pediatr Nephrol. 2009;24:1363-70. (Level 4)

8. Vikse BE, et al. J Am Soc Nephrol. 2008;19:151-7. (Level 4)

9. Ardissino G, et al. Pediatrics. 2003;111:e382-7. (Level 4)

10. Furth SL, et al. Clin J Am Soc Nephrol. 2011;6:2132-40. (Level 4)

11. Novak TE, et al. J Urol. 2009;182:1678-81. (Level 4)

CQ8 Is CKD in children a risk for cardiovascular disease?

Deterioration of GFR in children is a risk factor for cardiovascular disease (CVD). It is crucial for children with CKD to control their blood pressure in order to prevent the occurrence of CVD.

We reviewed previous reports about CKD in children and concluded that CKD in children is a risk factor for CVD. On the other hand, it is notable that there are few pediatric patients with coronary artery or cerebrovascular disease, which are frequent in adults with CKD.

It is crucial to control blood pressure, which is a traditional CVD risk factor. Some previous reports suggested that the target value of blood pressure for children with CKD should be lower than that for healthy children. Nontraditional CVD risk factors for CKD in children are still being investigated.

\section{Bibliography}

1. Parekh RS, et al. J Pediatr. 2002;141:191-7. (Level 4)

2. Groothoff JW, et al. Kidney Int. 2002;61:621-9. (Level 4)

3. Chavers BM, et al. Kidney Int. 2002;62:648-53. (Level 4)

4. Mitsnefes M, et al. J Am Soc Nephrol. 2003;14:2618-22. (Level 4)

5. Wong H, et al. Kidney Int. 2006;70:585-90. (Level 4)

6. Furth SL, et al. Clin J Am Soc Nephrol. 2011;6:2132-40. (Level 4)

7. Sinha MD, et al. Clin J Am Soc Nephrol. 2011;6:543-51. (Level 4)

8. Rinat C, et al. Nephrol Dial Transplant. 2010;25:785-93. (Level 4)

9. Oh J, et al. Circulation. 2002;106:100-5. (Level 4)
CQ9 Is CKD in children a risk for growth impairment?

Deterioration of GFR in children is a risk factor for growth impairment. Children with CKD can be suitable candidates for replacement therapy with growth hormone. It is of great importance for children with CKD to have good nutrition in order to prevent growth impairment.

Some previous reports demonstrated that $10-40 \%$ of CKD in children, including ESKD, were associated with a short stature. The physical condition associated QOL of CKD in children with a short stature is significantly lower than that of healthy children. Moreover, pediatric cases of CKD with a severely short stature have been shown to have a higher risk of hospitalization and mortality.

Children with CKD are indicative of resistance to growth hormone and insulin-like growth factor. Accordingly, children with CKD are suitable candidates for replacement therapy with growth hormone. Additionally, it is crucial to provide good nutrition especially in infancy and early childhood.

\section{Bibliography}

1. Wong H, et al. Kidney Int. 2006;70:585-90. (Level 4)

2. Seikaly MG, et al. Pediatr Nephrol. 2006;21:793-799. (Level 4)

3. Wada N, Syouni PD. Kenkyuukaishi. 2000;13:32-5. (Level 4)

4. Furth SL, et al. Pediatr Nephrol. 2002;6:450-5. (Level 4)

5. Gerson AC, et al. Pediatrics. 2010;125:e349-457. (Level 4)

6. Furth SL, et al. Clin J Am Soc Nephrol. 2011;6:2132-40. (Level 4)

7. Kari JA, et al. Kidney Int. 2001;57:1681-7. (Level 4)

\section{Chapter 17: Management of CKD in childhood}

Treatment for IgA nephropathy in children

\section{Background}

The optimal approach for treating pediatric IgA nephropathy should be determined from the histological findings. Mild IgA nephropathy is histologically defined as focal mesangial proliferation. Severe IgA nephropathy is histologically defined as diffuse mesangial proliferation or more than $50 \%$ of the glomeruli containing crescents.

\section{Treatment for mild IgA nephropathy}

We recommend ACE inhibitors as the first choice of agent for treating mild IgA nephropathy, because they reduce urinary protein excretion and inhibit the progression of IgA nephropathy. We suggest that ARBs are useful for treating mild IgA nephropathy, because they may reduce 
urinary protein excretion. Currently available evidence does not support the conclusion that combination therapy with an ACE inhibitor and an ARB is essential in the treatment of mild IgA nephropathy. Therefore, we do not recommend combination therapy with an ACE inhibitor and an ARB for treating mild IgA nephropathy. The physician should decide on the doses of an ACE inhibitor or an ARB with reference to the doses used as antihypertensive agents for children (Section 17 CQ5). The physician should start with low doses of an ACE inhibitor or an ARB and increase the dose while carefully monitoring the patient for side effects.

\section{Treatment for severe $\operatorname{IgA}$ nephropathy}

We recommend combined therapy with prednisolone, an immunosuppressive agent (azathioprine or mizoribine), warfarin and dipyridamole for 2 years for severe IgA nephropathy (Table 14). Two RCTs and one clinical trial in pediatric patients with severe $\operatorname{IgA}$ nephropathy have demonstrated that this regimen can reduce urinary protein excretion and inhibit the progression of glomerular sclerosis. Two cohort studies have demonstrated that this regimen can improve the long-term prognosis of children with severe IgA nephropathy.

\section{Tonsillectomy for IgA nephropathy}

Reports of tonsillectomy in children have come from predominantly retrospective studies and have not included adequate controls. It is difficult to interpret the data, because most of the patients reported in these studies also received concomitant medications, such as corticosteroids. We recommend that a conservative approach be maintained for children with recurrent gross hematuria unless they have additional risk factors, including a history of frequent episodes of tonsillitis or massive proteinuria.

Table 14 Combined therapy for 2 years

(1) Prednisolone

(2) Immunosuppressive agent

Oral administration of $2 \mathrm{mg} / \mathrm{kg}$ per dose (max $100 \mathrm{mg}$ ) of azathioprine one time per day or $4 \mathrm{mg}$ per dose (max $150 \mathrm{mg}$ ) of mizoribine one or two times per day

(3) Warfarin

Oral administration of warfarin one time per day. Regulate the dose of warfarin using the thrombo test with a target range of $20-50 \%$

(4) Dipyridamole

Start oral administration of $3 \mathrm{mg} / \mathrm{kg}$ per dose of dipyridamole three times per day; if there are no side effects, increase the dose to $6-7 \mathrm{mg} / \mathrm{kg}$ per dose (max $300 \mathrm{mg}$ )

\section{Bibliography}

1. Yata N, et al. Pediatr Nephrol. 2008;23:905-12. (Level 4)

2. Coppo R, et al. J Am Soc Nephrol. 2007;18:1880-8. (Level 2)

3. Nakanishi K, et al. Pediatr Nephrol. 2009;24:845-9. (Level 4)

4. Ellis D, et al. J Pediatr. 2003;143:89-97. (Level 4)

5. Bhattacharjee R, et al. Eur J Pediatr. 2000;159:590-3. (Level 5)

6. Yang Y, et al. Clin Nephrol. 2005;64:35-40. (Level 5)

7. Yoshikawa N, et al. J Am Soc Nephrol. 1999;10:101-9. (Level 2)

8. Yoshikawa N, et al. Clin J Am Soc Nephrol. 2006;1:511-7. (Level 2)

9. Yoshikawa N, et al. Pediatr Nephrol. 2008;23:757-63. (Level 4)

10. Kamei K, et al. Clin J Am Soc Nephrol. 2011;6:1301-7. (Level 4)

11. Kawasaki Y, et al. Pediatr Nephrol. 2006;21:701-6. (Level 2)

Treatment for nephrotic syndrome in children (including focal segmental glomerulosclerosis-FSGS)

\section{Background}

Corticosteroid therapy can be initiated without histological confirmation from a renal biopsy because most patients with idiopathic pediatric nephrotic syndrome (NS) respond well to corticosteroids. However, a renal biopsy and histological diagnosis are recommended before starting steroid therapy in the following cases: (1) patient younger than 1 year old; (2) apparent hematuria; (3) hypertension or elevated serum creatinine levels; (4) extra-renal symptoms, such as rash or purpura; and (5) hypocomplementaemia.

\section{Initial treatment for NS}

We recommend the standard initial treatment regimen ( 2 months) proposed by the International Study of Kidney Diseases in Children (the ISKDC regime) or longer initial regimens (3-7 months) for the initial corticosteroid treatment. Although a meta-analysis demonstrated that the risk of relapse at 12-24 months was reduced by $30 \%$ (risk ratio of relapse $0.70 ; 95 \%$ CI $0.58-0.84$ ) with longer initial regimens compared to the ISKDC regime, the optimum dose and duration of the initial treatment has not yet been determined.

\section{Treatment for relapsing NS}

There have been no RCTs examining corticosteroid therapy for relapsing NS. We recommend the administration of corticosteroid therapy according to the ISKDC method: prednisone at $60 \mathrm{mg} / \mathrm{m}^{2}$ per day until urine protein tests become negative for three consecutive days, followed by $60 \mathrm{mg} / \mathrm{m}^{2}$ on alternate days for 2 weeks, then $30 \mathrm{mg} / \mathrm{m}^{2}$ on alternate days for 2 weeks, and, finally, $15 \mathrm{mg} / \mathrm{m}^{2}$ on alternate days for 2 weeks. 
4. Treatment for frequent relapsing/steroid-dependent NS (FRNS/SDNS)

We recommend cyclosporine or cyclophosphamide for FRNS/SDNS treatment. Cyclosporine is effective for inducing or maintaining remission in patients with FRNS or SDNS. Cyclosporine may have significant adverse effects including chronic nephrotoxicity and posterior reversible leukoencephalopathy syndrome. Receiving cyclosporine for 24 months or more and at high doses (C2 levels $>600 \mathrm{ng} / \mathrm{mL}$ ) are risk factors for chronic nephrotoxicity. Cyclophosphamide can induce longer lasting remissions than prednisone alone in FRNS patients. The effect of cyclophosphamide on SDNS may depend upon the duration of treatment and the degree of steroid dependency and may affect remission rates. Cyclophosphamide may have significant adverse effects, including bone marrow suppression, gonadal toxicity and malignancy. The development of gonadal toxicity resulting in infertility generally is caused by a total cyclophosphamide dose greater than $300 \mathrm{mg} / \mathrm{kg}$. Further studies, including controlled trials, are needed to determine the efficacy and safety of mycophenolate mofetil and rituximab for children with refractory FRNS/SDNS.

\section{Treatment for steroid-resistant NS (SRNS)}

We recommend cyclosporine and/or steroid pulse therapy for SRNS treatment. Cyclosporine is effective in inducing remission in patients with SRNS. A small study suggested that cyclosporine and steroid pulse therapy for Japanese patients with focal segmental glomerulosclerosis may be effective in inducing remission.

\section{Bibliography}

1. International Study of Kidney Disease in Children. J Pediatr. 1981;98:561-4. (Level 4)

2. Tarshish P, et al. J Am Soc Nephrol. 1997;8:769-76. (Level 4)

3. Cattran DC, et al. Am J Kidney Dis. 1998;32:72-9. (Level 5)

4. Ueda N, et al. J Pediatr. 1988;112:122-6 (Level 2 per protocol analysis).

5. Ehrich JH, et al. Eur J Pediatr. 1993;152:905-12 (Level 2 per protocol analysis).

6. Ksiazek J, et al. Acta Pediatr. 1995;84:889-93 (Level 2 per protocol analysis).

7. Bagga A, et al. Pediatr Nephrol. 1999;13:824-7 (Level 2 per protocol analysis).

8. Hiraoka M, et al. Am J Kidney Dis. 2003;41:1155-62. (Level 2)

9. Ishikura K, et al. Kidney Int. 2008;73:1167-73. (Level 2)

10. Ishikura K, et al. Nephrol Dial Transplant. 2010;25:3956-62. (Level 4)

11. Niaudet P, et al. J Am Soc Nephrol. 1994;4:1049-56. (Level 4)

12. Ishikura K, et al. Clin J Am Soc Nephrol. 2012;7:1573-83. (Level 4)

13. Iijima K, et al. Kidney Int. 2002;61:1801-5. (Level 4)

14. Kengne-Wafo S, et al. Clin J Am Soc Nephrol. 2009;4:1409-16. (Level 5)
15. Ponticelli C, et al. Nephrol Dial Transplant. 1933;8:1326-32. (Level 2)

16. Arbeitsgemeinschaft für Pädiatrische Nephrologie. N Engl J Med. 1982;306:451-4. (Level 2)

17. Zagury A, et al. Pediatr Nephrol. 2011;26:915-20. (Level 4)

18. Arbeitsgemeinschaft für Pädiatrische Nephrologie. Arch Dis Child. 1987;62:1102-6. (Level 3)

19. Latta K, et al. Pediatr Nephrol. 2001;16:271-82. (Level 4)

20. Bagga A, et al. Am J Kidney Dis. 2003;42:1114-20. (Level 4)

21. Novak I, et al. Pediatr Nephrol. 2005;20:1265-8. (Level 4)

22. Hogg RJ, et al. Clin J Am Soc Nephrol. 2006;1:1173-8. (Level 4)

23. Fujinaga S, et al. Pediatr Nephrol. 2007;22:71-6. (Level 4)

24. Afzal K, et al. Pediatr Nephrol. 2007;22:2059-65. (Level 4)

25. Dorresteijn EM, et al. Pediatr Nephrol. 2008;23:2013-20 (Level 2 per protocol analysis).

26. Guigonis V, et al. Pediatr Nephrol. 2008;23:1269-79. (Level 4)

27. Kamei K, et al. Pediatr Nephrol. 2009;24:1321-8. (Level 4)

28. Prytuła A, et al. Pediatr Nephrol. 2010;25:461-8. (Level 5)

29. Sellier-Leclerc AL, et al. Pediatr Nephrol. 2010;25:1109-15. (Level 4)

30. Gulati A, et al. Clin J Am Soc Nephrol. 2010;5:2207-12. (Level 4)

31. Ravani P, et al. Clin J Am Soc Nephrol. 2011;6:1308-15 (Level 2 per protocol analysis).

32. Hamasaki Y, et al. Pediatr Nephrol. 2009;24:2177-85. (Level 4)

33. Ehrich JH, et al. Nephrol Dial Transplant. 2007;22:2183-93. (Level 4)

34. Mori K, et al. Pediatr Nephrol. 2004;19:1232-6. (Level 5)

CQ1 Is restriction of exercise recommended to slow the progression of renal dysfunction in children with CKD?

Restriction of exercise is not recommended for children with CKD because it is not evident whether it can slow the progression of renal dysfunction. (Grade C2)

It is well known that exercise causes a transient increase in urinary protein excretion and that bed rest decreases urinary protein excretion in CKD. However, it is unknown how these phenomena affect the progression of renal dysfunction in the long term. This CQ aims to determine whether exercise or restriction of exercise have any effect on the progression of renal dysfunction in children with CKD.

It is not evident that exercise has an effect on the progression of renal dysfunction in children with CKD. Several studies have reported that exercise only transiently altered GFR and urine protein excretion in CKD, and that long-term restriction of exercise did not significantly affect creatinine clearance and urinary findings in mild to moderate $\operatorname{IgA}$ nephropathy and non-IgA mesangial proliferative glomerulonephritis in children. Therefore, restriction of exercise is not recommended for children with chronic 
glomerulonephritis with only mild proteinuria and stable renal function or children with nephrotic syndrome in remission. However, it is unknown whether or not longterm, heavy exercise has an effect on renal function and whether exercise has an effect on heavy-proteinuric chronic glomerulonephritis and focal segmental glomerulosclerosis. Restriction of exercise is necessary in patients with prominent edema, refractory hypertension, or congestive heart failure, and in patients receiving anticoagulant therapy. On the other hand, it should also be noted that excessive restriction of exercise can cause severe adverse effects, such as substantial psychological stress resulting in a decreased QOL as well as aggravation of obesity; furthermore, osteoporosis induced by corticosteroid therapy can result in a vertebral compression fracture. In conclusion, restriction of exercise should be considered with caution based on a comprehensive evaluation of these circumstances in individual patients.

\section{Bibliography}

1. Ito K. J Jpn Pediatr Soc. 1989;93:875-83. (Level 4)

2. Furuse A, et al. J Jpn Pediatr Soc. 1989;93:884-9. (Level 4)

3. Taverner D, et al. Nephron. 1991;57:288-92. (Level 4)

4. Nagasaka Y. Nihon Jinzo Gakkai Shi. 1986;28:1465-70. (Level 4)

5. Fuiano G, et al. Am J Kidney Dis. 2004;44:257-63. (Level 4)

6. Furuse A, et al. Nihon Jinzo Gakkai Shi. 1991;33:1081-7. (Level 3)

7. Nagasaka Y, et al. J Jpn Pediatr Soc. 1986;90:2737-41. (Level 4)

\section{CQ2 Is dietary protein restriction recommended}

to slow the progression of renal dysfunction for children with CKD?

Dietary protein restriction is not recommended for children with CKD because it is not evident whether it can slow the progression of renal dysfunction. (Grade C2)
While several reports have suggested that dietary protein restriction can protect renal function in adults with CKD, few articles have been published in the field of children with CKD. This CQ aims to determine the efficacy of a protein restricted diet in delaying the progression to end-stage kidney disease and its impact on growth in children.

Several RCTs have shown that protein restriction is not effective to slow the progression of renal dysfunction in children with CKD. Considering the recommendation of the KDOQI guidelines, it is reasonable to assume that the target level of dietary protein intake in children with CKD should follow the Recommendation for Japanese Dietary Intakes by the Ministry of Health, Labor and Welfare (Table 15). However, it should be noted that this recommendation means a virtual protein restriction because spontaneous dietary protein intake in children with CKD is far in excess of the average requirements, typically $150-200 \%$ of the recommended dietary allowance. In addition, protein restriction may have a beneficial effect on renal dysfunction in children if adequate nutritional management is provided by a dietitian who has expertise in pediatric and renal nutrition. It should also be noted that protein restriction is necessary to control hyperphosphatemia and severe azotemia in advanced CKD, as it ameliorates blood urea nitrogen/creatinine ratios. In regard to growth, there was no significant difference in height between the protein-restricted versus control groups in most relevant RCTs.

\section{Bibliography}

1. Uauy RD, et al. Pediatr Nephrol. 1994;8:45-50. (Level 2)

2. Kist-van Holthe tot Echten JE, et al. Arch Dis Child. 1993;68:371-5. (Level 2)

3. Hattori M, et al. J Jpn Pediatr Soc. 1992;96:1046-57. (Level 4)

4. Jureidini KF, et al. Pediatr Nephrol. 1990;4:1-10. (Level 4)

5. Wingen AM, et al. Lancet. 1997;349:1117-23. (Level 2)
Table 15 Protein intake in children (g/day) from The Recommendation for Japanese Dietary Intakes 2010 (http:// www.mhlw.go.jp/bunya/ kenkou/sessyu-kijun.html)

\begin{tabular}{|c|c|c|c|c|}
\hline \multirow[t]{2}{*}{ Age } & \multicolumn{2}{|l|}{ Boys } & \multicolumn{2}{|l|}{ Girls } \\
\hline & Recommended amount & Adequate amount & Recommended amount & Adequate amount \\
\hline $0-5$ months & & 10 & & 10 \\
\hline $6-8$ months & & 15 & & 15 \\
\hline 9-11 months & & 25 & & 25 \\
\hline $1-2$ years & 20 & & 20 & \\
\hline $3-5$ years & 25 & & 25 & \\
\hline $6-7$ years & 30 & & 30 & \\
\hline $8-9$ years & 40 & & 40 & \\
\hline $10-11$ years & 45 & & 45 & \\
\hline $12-17$ years & 60 & & 55 & \\
\hline
\end{tabular}


CQ3 Is salt restriction recommended to slow the progression of renal dysfunction in children with CKD?

Salt restriction may be recommended for children with CKD having hypertension because it is effective at lowering blood pressure, which in turn, may result in slowing the progression of renal dysfunction. (Grade C1)

Salt intake should not be restricted in children with CKD having polyuric, salt-wasting forms of congenital anomalies of the kidney and the urinary tract. (Grade D)

Salt restriction is recommended for adult CKD with and without hypertension because it reduces urinary protein excretion and protects the renal function in adult CKD. In children, the major cause of CKD is congenital anomalies of the kidney and the urinary tract (CAKUT) with polyuric, salt-wasting nephropathy. This CQ aims to determine if salt restriction slows the progression of renal dysfunction in pediatric CKD and if sodium and water supplementation has beneficial effects on polyuric, salt-wasting forms of CAKUT.

There have been no reports of studies investigating whether or not salt restriction per se slows the progression of renal dysfunction and reduces urinary protein excretion in children with CKD with or without hypertension. However, it has been shown that strict blood-pressure control confers a substantial benefit with respect to renal function among children with CKD (CQ5). Several RCTs have shown that salt restriction is effective in lowering blood pressure in children in the general population both in the short and long term. Taken together, salt restriction may be effective in lowering blood pressure in children with CKD, which would result in slowing the progression of renal dysfunction. On the other hand, some cohort studies have shown that nutritional support with sodium and water supplementation can maintain or improve the growth of children with polyuric, salt-wasting CKD. Therefore, salt intake should not be restricted in children with polyuric, salt-wasting forms of CAKUT.

\section{Bibliography}

1. He FJ, et al. Hypertension. 2006;48:861-9. (Level 1)

2. He FJ, et al. J Hum Hypertens. 2008;22:4-11. (Level 4)

3. Geleijnse JM, et al. BMJ. 1990;300:899-902. (Level 4)

4. Hofman A, et al. JAMA. 1983;250:370-3. (Level 2)

5. Geleijnse JM, et al. Hypertension. 1997;29:913-7. (Level 2)

6. Parekh RS, et al. J Am Soc Nephrol. 2001;12:2418-26. (Level 4)

7. Van Dyck M, et al. Pediatr Nephrol. 1999;13:865-9. (Level 4)
CQ4 Are vaccinations recommended for children with CKD?

Children with CKD are especially susceptible to infectious diseases, and some of these diseases may become severe in these children. Therefore, we actively recommend that children with $\mathrm{CKD}$ receive vaccinations. (Grade C1)

Infectious diseases are serious factors that influence the prognosis of children with CKD. If children with $\mathrm{CKD}$ acquire an infectious disease, it has the potential to become severe, since children at advanced stages of CKD have low immunity, and some are also receiving immunosuppressive therapy. Vaccinations are effective preventive measures against infectious diseases, but it should be noted that vaccinations administered to children with $\mathrm{CKD}$ with low immunity may result in only low levels of antibody seroconversion, only mild antibody titer increase, and low persistence rates. There is also a possibility that a live vaccine could cause an infectious disease in the patient after the vaccination, and therefore, the use of live vaccines for children with CKD is often withheld.

There are two types of vaccines, inactivated and live, and each has advantages and disadvantages. Furthermore, the objective or effect of the vaccination differs depending on whether the child receiving it has received an adrenocorticosteroid, an immunosuppressant agent or no treatment at all. While caution is advised, if a disease is preventable by vaccination, it is even more important to vaccinate children with CKD than healthy children. Therefore, we actively recommend vaccinations for children with CKD. The seroconversion rate of antibody in children with CKD is reportedly slightly lower than in healthy children, but the effects of vaccinations on children with CKD are considered satisfactory.

Although inactivated vaccines of any kind are recommended, it has been reported that antibody titer levels are not maintained stably. Therefore, antibody titers should be checked several years after the vaccination and the patient should be re-vaccinated if necessary. If a child with nephrotic syndrome receives a dose of prednisolone (PSL) of $>2 \mathrm{mg} / \mathrm{kg}$ / day, vaccination is not recommended since seroconversion is unlikely. Live vaccines are recommended for children with CKD in general, but they are not recommended for children with CKD undergoing adrenocorticosteroid or immunosuppressive treatment. As a general rule, these patients should not be vaccinated until 3 months after terminating their immunosuppressive treatment. However, patients who are taking an immunosuppressant might be vaccinated if they reside in a region considered to be particularly high risk. 
For CKD in children undergoing adrenocorticosteroid therapy, vaccinations should be withheld until the dose of PSL is lower than $1 \mathrm{mg} / \mathrm{kg} /$ day or $2 \mathrm{mg} / \mathrm{kg} /$ every other day.

\section{Bibliography}

1. Prelog M, et al. Pediatr Transplant. 2007;11:73-6. (Level 4)

2. Broyer M, et al. Pediatrics. 1997;99:35-9. (Level 4)

3. Mori K, et al. Pediatr Int. 2009;51(5):617-20. (Level 4)

4. Mahmoodi M, et al. Eur Cytokine Netw. 2009;20:69-74. (Level 4)

5. Liakou CD, et al. Vaccine. 2011;29:6834-7. (Level 3)

6. Zamora I, et al. Pediatr Nephrol. 1994;8:190-2. (Level 4)

CQ5 Is antihypertensive drug therapy recommended for children with CKD to inhibit the progression of kidney dysfunction?

We recommend antihypertensive drug therapy for the treatment of children with CKD stage 2-4 because it inhibits the progression of renal insufficiency. (Grade B)

We suggest that renin-angiotensin system (RAS) inhibitors, including ACE inhibitors and ARBs be the first choice of agent for treating hypertension in children with proteinuric CKD. (Grade C1)

For children with CKD, we suggest a reduction in blood pressure to below the 90th percentile based upon the age, gender, and height of the patient as described by the NHBPEP. (Grade $\mathrm{C} 1$ )

Hypertension is one of the most common sequelae of children with CKD and it is prevalent only in the earlier stages of CKD. Hypertension is the highest risk factor for the progression of renal insufficiency and CVD.

\section{Antihypertensive drug therapy and children with CKD}

The ESCAPE Trial of 385 children with CKD (GFR between 15 and $80 \mathrm{~mL} / \mathrm{min}$ per $1.73 \mathrm{~m}^{2}$ ) reported that strict blood pressure (BP) control slows the progression of renal insufficiency and that the renoprotective effect of intensified BP control added to the potential benefit conferred by ACE inhibition. Therefore we recommend antihypertensive drug therapy for the treatment of children with CKD stage $2-4$ because it inhibits the progression of renal insufficiency.

\section{Antihypertensive agents for children with CKD}

Clinical studies have suggested that ACE inhibitors and ARBs are effective in reducing proteinuria and inhibiting the progression of CKD. Therefore we suggest that RAS inhibitors, including ACE inhibitors and ARBs, be the first choice for treating hypertension in children with proteinuric CKD. Calcium channel blockers are useful as addon therapy in children with resistant hypertension. The physician should select the antihypertensive agent according to the symptoms, because there is no conclusive evidence as to whether the inhibition of the renin-angiotensin system is superior to other antihypertensive agents in nonproteinuric CKD patients. In Japan, the ACE inhibitors, enalapril maleate and lisinopril, the ARB, valsartan, and the calcium antagonist, amlodipine besilate, are all approved as antihypertensive agents for use in children.

3. The definition of hypertension and target BP goals

The definition of hypertension in children is summarized in Table 16. The BP levels for children with CKD by age and height are shown in Table 17. For children with CKD, the National High Blood Pressure Education Program (NHBPEP) has recommended a reduction in BP to below the 90th percentile based upon the age, gender, and height of the patient (Table 17). BP in children with CKD should be more strictly controlled based on the findings of the ESCAPE Trial and the fact that hypertension is a risk factor for the progression of CKD and CVD. Correct measurement of BP in children requires the use of a cuff that is appropriate to the size of the child's upper right arm.

\section{Bibliography}

1. ESCAPE Trial Group, et al. N Engl J Med. 2009;361:1639-50. (Level 2)

2. Soergel M, et al. Pediatr Nephrol. 2000;15:113-8. (Level 4)

3. White CT, et al. Pediatr Nephrol. 2003;18:1038-48. (Level 3)

4. Franscini LM, et al. Am J Hypertens. 2002;15:1057-63. (Level 4)

5. von Vigier RO, et al. Eur J Pediatr. 2000;159:590-3. (Level 4)

6. Ellis D, et al. J Pediatr. 2003;143:89-97. (Level 4)

7. Ellis D, et al. Am J Hypertens. 2004;17:928-35. (Level 4)

8. Simonetti GD, et al. Pediatr Nephrol. 2006;21:1480-2 (Level 4)

Table 16 The definition of hypertension in children with CKD

\begin{tabular}{ll}
\hline Normal BP & $\begin{array}{c}\text { SBP and DBP that are }<90 \text { th percentile for gender, } \\
\text { age, and height }\end{array}$ \\
Prehypertension & $\begin{array}{c}\text { Average SBP or DBP levels that are } \geq 90 \text { th } \\
\text { percentile, but }<95 \text { th percentile for gender, age, } \\
\text { and height } \\
\text { Average SBP or DBP levels that are } \geq 120 / \\
80 \mathrm{mmHg}, \text { but }<95 \text { th percentile for gender, age, } \\
\text { and height } \\
\text { Average SBP and/or DBP that is } \geq 95 \text { th percentile } \\
\text { for gender, age, and height on at least } 3 \text { separate } \\
\text { occasions }\end{array}$ \\
\hline
\end{tabular}


Table 17 BP levels for boys and girls by age in the 50th percentile height

\begin{tabular}{|c|c|c|c|c|c|c|}
\hline \multirow[t]{2}{*}{ Age, years } & \multicolumn{3}{|c|}{ Boys SBP/DBP, mmHg } & \multicolumn{3}{|c|}{ Girls SBP/DBP, mmHg } \\
\hline & 90th & 95th & 99th & 90th & 95th & 99th \\
\hline 1 & $99 / 52$ & $103 / 56$ & $110 / 64$ & $100 / 54$ & $104 / 58$ & $111 / 65$ \\
\hline 2 & $102 / 57$ & $106 / 61$ & $113 / 69$ & $101 / 59$ & $105 / 63$ & $112 / 70$ \\
\hline 3 & $105 / 61$ & $109 / 65$ & $116 / 73$ & $103 / 63$ & $107 / 67$ & $114 / 74$ \\
\hline 4 & $107 / 65$ & $111 / 69$ & $118 / 77$ & $104 / 66$ & $108 / 70$ & $115 / 77$ \\
\hline 5 & $108 / 68$ & $112 / 72$ & $120 / 80$ & $106 / 68$ & $110 / 72$ & $117 / 79$ \\
\hline 6 & $110 / 70$ & $114 / 74$ & $121 / 82$ & $108 / 70$ & $111 / 74$ & $119 / 81$ \\
\hline 7 & $111 / 72$ & $115 / 76$ & $122 / 84$ & $109 / 71$ & $113 / 75$ & $120 / 82$ \\
\hline 8 & $112 / 73$ & $116 / 78$ & $123 / 86$ & $111 / 72$ & $115 / 76$ & $122 / 83$ \\
\hline 9 & $114 / 75$ & $118 / 79$ & $125 / 87$ & $113 / 73$ & $117 / 77$ & $124 / 84$ \\
\hline 10 & $115 / 75$ & $119 / 80$ & $127 / 88$ & $115 / 74$ & $119 / 78$ & $126 / 86$ \\
\hline 11 & $117 / 76$ & $121 / 80$ & $129 / 88$ & $117 / 75$ & $121 / 79$ & $128 / 87$ \\
\hline 12 & $120 / 76$ & $123 / 81$ & $131 / 89$ & $119 / 76$ & $123 / 80$ & $130 / 88$ \\
\hline 13 & $122 / 77$ & $126 / 81$ & $133 / 89$ & $121 / 77$ & $124 / 81$ & $132 / 89$ \\
\hline 14 & $125 / 78$ & $128 / 82$ & $136 / 90$ & $122 / 78$ & $126 / 82$ & $133 / 90$ \\
\hline 15 & $127 / 79$ & $131 / 83$ & 138/91 & $123 / 79$ & $127 / 83$ & $134 / 91$ \\
\hline 16 & $130 / 80$ & $134 / 84$ & $141 / 92$ & $124 / 80$ & $128 / 84$ & $135 / 91$ \\
\hline 17 & $132 / 82$ & $136 / 87$ & $143 / 94$ & $125 / 80$ & $129 / 84$ & $136 / 91$ \\
\hline
\end{tabular}

Falkner B, et al. Pediatrics. 2004;114:555-76

CQ6 Does the use of RAS inhibitors for the treatment of children with CKD inhibit the progression of renal insufficiency?

We recommend using ACE inhibitors for the treatment of children with CKD with hypertension or proteinuria because they appear to slow the progression of renal insufficiency. (Grade B)(not covered by Japanese health insurance)

We consider it to be acceptable to use ARBs for the treatment of children with CKD with hypertension or proteinuria because they have the potential to inhibit the progression of renal insufficiency. (Grade C1) (not covered by the Japanese health insurance)

We do not recommend combination therapy with ACE inhibitors and ARBs for the treatment of children with CKD with hypertension or proteinuria because there is no conclusive evidence that this combination therapy can inhibit the progression of renal insufficiency. (Grade C2)

It is not known if RAS inhibitors administered for the treatment of CKD in children without hypertension or proteinuria can inhibit the progression of renal insufficiency.
1. Use of ACE inhibitors for children with CKD

Retrospective studies have suggested ACE inhibitors decrease proteinuria and slow the progression of renal insufficiency. The ESCAPE Trial reported that strict blood pressure control using ramipril slowed the progression of renal insufficiency. However, the ACE inhibitors are not approved as renoprotective agents. The dose of ACE inhibitors (enalapril and ramipril) approved as antihypertensive agents for children in Japan should serve as the reference dose.

\section{Use of ARBs for children with CKD}

Retrospective studies have suggested that ARBs decrease proteinuria and inhibit the progression of renal insufficiency. A double-blind multinational study of 306 children with CKD reported that losartan significantly lowered proteinuria and was well tolerated after 12 weeks in children with proteinuria with or without hypertension. ARBs are not approved as renoprotective agents. The dose of ARBs (valsartan) approved as antihypertensive agents of children in Japan should serve as the reference dose.

3. Combination therapy with ACE inhibitors and ARBs for children with CKD

The efficacy of combination therapy with ACE inhibitors and ARBs compared with single agent therapy (ACE inhibitor or ARB) has not been investigated in any RCTs. Therefore, we cannot recommend combination therapy for the treatment of children with CKD with hypertension or proteinuria. Both ACE inhibitors and ARBs should be used cautiously if the GFR is less than $60 \mathrm{~mL} / \mathrm{min}$ per $1.73 \mathrm{~m}^{2}$. Since the decline in GFR and hyperkalemia induced by RAS inhibition typically occurs within the first few days after the onset of therapy, the serum creatinine and potassium concentrations should be monitored.

\section{Bibliography}

1. Soergel M, et al. Pediatr Nephrol. 2000;15:113-8. (Level 4)

2. Wühl E, et al. Kidney Int. 2004;66:768-76. (Level 4)

3. Ardissino G, et al. Nephrol Dial Transplant. 2007;22:2525-30. (Level 4)

4. ESCAPE Trial Group, et al. N Engl J Med. 2009;361:1639-50. (Level 2)

5. von Vigier RO, et al. Eur J Pediatr. 2000;159:590-3. (Level 4)

6. Ellis D, et al. J Pediatr. 2003;143:89-97. (Level 4)

7. Ellis D, et al. Am J Hypertens. 2004;17:928-35. (Level 4)

8. Simonetti GD, et al. Pediatr Nephrol. 2006;21:1480-2. (Level 4)

9. Franscini LM, et al. Am J Hypertens. 2002;15:1057-63. (Level 4)

10. White CT, et al. Pediatr Nephrol. 2003;18:1038-43. (Level 3)

11. Webb NJ, et al. Clin J Am Soc Nephrol. 2010;5:417-24. (Level 2)

12. Seeman T, et al. Kidney Blood Press Res. 2009;32:440-4. (Level 4)

13. Litwin M, et al. Pediatr Nephrol. 2006;21(11):1716-22. (Level 4) 
CQ7 Is management of CKD-MBD (chronic kidney disease-mineral and bone disorders) recommended to improve growth and the survival rate of children with CKD?

We recommend an appropriate management of CKDMBD to prevent growth retardation and CVD. Prevention of CVD may lead to an improvement in the survival rate of children with CKD. (Grade B)

We recommend that the target serum calcium $(\mathrm{Ca})$ and phosphorus $(\mathrm{P})$ levels be within the normal range for the patient's age at all CKD stages. (Grade C1) We recommend that the target serum $\mathrm{Ca} \times \mathrm{P}$ product be $<65 \mathrm{mg}^{2} / \mathrm{dL}^{2}$ in children aged less than 12 years and $<55 \mathrm{mg}^{2} / \mathrm{dL}^{2}$ in children aged 12 years or older at CKD stages 3-5. (Grade C1)

We recommend that the target serum intact PTH level be within the normal range at CKD stages $2-3,<100 \mathrm{pg} / \mathrm{mL}$ at stage 4 , and $100-300 \mathrm{pg} / \mathrm{mL}$ at stages 5 and 5D. (Grade C1)

It has been shown that CKD-MBD causes not only bone disorders, but also cardiovascular calcification, which may have a direct impact on survival. In children who are still growing, CKD-MBD also causes bone pain, limb deformities, bone fracture, and growth retardation, which impair the patient's quality of life. This CQ is aimed at determining whether appropriate management of parameters in CKD-MBD, such as serum calcium, phosphorus, and parathyroid hormone (PTH), improves growth and prevents CVD in children with CKD.

An observational study employing bone biopsies performed on 55 children undergoing peritoneal dialysis (PD) showed that higher levels of PTH were significantly associated with high-turnover lesions in the bone and lower levels of PTH with adynamic bone. In addition, an international survey of 890 children undergoing PD showed that higher PTH levels were significantly associated with osteopenia, bone pain, limb deformities, growth retardation, and extraosseous calcifications. Therefore, serum PTH should be appropriately managed to prevent bone disorder and growth retardation. In regard to the prevention of CVD, studies in children and young adults showed that cardiac calcification was associated with serum $\mathrm{PTH}$, phosphorus, and $\mathrm{Ca} \times \mathrm{P}$ product. Other reports showed that carotid intima-media thickness correlated with $\mathrm{Ca} \times \mathrm{P}$ and that left ventricular hypertrophy and poor diastolic function correlated with higher levels of PTH in children with CKD. Therefore, CKD-MBD should be appropriately managed to prevent CVD.

The recommendations regarding the target levels of serum calcium, phosphorus, $\mathrm{PTH}$, and $\mathrm{Ca} \times \mathrm{P}$ product are based on observational studies and international guidelines including the KDOQI guidelines, KDIGO guidelines, and European Pediatric Dialysis Working Group guidelines.

In summary, CKD-MBD should be managed appropriately to prevent growth retardation, bone disorder, and CVD.

\section{Bibliography}

1. Seikaly MG, et al. Pediatr Nephrol. 2006;21:793-9. (Level 4)

2. Waller SC, et al. Kidney Int. 2005;67:2338-45. (Level 4)

3. Waller S, et al. Pediatr Nephrol. 2003;18:1236-41. (Level 4)

4. Salusky IB, et al. Kidney Int. 1994;45:253-8. (Level 4)

5. Borzych D, et al. Kidney Int. 2010;78:1295-304. (Level 4)

6. Civilibal M, et al. Pediatr Nephrol. 2006;21:1426-33. (Level 4)

7. Shroff RC, et al. J Am Soc Nephrol. 2007;18:2996-3003. (Level 4)

8. Goodman WG, et al. N Engl J Med. 2000;342:1478-83. (Level 4)

9. Lumpaopong A, et al. Transplant Proc. 2007;39:37-9. (Level 4)

10. Oh J, et al. Circulation. 2002;106:100-5. (Level 4)

11. Milliner DS, et al. Kidney Int. 1990;38:931-6. (Level 4)

12. Civilibal M, et al. Pediatr Nephrol. 2009;24:555-63. (Level 4)

13. Litwin M, et al. J Am Soc Nephrol. 2005;16:1494-500. (Level 4)

14. Mitsnefes MM, et al. J Am Soc Nephrol. 2005;16:2796-803. (Level 4)

15. Salusky IB, et al. J Am Soc Nephrol. 2005;16:2501-8. (Level 2)

16. Pieper AK, et al. Am J Kidney Dis. 2006;47:625-35. (Level 2)

17. Gulati A, et al. Int Urol Nephrol. 2010;42:1055-62. (Level 2)

CQ8 Is it recommended that the concentration of hemoglobin be maintained above $11 \mathrm{~g} / \mathrm{dL}$ for the treatment of anemia in order to improve the prognosis and survival of children with CKD?

We suggest maintaining $\mathrm{Hb}$ values above $11 \mathrm{~g} / \mathrm{dL}$ to improve the prognosis and survival of children with CKD. (Grade C1)

However, the upper limit of $\mathrm{Hb}$ values is not set.

The effects of renal anemia on the glomerular filtration rate in children have not been clarified. Whether uncontrolled anemia in children with CKD affects their prognosis and what the normal $\mathrm{Hb}$ levels are in children with $\mathrm{CKD}$ also remain unclear. Anemia in children with CKD strongly affects the cardiovascular system as well as the kidneys. In particular, it causes left ventricular failure, leading to prolonged hospital stays, and eventually to a higher mortality rate. It has been reported that early therapeutic intervention contributes to a child's growth and improves IQ and QOL. Therefore, treatment should be administered if the patient has been diagnosed with anemia. Treatment should continue until the $\mathrm{Hb}$ value exceeds 
Table 18 Normal Hb values for children(g/dL)

\begin{tabular}{|c|c|c|c|c|c|c|}
\hline & \multicolumn{3}{|l|}{ Boys } & \multicolumn{3}{|l|}{ Girls } \\
\hline & Mean & SD & $\begin{array}{l}<5 \text { th } \\
\text { percentile }\end{array}$ & Mean & SD & $\begin{array}{l}<5 \text { th } \\
\text { percentile }\end{array}$ \\
\hline 1 year $<$ & 14.7 & 1.4 & 12.1 & 13.2 & 1.1 & 11.4 \\
\hline $1-2$ years & 12.0 & 0.8 & 10.7 & 12.0 & 0.8 & 10.8 \\
\hline $3-5$ years & 12.4 & 0.8 & 11.2 & 12.4 & 0.8 & 11.1 \\
\hline $6-8$ years & 12.9 & 0.8 & 11.5 & 12.8 & 0.8 & 11.5 \\
\hline $9-11$ years & 13.3 & 0.8 & 12.0 & 13.1 & 0.8 & 11.9 \\
\hline $12-14$ years & 14.1 & 1.1 & 12.4 & 13.3 & 1.0 & 11.7 \\
\hline $15-19$ years & 15.1 & 1.0 & 13.5 & 13.2 & 1.0 & 11.5 \\
\hline
\end{tabular}

NHANESIIIdata, United States, 1988-1994

Table 19 Normal $\mathrm{Hb}$ values for infants(g/dL)

Nathan and Oski's Hematology of Infancy and Childhood (ed 6)

\begin{tabular}{lll}
\hline & Mean & -2 SD* \\
\hline Term & 16.5 & 13.5 \\
1-3 days & 18.5 & 14.5 \\
1 week & 17.5 & 13.5 \\
2 weeks & 16.5 & 12.5 \\
1 month & 14.0 & 10.0 \\
2 months & 11.5 & 9.0 \\
3-6 months & 11.5 & 9.5 \\
6-24 months & 12.0 & 10.5 \\
\hline
\end{tabular}

$11 \mathrm{~g} / \mathrm{dL}$. Note, however, that although the upper limit of the $\mathrm{Hb}$ value in children has not yet been set, $\mathrm{Hb}$ values in adults are defined such that one should not intentionally exceed $13 \mathrm{~g} / \mathrm{dL}$. In addition, adequate attention should also be paid to such problems as hypertension and vascular access troubles in the treatment of anemia in children with CKD (Tables 18, 19).

\section{Bibliography}

1. Filler G, et al. Pediatr Nephrol. 2007;22:702-7. (Level 4)

2. Singh AK, et al. N Engl J Med. 2006;355:2085-98. (Level 2)

3. Pfeffer MA, et al. N Engl J Med. 2009;361:2019-32. (Level 2)

4. Jabs K. Pediatr Nephrol. 1996;10:324-7. (Level 2)

5. Warady BA, et al. Pediatr Nephrol. 2003;18:1055-62. (Level 4)

6. Warady BA, et al. Pediatr Nephrol. 2006;21:1144-52. (Level 2)

CQ9 Is treatment of growth retardation

with recombinant human growth hormone (rhGH) recommended for children with CKD?

We recommend treatment with rhGH for prepubescent children with CKD at stages 3-5 with accompanying growth retardation. (Grade B)
Growth impairment is one of the major visible complications of CKD in children. Currently, rhGH is used to treat growth impairment in children with CKD and is covered by health insurance in Japan. A concern is whether rhGH therapy should be administered to all children with CKD who have growth impairment.

Various randomized controlled trials reported that adequate growth in stature was obtained in 2 or 3 years after the start of rhGH treatment. We recommend administering rhGH at $28 \mathrm{IU} / \mathrm{m}^{2} /$ week (or approximately $0.35 \mathrm{mg} / \mathrm{kg} /$ week). In infants, it is preferable to first consider appropriate nutritional intake, and then consider rhGH therapy as well.

\section{Bibliography}

1. Seikaly MG, et al. Pediatr Nephrol. 2009;24:1711-7. (Level 4)

2. Muller-Wiefel D, et al. Clin Nephrol. 2010;74:97-105. (Level 4)

3. Berard E, et al. Pediatr Nephrol. 2008;23:2031-8. (Level 4)

4. Vidal E, et al. Nephrol Dial Transplant. 2012;27:388-95. (Level 4)

5. Kari JA, et al. Kidney Int. 2000;57:1681-7. (Level 4)

6. Mencarelli F, et al. Pediatr Nephrol. 2009;24:1039-46. (Level 4)

7. Pape L, et al. Transplant Proc. 2006;38:685-7. (Level 4)

8. Fine RN, et al. Kidney Int. 2002;62:688-96. (Level 2)

9. Fine RN, et al. Pediatr Nephrol. 2010;25:739-46. (Level 4)

10. Nissel R, et al. Microvasc Res. 2009;78:246-52. (Level 4)

11. Dharnidharka VR, et al. Pediatr Transplant. 2008;12:689-95. (Level 4)

CQ10 Is urological intervention for urinary tract system abnormalities in children with CKD recommended to prevent the progression of renal dysfunction?

For children with CKD, we recommend carefully evaluating urinary tract system abnormalities and considering urological intervention for preventing the possible progression of renal dysfunction. (Grade C1)

The most common condition responsible for children with CKD is congenital anomalies of the kidney and urinary tract (CAKUT). Structural anomalies in the CAKUT spectrum are most commonly renal dysplasia and hypoplasia, often accompanied by anomalies of the extrarenal urinary tract system. Typical disorders include vesicoureteric reflux (VUR), obstructive urinary tract disorders [e.g. hydronephrosis, posterior urethral valves (PUV)], and bladder dysfunction.

For all children with CKD resulting from CAKUT, it is recommended that the history of the child's voiding patterns be taken and that an ultrasonography be taken of the whole urinary tract. If obstruction of the urinary tract is 
suggested or abnormal bladder morphology is present, various imaging modalities, urodynamic testing, endoscopy, and other tests should be considered for further evaluation. In all patients determined to require a renal transplant, a voiding cystourethrogram (VCUG) is recommended to identify any VUR and evaluate the bladder and the urethral morphology and function. Patients with urinary system abnormalities that are confirmed as a result of these examinations require appropriate intervention.

\section{Management of VUR in children with CKD}

For VUR in children with CKD, further studies are necessary to elucidate whether prophylactic antimicrobial therapy or antireflux surgery can improve renal prognosis. VUR can be secondary to lower urinary tract abnormalities or other abnormalities, and those primary abnormalities require attention.

2. Management of lower urinary tract abnormalities in children with CKD

Among lower urinary tract abnormalities, particularly severe conditions are bladder dysfunction, PUV, and other urethral obstructive diseases. Bladder dysfunction can result not only from a neurological abnormality (neurogenic bladder), but also from other disorders without apparent neurological abnormalities, such as Hinman's syndrome, urethral abnormalities including PUV, and polyuria associated with impaired urinary concentration. It is important to carefully take the history of the child's voiding patterns (e.g., number of voidings per day, time of voiding, urgency, daytime enuresis, nocturnal enuresis) and any constipation, and perform ultrasonography to evaluate bladder morphology and wall thickening. If ultrasonography suggests an abnormality, the urodynamics should be evaluated. In patients with confirmed bladder dysfunction, the prognosis of renal function may be improved by clean intermittent catheterizations (CIC), anticholinergic medication, or surgical bladder augmentation.

3. Management of urinary tract abnormalities in renal transplant recipients

Management of urinary tract abnormalities is an important factor for successful maintenance of renal function after renal transplantation. In post-renal transplant patients, it has been suggested that VUR causes not only pyelonephritis, but also impaired function of the transplanted kidney. On the other hand, with appropriate diagnosis and urological intervention before renal transplantation in such patients, the prognosis for the transplanted kidney's function has been shown to be comparable to that in patients without a lower urinary tract disorder.

\section{Bibliography}

1. Ishikura K, et al. Nephrol Dial Transplant. 2013 (Epub ahead of print). (Level 4)

2. Hattori S, et al. Pediatr Nephrol. 2002;17:456-61. (Level 5)

3. Ardissino G, et al. Pediatrics. 2003;111:e382-e387. (Level 4)

4. DeFoor W, et al. J Urol. 2008;180:1705-8. (Level 4)

5. Neuhaus TJ, et al. J Urol. 1997;157:1400-3. (Level 4)

6. Adams J, et al. Transpl Int. 2004;17:596-602. (Level 4)

7. Irtan S, et al. Pediatr Transplant. 2010;14:512-9. (Level 4)

8. Aki FT, et al. Transplant Proc. 2006;38:554-5. (Level 5)

9. Nahas WC, et al. J Urol. 2008;179:712-6. (Level 4)

10. Mendizabal S, et al. J Urol. 2005;173:226-9 (Level 4)

CQ11 What is recommendation regarding renal replacement therapy (RRT) as a first line treatment for CKD in children?

We suggest renal transplantation as a first line treatment for RRT since there is some evidence that it improves the prognoses for children with CKD. (Grade C1)

For children with CKD on a transplant waiting list, we recommend peritoneal dialysis as the renal replacement therapy until the patient receives the renal transplant. (Grade B)

Renal replacement therapy is considered for CKD stages 4 and 5 . In children as well as adults, hemodialysis, peritoneal dialysis, and renal transplantation are among the top therapies of choice. The question is which therapy is optimal for CKD in children who must grow physically, mentally, and socially, including in their infancy when performing RRT is technically difficult, as well as in puberty when drug compliance and other issues arise.

When simply comparing survival rates, renal transplantation is the best treatment for RRT. Even though the patient must undergo temporary dialysis, renal transplantation is the ultimate choice from the viewpoint of both the patient's prognosis and QOL. If a child with CKD is treated with chronic dialysis, peritoneal dialysis is preferable, considering the techniques and the QOL (including growth and development, as well as acquisition of social abilities). Whichever renal replacement therapy is chosen, it is important to provide information to the patient's parents and consult with them from a long-term perspective. Coordinated care involving mental, social and physical aspects is especially necessary for children and adolescents. Such care should involve not only doctors, but also nurses, psychotherapists, dietitians, social workers, teachers, and other appropriate professionals. 


\section{Bibliography}

1. McDonald SP, et al. N Engl J Med. 2004;26:2654-62. (Level 4)

2. Butani L, et al. Transplantation. 2011;91:447-51. (Level 4)

3. Sinha R, et al. Pediatr Transplant. 2010;14:583-8. (Level 4)

4. Nishikawa K, et al. Clin Transpl. 2002;367-77. (Level 4)

5. Chung AW, et al. Nephrol Dial Transplant. 2010;25:4031-41. (Level 4)

6. Buyan N, et al. Pediatr Nephrol. 2010;25:1487-96. (Level 4)

7. Pape L, et al. Transplant Proc. 2006;38:685-7. (Level 4)

8. Icard P, et al. Pediatr Transplant. 2010;14:887-90. (Level 4)

9. Shroff R, et al. Pediatr Nephrol. 2009;24:463-74. (Level 4)

10. Yata N, et al. Pediatr Nephrol. 2004;19:1062-64. (Level 5)

11. Tyden G, et al. Pediatr Transplant. 2011;15:502-4. (Level 4)

12. Kennedy SE, et al. Transplantation. 2006;82:1046-50. (Level 4)

\section{Chapter 18: Initiation of dialysis}

CQ1 When should general physicians refer their CKD patients to specialists in order to delay the timing for renal replacement therapies?

We recommend that general physicians refer their patients to specialists when the CKD stage has increased to $\mathrm{G} 3$ or greater as referral can delay the course of renal disease and the timing for renal replacement therapies.

It has been reported that the risk of cardiovascular events and the rate of worsening of renal function significantly increased in CKD patients when their eGFR was reduced to less than $50 \mathrm{ml} / \mathrm{min} / 1.73 \mathrm{~m}^{2}$. Therefore, early referral of such patients to nephrologists is usually recommended. However suitable timing of the referral remains uncertain. To our knowledge, no prospective studies have been conducted to directly address this question. Some small, retrospective or uncontrolled studies indicated that early referral at CKD stage G3 or greater can slow down the course of renal disease, which may consequently delay the timing for renal replacement therapies and ultimately, related mortality. Other retrospective studies also have indicated that early referral has some advantages after the initiation of renal replacement therapies, such as decreasing complications and improving survival. There have been some studies demonstrating that early treatment at a nephrology clinic with a multidisciplinary team (such as a pharmacy specialist, a diabetes educator, a dietitian, a social worker, and a nephrology nurse) may slow the decline in the patient's renal function. Additional prospective studies are needed to establish the usefulness of early referral in delaying the timing of renal replacement therapies.
Bibliography

1. Black C, et al. Health Technol Assess. 2010;14:1-184. (Level 4)

2. Orlando LA, et al. N C Med J. 2007;68:9-16. (Level 4)

3. Nakamura S, et al. Circ J. 2007;71:511-6. (Level 4)

4. Chen SC, et al. Nephrology (Carlton). 2008;13:730-6. (Level 4)

5. Jones C, et al. Nephrol Dial Transplant. 2006;21:2133-43. (Level 4)

6. Martinez-Ramirez HR, et al. Am J Kidney Dis. 2006;47:78-87. (Level 3)

7. Jungers P, et al. Nephrol Dial Transplant. 2001;16:2357-64. (Level 4)

8. Bayliss EA, et al. Clin J Am Soc Nephrol. 2011;6:704-10. (Level 4)

9. Barrett BJ, et al. Clin J Am Soc Nephrol. 2011;6:1241-7. (Level 2)

10. Kessler M, et al. Am J Kidney Dis. 2003;42:474-85. (Level 4)

11. Kinchen KS, et al. Ann Intern Med. 2002;137:479-86. (Level 4)

12. Roderick P, et al. Nephrol Dial Transplant. 2002;17:1252-9. (Level 4)

CQ2 What are the criteria for initiating dialysis to improve the survival of patients with CKD?

Early initiation of dialysis in patients with no symptoms of uremia and an eGFR of $8-14 \mathrm{ml} / \mathrm{min} / 1.73 \mathrm{~m}^{2}$ does not contribute to a better prognosis. However, initiation before reaching an eGFR of $2 \mathrm{ml} / \mathrm{min} /$ $1.73 \mathrm{~m}^{2}$ is desirable even when the patient is asymptomatic.

In the past, early initiation of dialysis was suggested as a means to improve survival, and there was a tendency to start dialysis even though the eGFR was relatively high. However, in recent years, there have been several negative reports on the early initiation of dialysis and better survival after later dialysis initiation. The negative results of the IDEAL study, which was an RCT that compared early with late initiation, were not cited in the CKD clinical practice guidelines 2009 in Japan.

Consensus among various related societies in Japan and several overseas guidelines have suggested that the initiation of dialysis is required in patients with progressive renal dysfunction with an eGFR value of $<15 \mathrm{ml} / \mathrm{min} / 1.73 \mathrm{~m}^{2}$ and clear positive symptoms of uremia. According to recent observational studies (e.g. ERA-EDTA registry, USRDS registry), patients who were initiated on dialysis at an eGFR value of approximately $5-10 \mathrm{ml} / \mathrm{min} / 1.73 \mathrm{~m}^{2}$ showed a significantly better survival, compared with those who were initiated at a value of less than 5 or more than $10 \mathrm{ml} / \mathrm{min} / 1.73 \mathrm{~m}^{2}$. In addition, an analysis of Japanese patients enrolled in the JSDT registry showed that initiation at an eGFR value of $<8 \mathrm{ml} / \mathrm{min} /$ $1.73 \mathrm{~m}^{2}$ was associated with a better prognosis and initiation at an eGFR value of $<2 \mathrm{ml} / \mathrm{min} / 1.73 \mathrm{~m}^{2}$ was associated with a poorer prognosis. The results of the IDEAL study, the only RCT on this topic, were published in 2010. In this study, a comparison of survival between an early initiation group (eGFR of $10-14 \mathrm{ml} / \mathrm{min} / 1.73 \mathrm{~m}^{2}$ ) and a late initiation group (5-7 ml/ 
$\min / 1.73 \mathrm{~m}^{2}$ ) was conducted. However, better results for allcause mortality were not obtained in the early initiation group.

\section{Bibliography}

1. Stel VS, et al. Nephrol Dial Transplant. 2009;24:3175-82. (Level 4)

2. Wright S, et al. Clin J Am Soc Nephrol. 2010;5:1828-35. (Level 4)

3. Cooper BA, et al. N Engl J Med. 2010;363:609-19. (Level 2)

4. Yamagata K, et al. Ther Apher Dial. 2012;16:54-62. (Level 4)

5. Wagner M, et al. Am J Kidney Dis. 2011;57:894-902. (Level 4)

6. Couchoud C, et al. Nephrol Dial Transplant. 2009;24:1553-61. (Level 4)

7. Portoles J, et al. Perit Dial Int. 2009;29:150-7. (Level 4)

8. Shafi T, et al. Am J Kidney Dis. 2010;56:348-58. (Level 4)

9. Yamagata K, et al. Ther Apher Dial. 2012;16:111-120. (Level 4)

CQ3 When is permanent vascular access recommended to be provided in order to improve survival after the initiation of hemodialysis

We recommend avoidance of initiation of hemodialysis with a central vein catheter (CVC) as vascular access, because initiation with a CVC could be related to a poorer survival. (Grade $\mathrm{C} 2$ )

We suggest creation of an arteriovenous fistula or a graft preferably at 30 days or at least 14 days before the estimated timing of the first puncture. (Grade $\mathrm{C} 1$ )

Vascular access with a CVC has reportedly been associated with a poor prognosis. Therefore, initiation of dialysis with a CVC should be avoided as much as possible. On the other hand, occlusion or failure of an arteriovenous fistula (AVF) or a graft (AVG) subsequently leads to the use of a CVC. It has been reported that the timing of the first puncture after placement of an AVF or AVG was associated with the patency of access. Therefore, we deduced that the timing of the first cannulation at a time when access patency is maximized can influence survival.

Several studies have investigated the relationship between the timing of the first puncture and subsequent access patency. Their results have shown that patients who started their hemodialysis treatment either within 14 or 30 days after the creation of an AVF experienced a higher incidence of access failure. Moreover, a beneficial effect of AVF or AVG at the initiation of hemodialysis was also demonstrated from an economic view point; the population of patients with functioning access at the start of hemodialysis treatment incurred a lower health care cost. Facilitybased analysis conducted by DOPPS demonstrated that the characteristics of blood access rather than practice pattern of each facility affected access failure. Therefore, we recommend the creation of an AVF or AVG at least 14-30 days before the initiation of hemodialysis.

\section{Bibliography}

1. Lorenzo V, et al. Am J Kidney Dis. 2004;43:999-1007. (Level 4)

2. Wasse H, et al. Sem Dial. 2008;21:483-9. (Level 4)

3. Ng LJ, et al. Nephrol Dial Transplant. 2011;26:3659-66. (Level 4)

4. Rayner HC, et al. Kidney Int. 2003;63:323-30. (Level 4)

5. Ravani P, et al. J Am Soc Nephrol. 2004;15:204-9. (Level 4)

6. Wu LC, et al. Kaohsiung J Med Sci. 2009;25:521-9. (Level 4)

7. Saran R, et al. Nephrol Dial Transplant. 2004;19:2334-40. (Level 4)

\section{Chapter 19: Kidney transplantation}

CQ1 Is preemptive kidney transplantation recommended to improve mortality?

We recommend preemptive kidney transplantation to improve mortality compared to kidney transplantation after the institution of dialysis.

Preemptive kidney transplantation (PEKT) has been shown to improve mortality and graft survival and to decrease rejection rates. It has also been suggested that PEKT may improve the quality of life by allowing the patient to be free from dialysis and related drawbacks such as a strict diet, fluid control, and hospitalization due to vascular access trouble. These advantages may be even greater for pediatric patients in order to support healthy growth. Medical financial issues also favor PEKT compared to the institution of dialysis. Recent studies have, however, suggested that PEKT may only have a favorable outcome compared with cadaveric kidney transplantation. It is anticipated that further studies will clarify the advantages of PEKT compared with more recent data on kidney survival in non-PEKT patients.

Timing of PEKT should be judged carefully since performing PEKT too early, before reaching the eGFR value of $15 \mathrm{ml} / \mathrm{min} / 1.73 \mathrm{~m}^{2}$, has not shown benefit in terms of mortality compared to an eGFR value below $15 \mathrm{ml} / \mathrm{min} /$ $1.73 \mathrm{~m}^{2}$. However, attempting PEKT with optimal timing is recommended as it requires careful monitoring and control, which is expected to lead to comprehensive management of the CKD patient.

\section{Bibliography}

1. Mange KC, et al. N Engl J Med. 2001;344:726-31. (Level 4)

2. Vats AN, et al. Transplantation. 2000;69:1414-9. (Level 4)

3. Mange KC, et al. Nephrol Dial Transplant. 2003;18:172-7. (Level 4)

4. Meier-Kriesche HU, et al. Kidney Int. 2000;58:1311-7. (Level 4)

5. Meier-Kriesche HU, et al. Transplantation. 2002;74:1377-81. (Level 4) 
6. Kasiske BL, et al. J Am Soc Nephrol. 2002;13:1358-64. (Level 4)

7. Harada H, et al. Int J Urol. 2001;8:205-11. (Level 4)

8. Jung GO, et al. Transplantation Proc. 2010;42:766-74. (Level 4)

9. Witczak BJ, et al. Transplantation. 2009;88:672-7. (Level 4)

10. Cransberg K, et al. Am J Transplant. 2006;6:1858-64. (Level 4)

11. Ishani A, et al. Am J Kidney Dis. 2003;42:1275-82. (Level 4)

12. Akkina SK, et al. Am J Transplant. 2008;8:2071-6. (Level 4)

CQ2 What are the strategies for pre-transplant CKD management to improve mortality and kidney survival in kidney transplant patients?

To improve mortality and kidney survival in kidney transplant patients, we recommend careful prevention and management of CKD and related complications (anemia, CKD-MBD, CVD, metabolic syndrome, infection etc.).

The innovative development of immunosuppressants has led to lower rates of rejection and better kidney survival. This has generated new strategies to improve survival with a functioning graft. Recommendations related to pretransplant CKD management for better survival after transplantation are mentioned below.

Anemia in CKD

Anemia in CKD patients should be controlled (see chapter 7) before transplantation.

CKD-MBD

CKD-MBD in CKD patients (see chapter 8) should be controlled before transplantation.

Cardiovascular disease

Cardiovascular disease (CVD) in CKD patients (see chapter 4) should be evaluated and aggressively treated before transplantation.

Obesity and metabolic syndrome

Obesity in CKD patients (see chapter 15) should be evaluated and treated before transplantation.

Smoking

Quitting smoking before transplantation is recommended. Infection

Aggressive immunization with vaccines should be started from the early stage of CKD.

Recurrence of primary kidney disease

Effort should be made to clarify the primary disease that led to end-stage renal disease and determine the relationship, at the time of transplantation, with the possibility of disease recurrence.

\section{Bibliography}

1. Wolfe RA, et al. N Engl J Med. 1999;341:1725-30. (Level 4)
2. Tojimbara T, et al. Am J Transplant. 2007;7:609-17. (Level 4)

3. Djamali A, et al. Transplantation. 2003;76:816-20. (Level 4)

4. Campise M, et al. Nephrol Dial Transplant. 2005;20(suppl 8):viii8-viii12. (Level 4)

5. Choukroun G, et al. J Am Soc Nephrol. 2012;23:360-8. (Level 2)

6. Ball AM, et al. JAMA. 2002;288:3014-8. (Level 4)

7. Vautour LM, et al. Osteoporos Int. 2004;15:160-7. (Level 4)

8. Kanaan N, et al. Clin J Am Soc Nephrol. 2010;5:1887-92. (Level 4)

9. Messa P, et al. Kidney Int. 1998;54:1704-13. (Level 4)

10. Kawarazaki H, et al. Nephrol Dial Transplant. 2011;26:2691-5. (Level 4)

11. Go AS, et al. N Engl J Med. 2004;351:1296-305. (Level 4)

12. Meier-Kriesche HU, et al. Am J Transplant. 2004;4:1662-8. (Level 4)

13. Jones DG, et al. Am J Transplant. 2009;9:1846-52. (Level 4)

14. De Lima JJ, et al. Transplantation. 2010;89:845-50. (Level 4)

15. Patel RK, et al. Clin J Am Soc Nephrol. 2008;3:1807-11. (Level 4)

16. McGregor E, et al. Nephrol Dial Transplant. 2000;15:93-8. (Level 5)

17. Levin A, et al. Am J Kidney Dis. 1999;34:125-34. (Level 4)

18. Chen SC, et al. Clin J Am Soc Nephrol. 2011;6:2750-8. (Level 4)

19. Foley RN, et al. Clin J Am Soc Nephrol. 2010;5:805-13. (Level 2)

20. Johnson DW, et al. Transplantation. 2002;74:675-81. (Level 4)

21. Kasiske BL, et al. Am J Transplant. 2003;3:178-85. (Level 4)

22. Molnar MZ, et al. Kidney Int. 2011;80:218-24. (Level 4)

23. Cacciola RA, et al. Transplant Proc. 2008;40:3408-12. (Level 4)

24. Kovesdy CP, et al. Am J Transplant 2010;10:2644-51. (Level 4)

25. Nogueira JM, et al. Am J Kidney Dis. 2010;55:907-15. (Level 4)

26. Fabrizi F, et al. Am J Transplant. 2005;5:2913-21. (Level 1)

27. Reddy PN, et al. Clin J Am Soc Nephrol. 2011;6:1481-7. (Level 4)

28. Burdick RA, et al. Kidney Int. 2003;63:2222-9. (Level 5)

29. Harnett JD, et al. Transplantation. 1987;44:369-76. (Level 5)

30. DaRoza G, et al. Am J Kidney Dis. 2003;42:1184-92. (Level 4)

31. Mathurin P, et al. Hepatology. 1999;29:257-63. (Level 4)

32. Werner T, et al. Transplantation. 2010;90:407-11. (Level 4)

33. Bloom RD, et al. Am J Transplant. 2005;5:139-44. (Level 4)

34. Torre-Cisneros J, et al. Clin Infect Dis. 2009;48:1657-65. (Level 4)

35. Chailimpamontree W, et al. J Am Soc Nephrol. 2009;20:843-51. (Level 4)

36. Briganti EM, et al. N Engl J Med. 2002;347:103-9. (Level 4)

37. Little MA, et al. Nephrol Dial Transplant. 2009;24:3219-25. (Level 4)

CQ3 What are the strategies to preserve kidney function and mortality in living kidney donors?

We recommend adequate evaluation of kidney function and elimination or treatment of factors that may retard kidney function before transplantation.

We recommend monitoring for the occurrence of hypertension, albuminuria/hematuria and other risk factors of CVD after kidney donation as in general CKD management. 
Most Japanese donors, especially the elderly, develop CKD stage 3 after kidney donation. Evidence related to kidney function and survival in such donors after transplantation has accumulated and is hereby reviewed for adequate management.

Mortality

Adequate evaluation of donors before the transplantation leads to a better survival.

Kidney survival

Kidney survival in adequately evaluated donors before transplantation leads to a good prognosis. However, kidney function should be surveyed over the long-term.

Hypertension and CVD

Complications in adequately evaluated donors before transplantation do not exacerbate, but the incidence of hypertension may increase and should, therefore, be carefully monitored over the long-term.

Quality of life

Reports have shown that physical and psychological indicators of the quality of life of kidney donors, compared to the general population, are maintained or even increased after donation.

Pregnancy after donation

Risks of pregnancy after donation should be explained well to female donors of child-bearing age or those who wish to give birth.

\section{Bibliography}

1. Ibrahim HN, et al. N Engl J Med. 2009;360:459-69. (Level 4)

2. Segev DL, et al. JAMA. 2010;303:959-66. (Level 4)

3. Okamoto M, et al. Transplantation. 2009;87:419-23. (Level 4)

4. Berger JC, et al. Clin J Am Soc Nephrol. 2011;6:2887-93. (Level 4)

5. Dols LF, et al. Am J Transplant. 2011;11:737-42. (Level 4)

6. Kido R, et al. Am J Transplant. 2009;9:2514-9. (Level 4)

7. Kido R, et al. Clin Exp Nephrol. 2010;14:356-62. (Level 4)

8. Garg AX, et al. Kidney Int. 2006;70:1801-10. (Level 1)

9. Yazawa M, et al. Clin Exp Nephrol. 2011;15:514-21. (Level 5)

10. Kido R, et al. Am J Transplant. 2010;10:1597-604. (Level 4)

11. Garg AX, et al. Transplantation. 2008;86:399-406. (Level 4)

12. Boudville N, et al. Ann Intern Med. 2006;145:185-96. (Level 1)

13. Mjøen G, et al. Am J Transplant. 2011;11:1315-9. (Level 4)

14. Clemens K, et al. Am J Transplant. 2011;11:463-9. (Level 4)

15. Ibrahim HN, et al. Am J Transplant. 2009;9:825-34. (Level 4)

16. Reisaeter AV, et al. Am J Transplant. 2009;9:820-4. (Level 4)

\section{Chapter 20: CKD care for the elderly}

CQ1 Is an evaluation for uroepithelial malignancy recommended for elderly patients with microscopic hematuria?

A higher chance of malignancy warrants medical checks with techniques such as ultrasonography, cystoscopy and urine cytology in elderly patients with microscopic hematuria.

In adults with asymptomatic gross or microscopic hematuria in the absence of proteinuria, the incidence of uroepithelial malignancy can be determined and has been found to increase with aging. Accordingly, asymptomatic hematuria in individuals 40 years of age or older is associated with an increased possibility of uroepithelial malignancy.

Although the likelihood of finding uroepithelial malignancy is higher in patients with macroscopic hematuria, asymptomatic hematuria, whether gross or microscopic, warrants evaluation. Ultrasonography, cystoscopy and urine cytology are of diagnostic value. According to recent research on patients with microscopic hematuria, the probability of undiagnosed malignant disease was less than $1 \%$. Patients who yield negative results in complete evaluations for asymptomatic microscopic hematuria have a low probability of subsequently developing uroepithelial malignancy. When hematuria is diagnosed for the first time in elderly patients, a further examination including diagnostic imaging should be performed to check for the occurrence of a urinary tract abnormality. If there are no abnormalities, no further examination is required, but an annual health check-up is recommended.

\section{Bibliography}

1. Mariani AJ, et al. J Urol. 1989;141:350-5. (Level 4)

2. Jung H, et al. J Urol. 2011;185:1698-703. (Level 4)

3. Badalament RA, et al. Cancer. 1987;60:1423-7. (Level 4)

4. Murakami S, et al. J Urol. 1990;144:99-101. (Level 4)

5. Edwards TJ, et al. BJU Int. 2011;107:247-52. (Level 4)

6. Cauberg EC, et al. J Endourol. 2011;25:1733-40. (Level 4)

7. Madeb R, et al. Urology. 2010;75:20-5. (Level 4) 
CQ2 Is percutaneous renal biopsy recommended for elderly patients with CKD?

We recommend use of renal biopsy for elderly patients with CKD be determined by weighing the undesirable side effects against the possible outcomes after a biopsy-proven diagnosis.

Percutaneous renal biopsy is a useful diagnostic aid that can guide decisions on therapeutic options for patients with CKD, even for the elderly. Since previous reports have shown that well-defined histological patterns do not always correspond to equally clear clinical pictures, particularly so in elderly patients with CKD, the diagnostic value of a renal biopsy for an elderly patient is obvious. Therefore, the use of renal biopsy for elderly patients with CKD should be determined by weighing the undesirable side effects against the possible outcomes after a biopsy-proven diagnosis.

\section{Bibliography}

1. Lindeman RD, et al. J Am Geriatr Soc. 1985;33:278-85. (Level 4)

2. Fehrman-Ekholm I, et al. Scand J Urol Nephrol. 2004;38:73-7. (Level 4)

3. Bleyer AJ, et al. Kidney Int. 2000;57:2072-9. (Level 4)

4. Ferro G, et al. Clin Nephrol. 2006;65:243-7. (Level 4)

CQ3 Should elderly patients with CKD be advised to quit smoking to prevent the progression of CKD?

We recommend that elderly patients with CKD quit smoking to prevent the progression of CKD. (Grade B)

Previous studies have reported that smoking is an independent risk factor for the development and progression of CKD, including diabetic nephropathy. This also has been demonstrated in elderly patients. Reducing or quitting smoking should be part of the therapy or regarded as a preventive measure for elderly patients with CKD.

Therefore, it is recommended that elderly patients with CKD quit smoking to prevent the progression of CKD to ESKD.

\section{Bibliography}

1. Tozawa M, et al. Kidney Int. 2002;62:956-62. (Level 4)

2. Yamagata K, et al. Kidney Int. 2007;71:159-66. (Level 4)

3. Haroun MK, et al. J Am Soc Nephrol. 2003;14:2934-41. (Level 4)

4. Chase HP, et al. JAMA. 1991;265:614-7. (Level 4)

5. Ikeda Y, et al. Diabetes Res Clin Pract. 1997;36:57-61. (Level 4)
6. Jones-Burton C, et al. Am J Nephrol. 2007;27:342-51. (Level 1)

7. Sawicki PT, et al. Diabetes Care. 1994;17:126-31. (Level 4)

8. Orth SR, et al. Nephrol Dial Transplant. 2005;20:2414-19. (Level 4)

9. Ejerblad E, et al. J Am Soc Nephrol. 2004;15:2178-85. (Level 4)

10. Stengel B, et al. Epidemiology. 2003;14:479-87. (Level 4)

11. Bleyer AJ, et al. Kidney Int. 2000;57:2072-9. (Level 4)

12. Gambaro G, et al. Diabetes Nutr Metab. 2001;14:337-42. (Level 4)

CQ4 Is vaccination recommended for elderly patients with CKD?

Since the rates of hospitalization for severe pneumonia or death are high among unvaccinated, highrisk persons such as elderly patients with CKD, we recommend that elderly patients with CKD receive influenza and pneumococcal vaccinations. (Grade C1)

Studies on the benefits of influenza vaccination among elderly persons with chronic lung disease, heart disease, and liver disease have shown that there are significant benefits associated with vaccination. Previous studies also demonstrated that the rates of hospitalization for pneumonia/ influenza or death were highest among unvaccinated, highrisk persons such as elderly patients with $\mathrm{CKD}$. Influenza vaccination reduces the frequency of secondary complications as well as the risk for influenza-related hospitalization and death among adults aged $\geq 66$ years with CKD.

Pneumococcal infections are a major source of pneumonia in adults, especially among older adults aged $\geq 66$ years with CKD. Pneumococcal conjugate vaccine is recommended for adults aged $\geq 65$ years with $\mathrm{CKD}$ or nephrotic syndrome as well as chronic heart disease, chronic lung disease, or diabetes.

\section{Bibliography}

1. Hak E, et al. Clin Infect Dis. 2002;35:370-7. (Level 4)

2. Collins AJ, et al. Am J Kidney Dis. 2008;51:S1-S320. (Level 4)

3. Snyder JJ, et al. J Am Soc Nephrol. 2009;20:1614-22. (Level 4)

CQ5 Is a low protein diet recommended for elderly patients with CKD to control the progression of CKD?

We suggest a low protein diet for elderly patients with CKD to slow the progression of CKD to ESKD. (Grade C1)

The recommended protein intake for elderly patients with CKD is $0.8 \mathrm{~g} / \mathrm{kg} / \mathrm{day}$. 
Previous studies suggested that dietary protein restriction can slow progression to ESKD in patients with CKD stage G3b or later. However, it is unclear whether a low protein diet is also recommended for elderly patients with CKD. Since most studies included adults aged $\geq 65$ years with CKD, a possible beneficial effect of a low protein diet in elderly patients with CKD cannot be denied. However, since some elderly patients with CKD are frail, the indication should be carefully determined. The recommended protein intake for elderly patients with CKD is $0.8 \mathrm{~g} / \mathrm{kg} /$ day, which is the same as that for adults with CKD.

\section{Bibliography}

1. Fouque D, et al. Nephrol Dial Transplant. 2000;15:1986-92. (Level 1)

2. Fouque D, et al. Cochrane Database Syst Rev. 2006;19(2):CD001892. (Level 1)

3. Rosman JB, et al. Lancet. 1984;2:1291-6. (Level 2)

4. Rosman JB, et al. Kidney Int. 1989;27(Suppl):S96-S102. (Level 3)

5. O'Hare AM, et al. J Am Soc Nephrol. 2007;18:2758-65. (Level 4)

6. Meloni C, et al. J Ren Nutr. 2002;12:96-101. (Level 3)

7. Meloni C, et al. J Ren Nutr. 2004;14:208-13. (Level 3)

8. Brunori G, et al. Am J Kidney Dis. 2007;49:569-80. (Level 2)

9. Menon V, et al. Am J Kidney Dis. 2009;53:208-17. (Level 3)

CQ6 Is salt restriction recommended to slow the progression of CKD in elderly patients with CKD?

We suggest a low-sodium diet for elderly hypertensive patients with CKD, since it is also effective for lowering blood pressure. (Grade $\mathrm{C} 1$ )

The target for salt intake is between $3 \mathrm{~g} /$ day and $6 \mathrm{~g} /$ day.

Studies performed in elderly people have revealed that dietary sodium intake probably has an impact on blood pressure as blood pressure is reduced in association with the restriction of sodium intake. Therefore, a low-sodium diet is likely to be effective for lowering the blood pressure of CKD patients and, therefore, also effective for slowing the progression of CKD, even in the elderly. The target salt intake recommended for elderly CKD patients is 3-6 g/ day, as is the case for non-elderly CKD patients. However, clinicians should be cautious about an excessive decline of blood pressure and hyponatremia due to a very low dietary sodium intake.

\section{Bibliography}

1. Luft FC, et al. Am J Hypertens. 1992;5:520-8. (Level 4)

2. Appel LJ, et al. Arch Intern Med. 2001;161:685-93. (Level 2)

3. Alam S, et al. J Hum Hypertens. 1999;13:367-74. (Level 1)
CQ7 Is antihypertensive therapy recommended to prevent the progression of CKD in elderly hypertensive patients with CKD?

In elderly hypertensive patients with CKD, we recommend antihypertensive therapy to slow the progression of renal impairment and to reduce the risk of CVD events. (Grade B)

For elderly hypertensive non-diabetic patients with CKD, we recommend the blood pressure be reduced gradually until the target of $<140 / 90 \mathrm{mmHg}$ is achieved. (Grade C1)

In elderly hypertensive non-diabetic patients with proteinuria (A2 or A3 categories of CKD) and elderly hypertensive non-diabetic CKD patients with a high risk of CVD, we recommend that blood pressure be further reduced gradually until the target of $<130 /$ $80 \mathrm{mmHg}$ is achieved, while carefully checking that there is no deterioration of kidney function and no ischemic symptoms of other organs. (Grade C1)

Excessive reduction of blood pressure should be avoided because it can compromise the survival benefit.

In elderly hypertensive diabetic patients with CKD, we recommend that blood pressure be reduced gradually towards the target of $<130 / 80 \mathrm{mmHg}$ while carefully checking that there is no deterioration of kidney function and no ischemic symptoms of other organs. (Grade C1)

For elderly hypertensive patients with CKD, we recommend that antihypertensive therapy be initiated with a CCB, diuretic, or RAS inhibitor as monotherapy. (Grade C1)

If the reduction of blood pressure is insufficient, then combined antihypertensive therapy should be attempted.

Results indicating the target blood pressure for CKD patients have been reported, but there has only been a limited number of studies that specifically enrolled elderly patients with CKD. Results of a few studies have suggested that a systolic blood pressure of $160 \mathrm{mmHg}$ or lower could be the target of antihypertensive therapy aimed at inhibiting the progression of CKD in elderly patients with CKD. The target blood pressure may be set at a higher level for elderly patients with CKD than for younger patients with $\mathrm{CKD}$, although there is insufficient evidence at present to support this. However, tighter blood pressure control is preferable for elderly CKD patients with diabetes or proteinuria, who are at high risk of progression to ESKD and occurrence of CVD, including cerebrovascular disease. 
Based on these considerations, the above blood pressure targets have been recommended for elderly hypertensive patients with CKD.

In elderly hypertensive patients with $\mathrm{CKD}$, great care should be taken to avoid excessive reduction of blood pressure. Some studies of elderly CKD patients have demonstrated a J-curve relationship between the reduction of blood pressure and an increase in all-cause mortality and cerebrovascular morbidity. The lower limit of the target blood pressure range should be set individually for each patient according to his/her general condition, because it is currently difficult to establish the level in an empirical manner.

It has been reported that CCBs slow the progression of CKD in elderly patients with CKD. In addition, the efficacy of diuretics and RAS inhibitors in reducing the incidence of CVD in elderly patients with CKD is supported by accumulating evidence. Therefore, these antihypertensive agents have been recommended as first-line drugs.

\section{Bibliography}

1. Suzuki H, et al. Clin Exp Hypertens. 2001;23:189-201. (Level 4)

2. Beckett NS, et al. N Engl J Med. 2008;358:1887-98. (Level 2)

3. Fagard RH, et al. Arch Intern Med. 2007;167:1884-91. (Level 4)

4. Gueyffier F, et al. Lancet. 1999;353:793-6. (Level 4)

5. Staessen JA, et al. Lancet. 2000;355:865-72. (Level 1)

6. Collaborative Research Group. JAMA. 1991;265:3255-64. (Level 2)

7. Pahor M, et al. Arch Intern Med. 1998;158:1340-5. (Level 2)

8. Sesso R, et al. Nephrology (Carlton). 2008;13:99-103. (Level 4)

9. Young JH, et al. J Am Soc Nephrol. 2002;13:2776-82. (Level 4)

10. Okada T, et al. Hypertens Res. 2009;32:1123-9. (Level 4)

11. Agarwal R. Clin J Am Soc Nephrol. 2009;4:830-7. (Level 4)

12. Hayashi K, et al. Hypertens Res. 2010;33:1211-20. (Level 4)

13. Boutitie F, et al. Ann Intern Med. 2002;136:438-48. (Level 1)

14. Weiner DE, et al. J Am Soc Nephrol. 2007;18:960-6. (Level 4)

15. Denardo SJ, et al. Am J Med. 2010;123:719-26. (Level 4)

16. Somes GW, et al. Arch Intern Med. 1999;159:2004-9. (Level 4)

17. Kostis JB, et al. JAMA. 1997;278:212-6. (Level 4)
18. Meesserli FH, et al. JAMA. 1998;279:1903-7. (Level 1)

19. Dahlof B, et al. Lancet. 2002;359:995-1003. (Level 2)

20. Frances CD, et al. Arch Intern Med. 2000;160:2645-50. (Level 4)

CQ8 Is the target hemoglobin level of 11-13 g/dL recommended for the management of anemia in elderly patients with CKD?

In elderly patients with CKD, we suggest that ESA therapy be initiated for anemia if the hemoglobin level falls below $10 \mathrm{~g} / \mathrm{dL}$. (Grade $\mathrm{C} 1$ )

We recommend not to continue ESA therapy to maintain a $\mathrm{Hb}$ level of $\geq 13 \mathrm{~g} / \mathrm{dL}$, because it may increase the risk of CVD events. (Grade D)

Do not administer high-dose ESA therapy with the aim of improving ESA-resistant anemia in elderly patients with CKD, but instead investigate the mechanism of ESA resistance.

Based on the results from two RCTs (the CHOIR study and the TREAT study) on the management of CKD-associated anemia and improvement of QOL in elderly patients with CKD, the criterion for initiation of treatment is defined as an $\mathrm{Hb}$ level that is lower than $10 \mathrm{~g} / \mathrm{dL}$, which is also a recommended criterion for the treatment of anemia in younger patients with CKD. In addition, it is recommended that the $\mathrm{Hb}$ level should not be maintained at $13 \mathrm{~g} /$ $\mathrm{dL}$ or higher. Furthermore, in ESA-resistant elderly patients with CKD, caution should be exercised against using high-dose ESA therapy. Instead, it is recommended that the cause of resistance to ESA should be investigated.

\section{Bibliography}

1. Singh AK, et al. N Engl J Med. 2006;355:2085-98. (Level 2)

2. Szczech LA, et al. Kidney Int. 2008;74:791-8. (Level 4)

3. Pfeffer MA, et al. N Engl J Med. 2009;361:2019-32. (Level 2)

4. Solomon SD, et al. N Engl J Med. 2010;363:1146-55. (Level 4) 
CQ9 Is the target $\mathrm{HbA}_{1 \mathrm{c}}$ of $<6.9 \%$ recommended for glycemic control in diabetic elderly patients with CKD?

Since available evidence is insufficient to establish the efficacy of tight glycemic control in elderly diabetic patients with $\mathrm{CKD}$, we suggest the target $\mathrm{HbA}_{1 \mathrm{c}}<8.2 \%$. (Grade C1)

Elderly diabetic patients with CKD are at high risk of developing hypoglycemia and are often unaware of its signs. Therefore, glycemic control should be implemented with great care.

There has been a limited number of studies investigating the target $\mathrm{HbA}_{1 \mathrm{c}}$ in elderly diabetic patients with CKD. Tanaka et al. reported that an $\mathrm{HbA}_{1 \mathrm{c}}$ level $<8.2 \%$ is the preferred target in these patients. After consideration of other guidelines, glycemic control targeting an $\mathrm{HbA}_{1 \mathrm{c}}$ level $<8.2 \%$ is recommended for elderly diabetic patients with CKD.

\section{Bibliography}

1. Tanaka Y, et al. Diabetes Care. 1998;21:116-20. (Level 4)

2. Burge MR, et al. JAMA. 1998;279:137-43. (Level 2)

3. Ben-Ami H, et al. Arch Intern Med. 1999;159:281-4. (Level 5)

4. Murata GH, et al. Diabetes Res Clin Pract. 2004;65:61-7. (Level 4)

CQ10 Is statin therapy recommended for preventing the progression of renal impairment in elderly CKD patients with dyslipidemia?

In elderly CKD patients with dyslipidemia, we recommend treatment with statins since it has the potential to prevent the progression of CKD and can reduce the risk of CVD events. (Grade $\mathrm{C} 1$ )

We suggest LDL-C $<120 \mathrm{mg} / \mathrm{dL}$ or non-HDL$\mathrm{C}<150 \mathrm{mg} / \mathrm{dL}$ as the target range for control of hyperlipidemia in elderly patients with CKD. (Grade C1)

There has only been a limited number of studies assessing the efficacy of statins for preventing the progression of renal impairment, especially in elderly CKD patients with dyslipidemia. A meta-analysis conducted by Vidt et al. revealed short-term efficacy of rosuvastatin for improving renal function, but the long-term efficacy of statin remains to be explored.

Therefore, statin therapy is recommended for elderly CKD patients with dyslipidemia since it may prevent the progression of renal impairment and can also reduce the risk of CVD events. A target lipid level of $<120 \mathrm{mg} / \mathrm{dL}$ for
LDL-C or $<150 \mathrm{mg} / \mathrm{dL}$ for non-HDL-C is recommended for elderly patients with CKD as is the case for younger patients with CKD.

\section{Bibliography}

1. Vidt DG, et al. Am J Cardiol. 2006;97:1602-6. (Level 1)

2. Barigent C, et al. Lancet. 2011;25:2181-92. (Level 2)

CQ11 Is weight control recommended for obese elderly patients with CKD to slow the progression of CKD ?

We recommend weight control since it can slow the progression of CKD and improve exercise capacity for obese elderly patients with $\mathrm{CKD}$, with the proviso that excessive dieting and exercise should be avoided. (Grade C1)

Obesity is recognized increasingly as a major risk factor for the progression of CKD. Several studies have reported that weight control can slow the progression of CKD. However, whether or not obesity is a risk factor for elderly patients with CKD has not yet been evaluated sufficiently.

An epidemiologic study has reported that elderly patients with metabolic syndrome had a higher cumulative incidence and relative risk of CKD. On the other hand, other studies have suggested that metabolic syndrome is a significant determinant of CKD in men under 60 years of age, but not for older patients. However, based on all of these results, weight control is recommended for obese elderly patients with CKD, but excessive dieting and exercise should be avoided.

\section{Bibliography}

1. Janssen I, et al. Obes Rev. 2007;8:41-59. (Level 1)

2. Elsayed EF, et al. Am J Kidney Dis. 2008;52:49-57. (Level 4)

3. Chou CY, et al. Intern Med J. 2008;38:402-6. (Level 4)

4. Ninomiya T, et al. Am J Kidney Dis. 2006;48:383-91. (Level 4)

5. Tanaka H, et al. Kidney Int. 2006;69:369-74. (Level 4)

6. Tokashiki K, et al. Clin Exp Nephrol. 2009;13:55-60. (Level 4)

7. Leehey DJ, et al. Cardiovasc Diabetol. 2009;8:62. (Level 2)

8. Cook SA, et al. Nephrol Dial Transplant. 2008;23:263-8. (Level 4)

9. MacLaughlin HL, et al. Am J Kidney Dis. 2010;55:69-76. (Level 3)

CQ12 Is the administration of bisphosphonates recommended for elderly patients with CKD for the prevention and treatment of osteoporosis?

We recommend administration of bisphosphonates for elderly patients with CKD to decrease the incidence of bone fractures. (Grade B) 
There is a significant association between hip bone fracture and moderate to severe degrees of CKD. According to recent research, alendronate and risedronate are safe and effective for increasing bone matrix density and decreasing bone fractures in CKD patients, particularly female patients with severely reduced renal function. For elderly patients with CKD, we recommend bisphosphonate for the prevention and treatment of osteoporosis. Therapy using bisphosphonates against osteoporosis should be undertaken carefully to avoid undesirable side effects, such as jaw necrosis.

\section{Bibliography}

1. Nickolas TL, et al. J Am Soc Nephrol. 2006;17:3223-32. (Level 4)

2. Jamal SA, et al. J Bone Miner Res. 2007;22:503-8. (Level 2)

3. Miller PD, et al. J Bone Miner Res. 2005;20:2105-15. (Level 1)

4. Boonen S, et al. Kidney Int. 2008;74:641-8. (Level 2)

CQ13 Is immunosuppressive therapy combined with corticosteroid recommended for elderly patients with idiopathic nephrotic syndrome?

We recommend immunosuppressive therapy combined with corticosteroid for elderly patients with steroid-resistant idiopathic membranous nephropathy (Grade B) or FSGS (Grade C1) to induce remission. Immunosuppressive therapy for elderly patients should be determined carefully since the elderly patients are particularly prone to the adverse effects and infectious complications of immunosuppression. Conservative therapy with RAS inhibitors or diuretics may be preferable for elderly patients when they are highly immunocompromised or complicated.

A meta-analysis showed that treatment with immunosuppressives combined with corticosteroid increased the remission rate and was safe. In contrast, one observational study from Japan reported that corticosteroid monotherapy could induce remission in patients with idiopathic membranous nephropathy as effectively as therapy combined with the administration of immunosuppressives. Therefore, in Japan, treatment with immunosuppressives combined with corticosteroid has been recommended to induce remission in patients with steroid-resistant idiopathic membranous nephropathy. A retrospective cohort study reviewed the outcomes after at least 1 year of treatment with chlorambucil combined with corticosteroid in 41 patients with membranous nephropathy who were older than 65 years at onset. The study concluded that such therapy was effective for inducing remission and safe for elderly patients with membranous nephropathy.
For the treatment of steroid-resistant cases with FSGS, a previous RCT demonstrated that immunosuppressives combined with low-dose corticosteroid was effective. Another cohort study reported that corticosteroid monotherapy was effective for inducing remission in patients aged $\geq 66$ years with FSGS.

Considering these results, the administration of immunosuppressives combined with corticosteroid is recommended for elderly patients with steroid-resistant idiopathic membranous nephropathy in order to induce remission. For the treatment of FSGS, corticosteroid monotherapy is recommended as the first-line treatment and immunosuppressives combined with corticosteroid should be tried in elderly patients with steroid-resistant FSGS.

Immunosuppressive therapy for elderly patients should be undertaken carefully since the elderly are particularly prone to the adverse effects and infectious complications of immunosuppression. Conservative therapy with RAS inhibitors or diuretics is recommended when the patients are highly immunocompromised or complicated.

\section{Bibliography}

1. Perna A, et al. Am J Kidney Dis. 2004;44:385-401. (Level 1)

2. Ponticelli C, et al. J Am Soc Nephrol. 1998;9:444-9:4. (Level 2)

3. Jha V, et al. J Am Soc Nephrol. 2007;18:1899-18:1. (Level 2)

4. Shiiki H, et al. Kidney Int. 2004;65:1400-7. (Level 4)

5. Passerini P, et al. Nephrol Dial Transplant. 1993;8:1321-5. (Level4)

6. Cattran DC, et al. Kidney Int. 1999;56:2220-6. (Level 2)

7. Nagai R, et al. Clin Nephrol. 1994;42:18-21. (Level 4)

CQ14 Is treatment with corticosteroid recommended for elderly patients with IgAN to suppress the progression of renal dysfunction?

We recommend treatment with corticosteroid for elderly patients with IgAN to slow the progression of renal dysfunction. (Grade $\mathrm{C} 1$ )

For elderly patients with IgAN, conservative therapy with RAS inhibitors or anti-platelets could be selected from the aspects of possible complications and life expectancy.

The current cumulative evidence suggests that corticosteroid has significant effects on protecting renal function and reducing proteinuria in patients with IgAN, but whether or not it is also effective for elderly patients remains unclear. When the histological diagnosis of IgAN was established, poor prognosis indices, such as systolic high blood pressure, severe proteinuria, decreased Ccr, and severely impaired histology, were more commonly present in elderly patients aged over 50 years than those under 50 years. Therefore, treatment with corticosteroid is recommended to slow the progression of renal dysfunction in elderly patients with IgAN. 


\section{Bibliography}

1. Cheng J, et al. Am J Nephrol. 2009;30:315-22. (Level 4)

2. Zhou YH, et al. PLoS One. 2011;6:e18788. (Level 4)

3. Frimat L, et al. Nephrol Dial Transplant. 1996;11:1043-7. (Level 4)

CQ15 Is the initiation of maintenance dialysis in accordance with the standard criteria recommended for elderly patients with CKD?

For patients with stage G5 CKD, we recommend that appropriate timing of the initiation of dialysis be determined in accordance with the standard criteria. (Grade C1) For high-risk patients such as elderly patients with CKD, early initiation of dialysis may be desirable to avoid complications and to improve the QOL.

Current recommendations for initiating dialysis therapy are based on the level of residual kidney function and clinical symptoms due to uremia. Effects of the early initiation of dialysis remain unclear. Since previous studies reported that patients who were initiated on dialysis therapy at greater GFRs were at an increased risk of death that was not fully explained by comorbidity, early initiation of dialysis is not recommended. There is no evidence showing the beneficial effects of early initiation of dialysis in elderly patients. Therefore, for patients with stage G5 $\mathrm{CKD}$, the appropriate time to initiate dialysis should be determined in accordance with the standard criteria. For high-risk patients such as the elderly with CKD, early initiation of dialysis may be desirable to avoid complications and to improve the QOL.

\section{Bibliography}

1. Biesenbach G, et al. Ren Fail. 2002;24:197-205. (Level 4)

2. Bradbury BD, et al. Clin J Am Soc Nephrol. 2007;2:89-99. (Level 4)

3. Kazmi WH, et al. Am J Kidney Dis. 2005;46:887-96. (Level 4)

4. Wright S, et al. Clin J Am Soc Nephrol. 2010;5:1828-35. (Level 4)

5. Harris A, et al. Am J Kidney Dis. 2011;57:707-15. (Level 2)

6. Cooper BA, et al. N Engl J Med. 2010;363:609-19. (Level 2)
CQ16 Is kidney transplantation recommended for the treatment of ESKD in elderly patients with CKD?

We recommend kidney transplantation for the treatment of ESKD in elderly patients with CKD since their graft survival rate is similar to that in younger patients and transplantation may improve the prognosis. (Grade B)

It was reported that the graft survival rate and prognosis of elderly recipients were inferior to those of younger recipients. However, death with a functioning graft (DWFG) contributed to the lower graft survival rate in elderly recipients, rather than graft failure. Most analyses that used DWFG as an endpoint have revealed that the graft survival rate is similar in elderly recipients to that in younger recipients, and some of these analyses have even shown that the graft survival rate is higher in elderly recipients than in younger recipients.

A study of elderly dialysis patients on a transplant waiting list compared those who received a kidney transplant with those who remained on dialysis. The results revealed that kidney transplant recipients had a worse short-term survival rate, but showed significantly superior long-term survival. For the management of ESRD in elderly patients, there is no evidence suggesting that an age limit be set for receiving a kidney transplant. Therefore, as with younger CKD patients, the risk vs. benefit balance should be considered for each patient and kidney transplantation should be selected if so indicated.

\section{Bibliography}

1. Eufrásio P, et al. Transplant Proc. 2011;43:117-9. (Level 4)

2. Oniscu GC, et al. Am J Transplant. 2004;4:2067-74. (Level 4)

3. Huang E, et al. Transplantation. 2010;90:974-9. (Level 4)

4. Chuang FP, et al. Transplant Proc. 2008;40:2299-302. (Level 4)

5. Tesi RJ, et al. Lancet. 1994;343:461-4. (Level 4)

6. Roodnat JI, et al. Transplantation. 1999;67:576-80. (Level 4)

7. Hernández D, et al. Transplantation. 2005;79:337-43. (Level 4)

8. Ojo AO, et al. Kidney Int. 2000;57:307-13. (Level 4)

9. Gill J, et al. Am J Kidney Dis. 2008;52:541-52. (Level 4) 
10. Kasiske BL, et al. Am J Transplant. 2004;4:905-13. (Level 4)

11. Mohamed Ali AA, et al. Int Urol Nephrol. 2011;43:265-71. (Level 4)

12. Heldal K, et al. Nephrol Dial Transplant. 2010;25:1680-7. (Level 4)

13. Martín Navarro J, et al. Transplant Proc. 2009;41:2376-8. (Level 4)

CQ17 Is kidney donation from an elderly person disadvantageous for the functional outcome of the recipient after receiving a kidney transplant?

Transplantation of kidneys from elderly donors may be inferior to using grafts from younger donors in terms of post-transplantation outcomes. Nevertheless, the use of kidneys from elderly donors should not be ruled out because of a shortage of donors.

There have been a number of reports that kidney transplantation from elderly donors is inferior to transplantation from younger donors with respect to posttransplantation outcomes (graft survival rate and patient survival rate).

However, in a study of living-donor kidney transplantation to patients aged $\geq 60$ years, and which employed the OPTN/UNOS database, multivariate analysis revealed that both the graft survival rate and patient survival were comparable between living donors aged over 55 years and those aged 55 years or younger.

There is a shortage of donors, hence kidney transplantation from elderly donors should not be ruled out and its appropriateness should be considered for each patient individually. Elderly living donors should be followed up with great care after the kidney graft has been harvested.

\section{Bibliography}

1. Rizzari MD, et al. Transplantation. 2011;92:70-5. (Level 4)

2. Gentil MA, et al. Transplant Proc. 2010;42:3130-3. (Level 4)

3. Gill J, et al. Am J Kidney Dis. 2008;52:541-52. (Level 4)

4. Young A, et al. Am J Transplant. 2011;11:743-50. (Level 4)

5. Galeano C, et al. Transplant Proc. 2010;42:3935-7. (Level 4)

6. Cassini MF, et al. Transplant Proc. 2010;42:417-20. (Level 4)

7. Gavela E, et al. Transplant Proc. 2009;41:2047-9. (Level 4)

8. Fehrman-Ekholm I, et al. Transplantation. 2006;82:1646-8. (Level 4)

9. Najarian JS, et al. Lancet. 1992;340:807-10. (Level 4)

10. Gossmann J, et al. Am J Transplant. 2005;5:2417-24. (Level 4)

11. Saran R, et al. Nephrol Dial Transplant. 1997;12:1615-21. (Level 4)
CQ18 Is the use of iodinated contrast medium recommended for elderly patients with $\mathrm{CKD}$ ?

In elderly CKD patients, the incidence of contrastinduced nephropathy is high. Therefore, we recommend that the appropriateness of performing contrastenhanced imaging in elderly patients with CKD be examined carefully.

If the need for contrast-enhanced imaging is thought to outweigh the risks of contrast-induced nephropathy (CIN) in elderly patients with CKD, the minimum dose of contrast medium should be used after providing the patient with an adequate explanation about CIN, and ensuring adequate prophylactic measures (such as hydration) to avoid CIN before and after imaging.

In many reports, aging is referred to as an independent risk factor for CIN. A systematic review published in 2007 lists the following as classic risk factors for CIN: preexisting renal insufficiency, diabetes mellitus, advanced age, nephrotoxic substances, dehydration, use of high doses of contrast medium, ionic high-osmolar contrast media, and congestive heart failure.

Based on the above, iodinated contrast media should not be used in elderly patients with CKD whenever possible, because of the high incidence of CIN in this patient group. If the need for contrast-enhanced imaging is thought to outweigh the risks in elderly patients with $\mathrm{CKD}$, the contrast medium should be administered at the minimum dose after providing the patient with an adequate explanation about $\mathrm{CIN}$, and ensuring adequate prophylactic measures (such as hydration) to avoid CIN before and after imaging.

\section{Bibliography}

1. Chong E, et al. Ann Acad Med Singapore. 2010;39:374-80. (Level 4)

2. Mehran R, et al. J Am Coll Cardiol. 2004;44:1393-9. (Level 4)

3. Toprak O. J Urol. 2007;178:2277-83. (Level 1)

CQ19 Are COX-2-selective NSAIDs recommended as anti-inflammatory/analgesic medications for elderly patients with CKD?

We recommend that use of NSAIDs be minimized in elderly patients with CKD, regardless of whether these drugs are selective or non-selective, since, as with non-selective NSAIDs, COX-2-selective NSAIDs have the potential to cause progression of renal impairment. (Grade C2) 
A few studies have compared the effects of COX-2selective NSAIDs and non-selective NSAIDs on renal function in elderly patients with CKD, and none of these studies has demonstrated any advantage of COX-2-selective NSAIDs. Therefore, minimizing the use of NSAIDs is recommended in elderly patients with CKD, irrespective of whether these drugs are COX-2-selective or non-selective.

\section{Bibliography}

1. Swan SK, et al. Ann Intern Med. 2000;133:1-9. (Level 2)

2. Gooch K, et al. Am J Med. 2007;120:280.e1-7. (Level 4)

\section{Chapter 21: Drug administration in CKD}

CQ1 Does contrast medium affect the progression of CKD?

Contrast medium affects the progression of CKD through the onset of contrast-induced nephropathy (CIN).

CKD patients at stage G3b or greater $\left(\right.$ GFR $\left.<45 \mathrm{~mL} / \mathrm{min} / 1.73 \mathrm{~m}^{2}\right)$ are at an increased risk of CIN after contrast-enhanced CT.

CKD patients at stage G3a or greater (GFR $<60 \mathrm{~mL} /$ $\min / 1.73 \mathrm{~m}^{2}$ ) are at an increased risk of CIN after CAG.

CIN is generally defined as increases equal to $0.5 \mathrm{mg} / \mathrm{dL}$ or higher or increases equal to $25 \%$ or higher in creatinine level at $72 \mathrm{~h}$ after the administration of iodinated contrast medium. To avoid the onset of CIN, it is important to predict the risk before the administration of contrast medium.

In a cohort study of 1,144 patients receiving CAG with non-ionic contrast medium, baseline renal impairment was the only confirmed predictor of CIN, and there was an exponential increase in the risk of CIN if the baseline creatinine level was $1.20 \mathrm{mg} / \mathrm{dL}$ or higher. CIN developed in 381 of 1,980 patients $(19.2 \%$ ) with CKD (eGFR $<60 \mathrm{~mL} / \mathrm{min} / 1.73 \mathrm{~m}^{2}$ ) and in 688 of 5,250 patients $(13.1 \%)$ without CKD after PCI.

After undergoing contrast-enhanced $\mathrm{CT}$ in an outpatient setting, Weisbord et al. reported that patients with an eGFR level of less than $45 \mathrm{ml} / \mathrm{min} / 1.73 \mathrm{~m}^{2}$ were at a higher risk of CIN. Kim et al. reported that the incidence of CIN was $0.0,2.9$, and $12.1 \%$ in patients with an eGFR of $45-59$, $30-44$, and $<30 \mathrm{~mL} / \mathrm{min} / 1.73 \mathrm{~m}^{2}$, respectively.

\section{Bibliography}

1. Lameire N, et al. Am J Cardiol. 2006;98(suppl):21K-6K. (Level 6)
2. Davidson CJ, et al. Ann Intern Med. 1989;110:119-24. (Level 4)

3. Dangas G, et al. Am J Cardiol. 2005;95:13-9. (Level 4)

4. Rihal CS, et al. Circulation. 2002;105:2259-64. (Level 4)

5. Weisbord SD, et al. Clin J Am Soc Nephrol. 2008;3:1274-81. (Level 4)

6. Kim SM, et al. Am J Kidney Dis. 2010;55:1018-25. (Level 3)

CQ2 Is fluid therapy recommended for the prevention of CIN?

Since an intravenous infusion of isotonic saline prevents CIN, we recommend infusions of isotonic saline before and after the administration of contrast medium. (Grade A)

We suggest intravenous infusion, especially shorttime infusion of sodium bicarbonate solution, because it prevents CIN and the effect may be superior to that of the infusion of isotonic saline. (Grade C1)

At first, a $0.45 \%$ isotonic sodium chloride solution was used for the prevention of CIN. RCTs that investigated the importance of osmotic pressure in fluid therapy were conducted, and the findings from analysis of 1,620 patients demonstrated that the effect of isotonic saline was superior to that of $0.45 \%$ saline. Therefore the administration of isotonic saline significantly reduced the incidence of CIN. In many studies, administration of isotonic saline has been carried out at the rate of $1 \mathrm{~mL} / \mathrm{kg} / \mathrm{h}, 6-12 \mathrm{~h}$ before and after the examination. Based on these results, we recommend the administration of isotonic saline for the prevention of contrast-induced nephropathy.

On the other hand, 4 RCTs compared the effect of rapid infusion $(3 \mathrm{~mL} / \mathrm{kg} / \mathrm{h})$ of sodium bicarbonate solution $(150 \mathrm{mEq} / \mathrm{L}) 1 \mathrm{~h}$ before examination, followed by that administration at $1 \mathrm{~mL} / \mathrm{kg} / \mathrm{h}$ for 6 to $12 \mathrm{~h}$. These RCTs, including the PREVENT study reported from Korea, demonstrated that the incidence of CIN was equal to or significantly lower in the sodium bicarbonate solution group compared to the isotonic saline group. These results suggest that sodium bicarbonate solution may reduce the incidence of CIN more effectively than isotonic saline in cases when the treatment time is limited. However, no study has reported that sodium bicarbonate solution reduced the incidence of dialysis therapy or mortality.

\section{Bibliography}

1. Eisenberg RL, et al. Am J Med. 1980;68:43-6. (Level 4)

2. Eisenberg RL, et al. Am J Roentgenol. 1981;136:859-61. (Level 4)

3. Mueller C, et al. Arch Intern Med. 2002;162:329-36. (Level 2) 
4. Trivedi HS, et al. Nephron Clin Pract. 2003;93:C29-34. (Level 2)

5. Zoungas S, et al. Ann Intern Med. 2009;151:631-8. (Level 1)

6. Meier P, et al. BMC Med. 2009;7:23. (Level 1)

7. Kanbay M, et al. Int Urol Nephrol. 2009;41:617-27. (Level 1)

8. Hogan SE, et al. Am Heart J. 2008;156:414-21. (Level 1)

9. Joannidis M, et al. Wien Klin Wochenschr. 2008;120:742-8. (Level 1)

10. Navaneethan SD, et al. Am J Kidney Dis. 2009;53:617-27. (Level 1)

11. Trivedi H, et al. Clin Nephrol. 2010;74:288-96. (Level 1)

12. Ueda H, et al. Am J Cardiol. 2011;107:1163-7. (Level 2)

13. Tamura A, et al. Am J Cardiol. 2009;104:921-5. (Level 2)

14. Morihito M, et al. Am J Cardiol. 2011;107:1604-8. (Level 2)

15. Briguori C, et al. Circulation. 2007;115:1211-7. (Level 2)

16. Maioli M, et al. J Am Coll Cardiol. 2008;52:599-604. (Level 2)

17. Shavit L, et al. J Interv Cardiol. 2009;22:556-63. (Level 2)

18. Lee SW, et al. Am J Cardiol. 2011;107:1447-52. (Level 2)

19. Vasheghani-Farahani A, et al. Am J Kidney Dis. 2009;54:610-8. (Level 2)

20. Vasheghani-Farahani A, et al. J Nephrol. 2010;23:216-23. (Level 2)

CQ3 Is blood purification therapy recommended for the prevention of CIN?

We do not recommend hemodialysis after the administration of contrast media, because it is not effective for preventing the onset of CIN. (Grade D)

Since contrast medium can be removed by hemodialysis (HD), there have been several reports of studies that tested the effectiveness of blood purification therapy for preventing the development of CIN. However, most studies were not able to demonstrate the preventive effect. Vogt et al. performed an RCT to test whether CIN can be avoided by conducting prophylactic hemodialysis immediately after CAG, PTA, and PTRA, and found that it did not diminish the rate of complications, including the development of CIN, the development of CVD, and death, during a 6-day period after administration of contrast medium. Moreover, Reinecke et al. reported that the incidence of CIN from 48 to $72 \mathrm{~h}$ after CAG was higher in patients receiving HD.

Lee et al. examined the effect of HD on preventing the development of CIN after CAG. Ccr of patients receiving HD decreased more than in those without HD $(0.4 \pm 0.9$ vs. $2.2 \pm 2.8 \mathrm{ml} / \mathrm{min} / 1.73 \mathrm{~m}^{2}$ ). Additionally, the number of patients requiring temporary dialysis was lower in the dialysis group. However, these results need to be interpreted cautiously, because this was a single study and there have been no other studies with similar results; moreover, this study included relatively advanced CKD patients with a mean creatinine level of $4.9 \mathrm{mg} / \mathrm{dL}$.

\section{Bibliography}

1. Vogt B, et al. Am J Med. 2001;111:692-8. (Level 2)

2. Sterner G, et al. Scand J Urol Nephrol. 2000;34:323-6. (Level 2)

3. Lehnert T, et al. Nephrol Dial Transplant. 1998;13:358-62. (Level 2)

4. Frank H, et al. Clin Nephrol. 2003;60:176-82. (Level 2)

5. Reinecke H, et al. Clin Res Cardiol. 2007; 96:130-9. (Level 2)

6. Shiragami K, et al. Circ J. 2008;72:427-33. (Level 2)

7. Lee PT, et al. J Am Coll Cardiol. 2007;50:1015-20. (Level 2)

8. Marenzi G, et al. N Engl J Med. 2003;349:1333-40. (Level 2)

9. Marenzi G, et al. Am J Med. 2006;119:155-62. (Level 2)

10. Song K, et al. Am J Nephrol. 2010;32:497-504. (Level 1)

CQ4 Do NSAIDs affect the progression of CKD?

In patients with CKD, all NSAIDs carry the risk of causing further deterioration of kidney function. However, it is not known whether or not the renal deterioration by NSAIDs affects the long-term prognosis of CKD.

Some reports have shown significant relationships between renal disorder and COX non-selective NSAIDs, or COX-2 selective NSAIDs that have been introduced to reduce renal disorder or gastrointestinal mucosal disorder, while other reports do not, and currently there is no consensus on the safety of these drugs. In the first edition of the CKD guideline, we commented that the use of NSAIDs should be minimal, because all NSAIDs carry the risk of kidney disorder. Subsequently, there has been no evidence to establish the safety of these drugs. A recent report from the United States showed that many CKD patients were potential users of NSAIDs, including commercially available drugs, and the awareness of CKD did not affect the amounts of NSAIDs consumed. It is important to enlighten patients with CKD regarding the use of NSAIDs.

\section{Bibliography}

1. Perneger TV, et al. N Engl J Med. 1994;331:1675-9. (Level 4)

2. Rexrode KM, et al. JAMA. 2001;286:315-21. (Level 4)

3. Fored CM, et al. N Engl J Med. 2001;345:1801-8. (Level 4)

4. Temple AR, et al. Clin Ther. 2006;28:222-35. (Level 2)

5. Evans M, et al. Nephrol Dial Transplant. 2009;24:1908-18. (Level 4)

6. Murray MD, et al. Am J Med Sci. 1995;310:188-97. (Level 2)

7. Whelton A, et al. Ann Intern Med. 1990;112:568-76. (Level 2)

8. Cook ME, et al. J Rheumatol. 1997;24:1137-44. (Level 2)

9. Gooch K, et al. Am J Med. 2007;120:280.e1-7. (Level 4)

10. Swan SK, et al. Ann Intern Med. 2000;133:1-9. (Level 2)

11. Whelton A, et al. Arch Intern Med. 2000;160:1465-70. (Level 2)

12. Whelton A, et al. Kidney Int. 2006;70:1495-502. (Level 2)

13. Plantinga L, et al. Ann Fam Med. 2011;9:423-30. (Level 4) 
CQ5 Is the carbonaceous oral adsorbent, AST-120, recommended for preventing the progression of CKD?

We recommend that the carbonaceous oral adsorbent, AST-120, be considered for patients with CKD, since AST-120 partially improves the markers of CKD progression, suggesting a potential effect of slowing the progression of CKD. (Grade C1)

Several studies have reported that AST-120 slowed the deterioration of the CKD markers derived from serum creatinine levels, however there have been no reports that AST-120 affected the incidence of end-points, such as mortality and the need for dialysis. Therefore, the administration of AST-120 is not strongly recommended, but can be taken into account, since it partially improves the markers of kidney function and has the potential effect of slowing the progression of $\mathrm{CKD}$

\section{Bibliography}

1. Akizawa T, et al. Am J Kidney Dis. 2009;54:459-67. (Level 2)

2. Nakamura T, et al. Metabolism. 2011;60:260-4. (Level 3)

3. Konishi K, et al. Diabetes Res Clin Pract. 2008;81:310-5. (Level 2)

4. Owada A, et al. Kidney Int 1997; 63(suppl):S188-90. (Level 2)

5. Shoji T, et al. Nephron Clin Pract. 2007;105:c99-107. (Level 2)

6. Ueda H, et al. Ther Apher Dial. 2007;11:189-95. (Level 4)

7. Schulman G, et al. Am J Kidney Dis. 2006;47:565-77. (Level 2)

8. Yorioka N, et al. J Nephrol. 2008;21:213-20. (Level 2)

9. Nakamura T, et al. Kidney Blood Press Res. 2004;27:121-6. (Level 2)

CQ6 Does the risk of nephrogenic systemic fibrosis (NSF) from MRI contrast medium containing gadolinium increase in patients with CKD?

As the CKD stage progresses, the risk of NSF by MRI contrast medium containing gadolinium increases. The risk of NSF by gadolinium is high in patients at CKD stage G4/G5 and with ESKD requiring dialysis therapy (CKD stage G5D).

Since NSF induced by gadolinium is found in patients with $\mathrm{CKD}$ stage $\mathrm{G} 3 \mathrm{a} / \mathrm{G} 3 \mathrm{~b}$, the necessity and risk of its use should be considered in deciding whether or not to use a gadolinium contrast medium.

The relationship between CKD stage G1/G2 and the incidence of NSF induced by gadolinium is not known.
By the year 2006, a series of cases had shown the relationship between the incidence of NSF and administration of gadolinium contrast medium. Subsequently, analyses have been carried out on the relationship between CKD stage, type or dose of gadolinium contrast medium and the incidence of NSF.

Patients with ESKD on maintenance dialysis therapy and patients before dialysis therapy at CKD stage G4/G5 with an eGFR of less than $30 \mathrm{~mL} / \mathrm{min} / 1.73 \mathrm{~m}^{2}$ are at increased risk of NSF and are considered to be a high risk group for NSF. Accordingly, the use of gadolinium contrast medium should be avoided in these advanced CKD patients at the time of MRI imaging. Some reports have shown that the risks of NSF were not high in patients at CKD stage G3a/G3b, with an eGFR in the range of $30 \mathrm{~mL} / \mathrm{min} / 1.73 \mathrm{~m}^{2}$ or more to less than $60 \mathrm{~mL} / \mathrm{min} / 1.73 \mathrm{~m}^{2}$, while other reports have shown NSF cases at these CKD stages. Therefore, necessity and risk should be carefully taken into consideration when deciding on the use of gadolinium contrast medium. Furthermore, if it is used, a minimal dose should be selected.

There is not enough evidence to suggest that the incidence of NSF is high in patients of CKD at stage G1/G2 with an eGFR of $60 \mathrm{~mL} / \mathrm{min} / 1.73 \mathrm{~m}^{2}$ or more.

\section{Bibliography}

1. Deo A, et al. Clin J Am Soc Nephrol. 2007; 2:264-7. (Level 4)

2. Rydahl C, et al. Invest Radiol. 2008;43:141-4. (Level 4)

3. Prince MR, et al. Radiology. 2008;248:807-16. (Level 4)

4. Agarwal R, et al. Nephrol Dial Transplant. 2009;24:856-63. (Level 4)

5. Chrysochou C, et al. Clin J Am Soc Nephrol. 2010;5:484-9. (Level 4)

6. Reilly RF. Clin J Am Soc Nephrol. 2008;3:747-51. (Level 4)

7. Perez-Rodriguez J, et al. Radiology. 2009;250:371-7. (Level 4)

8. Othersen JB, et al. Nephrol Dial Transplant. 2007;22:3179-85. (Level 4)

9. Sadowski EA, et al. Radiology. 2007;243:148-57. (Level 4) 